TRANSACTIONS OF THE

AMERICAN MATHEMATICAL SOCIETY

Volume 362, Number 5, May 2010, Pages 2723-2787

S 0002-9947(09)04981-2

Article electronically published on December 17, 2009

\title{
DECOMPOSITION NUMBERS FOR FINITE COXETER GROUPS AND GENERALISED NON-CROSSING PARTITIONS
}

\author{
C. KRATTENTHALER AND T. W. MÜLLER
}

\begin{abstract}
Given a finite irreducible Coxeter group $W$, a positive integer $d$, and types $T_{1}, T_{2}, \ldots, T_{d}$ (in the sense of the classification of finite Coxeter groups), we compute the number of decompositions $c=\sigma_{1} \sigma_{2} \cdots \sigma_{d}$ of a Coxeter element $c$ of $W$, such that $\sigma_{i}$ is a Coxeter element in a subgroup of type $T_{i}$ in $W, i=1,2, \ldots, d$, and such that the factorisation is "minimal" in the sense that the sum of the ranks of the $T_{i}$ 's, $i=1,2, \ldots, d$, equals the rank of $W$. For the exceptional types, these decomposition numbers have been computed by the first author in ["Topics in Discrete Mathematics," M. Klazar et al. (eds.), Springer-Verlag, Berlin, New York, 2006, pp. 93-126] and [Séminaire Lotharingien Combin. 54 (2006), Article B541]. The type $A_{n}$ decomposition numbers have been computed by Goulden and Jackson in [Europ. J. Combin. 13 (1992), 357-365], albeit using a somewhat different language. We explain how to extract the type $B_{n}$ decomposition numbers from results of Bóna, Bousquet, Labelle and Leroux [Adv. Appl. Math. 24 (2000), 22-56] on map enumeration. Our formula for the type $D_{n}$ decomposition numbers is new. These results are then used to determine, for a fixed positive integer $l$ and fixed integers $r_{1} \leq r_{2} \leq \cdots \leq r_{l}$, the number of multi-chains $\pi_{1} \leq \pi_{2} \leq \cdots \leq \pi_{l}$ in Armstrong's generalised non-crossing partitions poset, where the poset rank of $\pi_{i}$ equals $r_{i}$ and where the "block structure" of $\pi_{1}$ is prescribed. We demonstrate that this result implies all known enumerative results on ordinary and generalised non-crossing partitions via appropriate summations. Surprisingly, this result on multi-chain enumeration is new even for the original non-crossing partitions of Kreweras. Moreover, the result allows one to solve the problem of rank-selected chain enumeration in the type $D_{n}$ generalised non-crossing partitions poset, which, in turn, leads to a proof of Armstrong's $F=M$ Conjecture in type $D_{n}$, thus completing a computational proof of the $F=M$ Conjecture for all types. It also allows one to address another conjecture of Armstrong on maximal intervals containing a random multi-chain in the generalised non-crossing partitions poset.
\end{abstract}

\section{INTRODUCTION}

The introduction of non-crossing partitions for finite reflection groups (finite Coxeter groups) by Bessis [8] and Brady and Watt [15] marks the creation of a

Received by the editors June 30, 2008 and, in revised form, December 10, 2008.

2000 Mathematics Subject Classification. Primary 05E15; Secondary 05A05, 05A10, 05A15, 05A18, 06A07, 20F55, 33C05.

Key words and phrases. Root systems, reflection groups, Coxeter groups, generalised noncrossing partitions, annular non-crossing partitions, chain enumeration, Möbius function, $M$ triangle, generalised cluster complex, face numbers, $F$-triangle, $\mathrm{Chu}$-Vandermonde summation.

The first author's research was partially supported by the Austrian Science Foundation FWF, grant S9607-N13, in the framework of the National Research Network "Analytic Combinatorics and Probabilistic Number Theory".

(C)2009 American Mathematical Society 2723

Reverts to public domain 28 years from publication 
new, exciting subject of combinatorial theory, namely the study of these new combinatorial objects which possess numerous beautiful properties and seem to relate to several other objects of combinatorics and algebra, most notably to the cluster complex of Fomin and Zelevinsky [21] (cf. [2, 3, 4, 15, 8, 9, 14, 15, 16, 17, 20]). They reduce to the classical non-crossing partitions of Kreweras 30 for the irreducible reflection groups of type $A_{n}$ (i.e., the symmetric groups) and to Reiner's 32 type $B_{n}$ non-crossing partitions for the irreducible reflections groups of type $B_{n}$. (They differ, however, from the type $D_{n}$ non-crossing partitions of [32.) The subject has been enriched by Armstrong through the introduction of his generalised non-crossing partitions for reflection groups in [1. In the symmetric group case, these reduce to the $m$-divisible non-crossing partitions of Edelman [18, while they produce new combinatorial objects already for the reflection groups of type $B_{n}$. Again, these generalised non-crossing partitions possess numerous beautiful properties and seem to relate to several other objects of combinatorics and algebra, most notably to the generalised cluster complex of Fomin and Reading [19] (cf. [1, 6, 17, 20, 27, 28, 29, 36, 37, 38]).

From a technical point of view, the main subject matter of the present paper is the computation of the number of certain factorisations of the Coxeter element of a reflection group. These decomposition numbers, as we shall call them from now on (see Section 2 for the precise definition), arose in 27, 28, where it was shown that they play a crucial role in the computation of enumerative invariants of (generalised) non-crossing partitions. Moreover, in these two papers the decomposition numbers for the exceptional reflection groups have been computed, and it was pointed out that the decomposition numbers in type $A_{n}$ (i.e., the decomposition numbers for the symmetric groups) had been earlier computed by Goulden and Jackson in 23. Here we explain how the decomposition numbers in type $B_{n}$ can be extracted from results of Bóna, Bousquet, Labelle and Leroux [12] on the enumeration of certain planar maps, and we find formulae for the decomposition numbers in type $D_{n}$, thus completing the project of computing the decomposition numbers for all the irreducible reflection groups.

The main goal of the present paper, however, is to access the enumerative theory of the generalised non-crossing partitions of Armstrong via these decomposition numbers. Indeed, one finds numerous enumerative results on ordinary and generalised non-crossing partitions in the literature (cf. 1, 2, 5, 8, 9, 18, 30, 32, 37): results on the total number of (generalised) non-crossing partitions of a given size, of those with a fixed number of blocks, of those with a given block structure, results on the number of (multi-)chains of a given length in a given poset of (generalised) non-crossing partitions, results on rank-selected chain enumeration (that is, results on the number of chains in which the ranks of the elements of the chains have been fixed), etc. We show that not only can all these results be rederived from our decomposition numbers, we are also able to find several new enumerative results. In this regard, the most general type of result that we find is formulae for the number of (multi-)chains $\pi_{1} \leq \pi_{2} \leq \cdots \leq \pi_{l-1}$ in the poset of non-crossing partitions of type $A_{n}, B_{n}$, respectively $D_{n}$, in which the block structure of $\pi_{1}$ is fixed as well as the ranks of $\pi_{2}, \ldots, \pi_{l-1}$. Even the corresponding result in type $A_{n}$, for the noncrossing partitions of Kreweras, is new. Furthermore, from the result in type $D_{n}$, by a suitable summation, we are able to find a formula for the rank-selected chain enumeration in the poset of generalised non-crossing partitions of type $D_{n}$, thus 
generalising the earlier formula of Athanasiadis and Reiner [5] for the rank-selected chain enumeration of "ordinary" non-crossing partitions of type $D_{n}$. In conjunction with the results from [27, 28, this generalisation in turn allows us to complete a computational case-by-case proof of Armstrong's " $F=M$ Conjecture" [1, Conjecture 5.3.2] predicting a surprising relationship between a certain face count in the generalised cluster complex of Fomin and Reading and the Möbius function in the poset of generalised non-crossing partitions of Armstrong. (A case-free proof had been found earlier by Tzanaki in [38.) Our results also allow us to address another conjecture of Armstrong [1, Conj. 3.5.13] on maximal intervals containing a random multichain in the poset of generalised non-crossing partitions. We show that the conjecture is indeed true for types $A_{n}$ and $B_{n}$, but that it fails for type $D_{n}$ (and we suspect that it will also fail for most of the exceptional types).

We remark that a totally different approach to the enumerative theory of (generalised) non-crossing partitions is proposed in 29. This approach is, however, completely combinatorial and avoids, in particular, reflection groups. It is, therefore, not capable of computing our decomposition numbers or anything else which is intrinsic to the combinatorics of reflection groups. A similar remark applies to [31, Theorem 4.1], where a remarkable uniform recurrence is found for rank-selected chain enumeration in the generalised non-crossing partitions of any type. It could be used, for example, for verifying our result in Corollary 19 on the rank-selected chain enumeration in the generalised non-crossing partitions of type $D_{n}$, but it is not capable of computing our decomposition numbers or of verifying results with restrictions on block structure.

Our paper is organised as follows. In the next section we define the decomposition numbers for finite reflection groups from 27, 28, the central objects in our paper, together with a combinatorial variant, which depends on combinatorial realisations of non-crossing partitions, which we also explain in the same section. This is followed by an intermediate section in which we collect together some auxiliary results that will be needed later. In Section 4, we recall Goulden and Jackson's formula 23 for the full rank decomposition numbers of type $A_{n}$, together with the formula from [28, Theorem 10] that it implies for the decomposition numbers of type $A_{n}$ of arbitrary rank. The purpose of Section 5 is to explain how formulae for the decomposition numbers of type $B_{n}$ can be extracted from results of Bóna, Bousquet, Labelle and Leroux in [12. The type $D_{n}$ decomposition numbers are computed in Section [ The approach that we follow is, essentially, the approach of Goulden and Jackson in 23: we translate the counting problem into the problem of enumerating certain maps. This problem is then solved by a combinatorial decomposition of these maps, translating the decomposition into a system of equations for corresponding generating functions, and finally solving this system with the help of the multidimensional Lagrange inversion formula of Good. Sections 711 form the "applications" part of the paper. In the preparatory section, Section 7 we recall the definition of the generalised non-crossing partitions of Armstrong and explain the combinatorial realisations of the generalised non-crossing partitions for the types $A_{n}, B_{n}$, and $D_{n}$ from [1] and 29. The bulk of the applications is contained in Section 8, where we present three theorems, Theorems [11, 13, and 15, on the number of factorisations of a Coxeter element of type $A_{n}, B_{n}$, respectively $D_{n}$, with less stringent restrictions on the factors than for the decomposition numbers. These theorems result from our formulae for the (combinatorial) decomposition 
numbers upon appropriate summations. Subsequently, it is shown that the corresponding formulae imply all known enumeration results on non-crossing partitions and generalised non-crossing partitions, plus several new ones; see Corollaries 12 , 14, 16, 19 and the accompanying remarks. Section 9 presents the announced computational proof of the $F=M$ (ex-)Conjecture for type $D_{n}$, based on our formula in Corollary 19 for the rank-selected chain enumeration in the poset of generalised non-crossing partitions of type $D_{n}$, while Section 10 addresses Conjecture 3.5.13 from [1, showing that it does not hold in general since it fails in type $D_{n}$. In the final section, Section 11 we point out that the decomposition numbers do not only allow one to derive enumerative results for the generalised non-crossing partitions of the classical types, they also provide all the means for doing this for the exceptional types. For the convenience of the reader, we list the values of the decomposition numbers for the exceptional types that have been computed in [27, 28] in an appendix.

In concluding the Introduction, we want to attract the reader's attention to the fact that many of the formulae presented here are very combinatorial in nature (see Sections 4 5 8). This raises the natural question as to whether it is possible to find combinatorial proofs for them. Indeed, a combinatorial (and, in fact, almost bijective) proof of the formula of Goulden and Jackson, presented here in Theorem 5 , has been given by Bousquet, Chauve and Schaeffer in 13. Moreover, most of the proofs for the known enumeration results on (generalised) non-crossing partitions presented in 1, 2, 15, 18, 32, are combinatorial. On the other hand, to our knowledge so far no one has given a combinatorial proof for Theorem 7 , the formula for the decomposition numbers of type $B_{n}$, essentially due to Bóna, Bousquet, Labelle and Leroux [12], although we believe that this should be possible by modifying the ideas from [13. There are also other formulae in our paper (see e.g. Corollaries 12 and 14, and Eqs. (6.1) and (8.33) which seem amenable to combinatorial proofs. However, to find combinatorial proofs for our type $D_{n}$ results (cf. in particular Theorem 9(ii) and Corollaries 16 [19) seems rather hopeless to us.

\section{DeCOMPosition numbers For Finite CoXeter Groups}

In this section, we introduce the decomposition numbers from 27, 28, which are (Coxeter) group-theoretical in nature, plus combinatorial variants for Coxeter groups of types $B_{n}$ and $D_{n}$, which will be important in combinatorial applications. These variants depend on the combinatorial realisation of these Coxeter groups, which we also explain here.

Let $\Phi$ be a finite root system of rank $n$. (We refer the reader to 24 for all terminology on root systems.) For an element $\alpha \in \Phi$, let $t_{\alpha}$ denote the corresponding reflection in the central hyperplane perpendicular to $\alpha$. Let $W=W(\Phi)$ be the group generated by these reflections. As is well known (cf. e.g. [24, Sec. 6.4]), any such reflection group is at the same time a finite Coxeter group, and all finite Coxeter groups can be realised in this way. By definition, any element $w$ of $W$ can be represented as a product $w=t_{1} t_{2} \cdots t_{\ell}$, where the $t_{i}$ 's are reflections. We call the minimal number of reflections which is needed for such a product representation the absolute length of $w$, and we denote it by $\ell_{T}(w)$. We then define the absolute order on $W$, denoted by $\leq_{T}$, via

$$
u \leq_{T} w \text { if and only if } \ell_{T}(w)=\ell_{T}(u)+\ell_{T}\left(u^{-1} w\right) .
$$


As is well known and easy to see, this is equivalent to the statement that every shortest representation of $u$ by reflections occurs as an initial segment in some shortest product representation of $w$ by reflections.

Now, for a finite root system $\Phi$ of rank $n$, types $T_{1}, T_{2}, \ldots, T_{d}$ (in the sense of the classification of finite Coxeter groups), and a Coxeter element c, the decomposition number $N_{\Phi}\left(T_{1}, T_{2}, \ldots, T_{d}\right)$ is defined as the number of "minimal" products $c_{1} c_{2} \cdots c_{d}$ less than or equal to $c$ in absolute order, "minimal" meaning that $\ell_{T}\left(c_{1}\right)+\ell_{T}\left(c_{2}\right)+\cdots+\ell_{T}\left(c_{d}\right)=\ell_{T}\left(c_{1} c_{2} \cdots c_{d}\right)$, such that, for $i=1,2, \ldots, d$, the type of $c_{i}$ as a parabolic Coxeter element is $T_{i}$. (Here, the term "parabolic Coxeter element" means a Coxeter element in some parabolic subgroup. The reader should recall that it follows from [8. Lemma 1.4.3] that any element $c_{i}$ is indeed a Coxeter element in a parabolic subgroup of $W=W(\Phi)$. By definition, the type of $c_{i}$ is the type of this parabolic subgroup. The reader should also note that, because of the rewriting

$$
c_{1} c_{2} \cdots c_{d}=c_{i}\left(c_{i}^{-1} c_{1} c_{i}\right)\left(c_{i}^{-1} c_{2} c_{i}\right) \cdots\left(c_{i}^{-1} c_{i-1} c_{i}\right) c_{i+1} \cdots c_{d},
$$

any $c_{i}$ in such a minimal product $c_{1} c_{2} \cdots c_{d} \leq_{T} c$ is itself $\leq_{T} c$.) It is easy to see that the decomposition numbers are independent of the choice of the Coxeter element $c$. (This follows from the well-known fact that any two Coxeter elements are conjugate to each other; cf. [24, Sec. 3.16].)

The decomposition numbers satisfy several linear relations between themselves. First of all, the number $N_{\Phi}\left(T_{1}, T_{2}, \ldots, T_{d}\right)$ is independent of the order of the types $T_{1}, T_{2}, \ldots, T_{d}$; that is, we have

$$
N_{\Phi}\left(T_{\sigma(1)}, T_{\sigma(2)}, \ldots, T_{\sigma(d)}\right)=N_{\Phi}\left(T_{1}, T_{2}, \ldots, T_{d}\right)
$$

for every permutation $\sigma$ of $\{1,2, \ldots, d\}$. This is, in fact, a consequence of the rewriting (2.1). Furthermore, by the definition of these numbers, those of "lower rank" can be computed from those of "full rank." To be precise, we have

$$
N_{\Phi}\left(T_{1}, T_{2}, \ldots, T_{d}\right)=\sum_{T} N_{\Phi}\left(T_{1}, T_{2}, \ldots, T_{d}, T\right),
$$

where the sum is taken over all types $T$ of $\operatorname{rank} n-\operatorname{rk} T_{1}-\operatorname{rk} T_{2}-\cdots-\operatorname{rk} T_{d}$ (with $\operatorname{rk} T$ denoting the rank of the root system $\Psi$ of type $T$, and $n$ still denoting the rank of the fixed root system $\Phi$; for later use we record that

$$
\ell_{T}\left(w_{0}\right)=\operatorname{rk} T_{0}
$$

for any parabolic Coxeter element $w_{0}$ of type $T_{0}$ ).

The decomposition numbers for the exceptional types have been computed in 27, 28. For the benefit of the reader, we reproduce these numbers in the appendix. The decomposition numbers for type $A_{n}$ are given in Section 4 , the ones for type $B_{n}$ are computed in Section [5, while the ones for type $D_{n}$ are computed in Section 6 .

Next we introduce variants of the above decomposition numbers for the types $B_{n}$ and $D_{n}$, which depend on the combinatorial realisation of the Coxeter groups of these types.

As is well-known, the reflection group $W\left(A_{n}\right)$ can be realised as the symmetric group $S_{n+1}$ on $\{1,2, \ldots, n+1\}$. The reflection groups $W\left(B_{n}\right)$ and $W\left(D_{n}\right)$, on the other hand, can be realised as subgroups of the symmetric group on $2 n$ elements. (See e.g. [11, Sections 8.1 and 8.2].) Namely, the reflection group $W\left(B_{n}\right)$ can be 
realised as the subgroup of the group of all permutations $\pi$ of

$$
\{1,2, \ldots, n, \overline{1}, \overline{2}, \ldots, \bar{n}\}
$$

satisfying the property

$$
\pi(\bar{i})=\overline{\pi(i)} .
$$

(Here, and in what follows, $\overline{\bar{i}}$ is identified with $i$ for all $i$.) In this realisation, there is an analogue of the disjoint cycle decomposition of permutations. Namely, every $\pi \in W\left(B_{n}\right)$ can be decomposed as

$$
\pi=\kappa_{1} \kappa_{2} \cdots \kappa_{s}
$$

where, for $i=1,2, \ldots, s, \kappa_{i}$ is of one of two possible types of "cycles": a type $A$ cycle, by which we mean a permutation of the form

$$
\left(\left(a_{1}, a_{2}, \ldots, a_{k}\right)\right):=\left(a_{1}, a_{2}, \ldots, a_{k}\right)\left(\overline{a_{1}}, \overline{a_{2}}, \ldots, \overline{a_{k}}\right),
$$

or a type $B$ cycle, by which we mean a permutation of the form

$$
\left[a_{1}, a_{2}, \ldots, a_{k}\right]:=\left(a_{1}, a_{2}, \ldots, a_{k}, \overline{a_{1}}, \overline{a_{2}}, \ldots, \overline{a_{k}}\right),
$$

$a_{1}, a_{2}, \ldots, a_{k} \in\{1,2, \ldots, n, \overline{1}, \overline{2}, \ldots, \bar{n}\}$. (Here we adopt notation from 15.) In both cases, we call $k$ the length of the "cycle." The decomposition (2.6) is unique up to a reordering of the $\kappa_{i}$ 's.

We call a type $A$ cycle of length $k$ of combinatorial type $A_{k-1}$, while we call a type $B$ cycle of length $k$ of combinatorial type $B_{k}, k=1,2, \ldots$ The reader should observe that, when regarded as a parabolic Coxeter element, for $k \geq 2$ a type $A$ cycle of length $k$ has type $A_{k-1}$, while a type $B$ cycle of length $k$ has type $B_{k}$. However, a type $B$ cycle of length 1 , that is, a permutation of the form $(i, \bar{i})$, has type $A_{1}$ when regarded as a parabolic Coxeter element, while we say that it has combinatorial type $B_{1}$. (The reader should recall that, in the classification of finite Coxeter groups, the type $B_{1}$ does not occur, respectively, that sometimes $B_{1}$ is identified with $A_{1}$. Here, when we speak of "combinatorial type," we $d o$ distinguish between $A_{1}$ and $B_{1}$. For example, the "cycles" $((1,2))=(1,2)(\overline{1}, \overline{2})$ or $((\overline{1}, 2))=(\overline{1}, 2)(1, \overline{2})$ have combinatorial type $A_{1}$, whereas the cycles $[1]=(1, \overline{1})$ or $[2]=(2, \overline{2})$ have combinatorial type $B_{1}$.)

As a Coxeter element for $W\left(B_{n}\right)$, we choose

$$
c=(1,2, \ldots, n, \overline{1}, \overline{2}, \ldots, \bar{n})=[1,2, \ldots, n] .
$$

Now, given combinatorial types $T_{1}, T_{2}, \ldots, T_{d}$, each of which is a product of $A_{k}$ 's and $B_{k}$ 's, $k=1,2, \ldots$, the combinatorial decomposition number $N_{B_{n}}^{\text {comb }}\left(T_{1}, T_{2}, \ldots, T_{d}\right)$ is defined as the number of minimal products $c_{1} c_{2} \cdots c_{d}$ less than or equal to $c$ in absolute order, where "minimal" has the same meaning as above, such that for $i=1,2, \ldots, d$ the combinatorial type of $c_{i}$ is $T_{i}$. Because of (2.1), the combinatorial decomposition numbers $N_{B_{n}}^{\text {comb }}\left(T_{1}, T_{2}, \ldots, T_{d}\right)$ also satisfy (2.2) and (2.3).

The reflection group $W\left(D_{n}\right)$ can be realised as the subgroup of the group of all permutations $\pi$ of $\{1,2, \ldots, n, \overline{1}, \overline{2}, \ldots, \bar{n}\}$ satisfying (2.5) and the property that an even number of elements from $\{1,2, \ldots, n\}$ is mapped to an element of negative sign. (Here, the elements $1,2, \ldots, n$ are considered to have sign + , while the elements $\overline{1}, \overline{2}, \ldots, \bar{n}$ are considered to have sign -.) Since $W\left(D_{n}\right)$ is a subgroup of $W\left(B_{n}\right)$, and since the above realisation of $W\left(D_{n}\right)$ is contained as a subset in the realisation of $W\left(B_{n}\right)$ that we just described, any $\pi \in W\left(D_{n}\right)$ can be decomposed as in (2.6), where, for $i=1,2, \ldots, d, \kappa_{i}$ is either a type $A$ or a type $B$ cycle. Requiring that 
$\pi$ is in the subgroup $W\left(D_{n}\right)$ of $W\left(B_{n}\right)$ is equivalent to requiring that there is an even number of type $B$ cycles in the decomposition (2.6). Again, the decomposition (2.6) for $\pi \in W\left(D_{n}\right)$ is unique up to a reordering of the $\kappa_{i}$ 's.

As a Coxeter element, we choose

$$
c=(1,2, \ldots, n-1, \overline{1}, \overline{2}, \ldots, \overline{n-1})(n, \bar{n})=[1,2, \ldots, n-1][n] .
$$

We shall be entirely concerned with elements $\pi$ of $W\left(D_{n}\right)$ which are less than or equal to $c$. It is not difficult to see (and it is shown in [5, Sec. 3]) that the unique factorisation of any such element $\pi$ has either 0 or 2 type $B$ cycles, and in the latter case one of the type $B$ cycles is $[n]=(n, \bar{n})$. In this latter case, in abuse of terminology, we call the product of these two type $B$ cycles, $\left[a_{1}, a_{2}, \ldots, a_{k-1}\right][n]$ say, a "cycle" of combinatorial type $D_{k}$. More generally, we shall say for any product of two disjoint type $B$ cycles of the form

$$
\left[a_{1}, a_{2}, \ldots, a_{k-1}\right]\left[a_{k}\right]
$$

that it is a "cycle" of combinatorial type $D_{k}$. The reader should observe that, when regarded as a parabolic Coxeter element, for $k \geq 4$ an element of the form (2.9) has type $D_{k}$. However, if $k=3$, it has type $A_{3}$ when regarded as a parabolic Coxeter element, while we say that it has combinatorial type $D_{3}$, and, if $k=2$, it has type $A_{1}^{2}$ when regarded as a parabolic Coxeter element, while we say that it has combinatorial type $D_{2}$. (The reader should recall that, in the classification of finite Coxeter groups, the types $D_{3}$ and $D_{2}$ do not occur, respectively, that sometimes $D_{3}$ is identified with $A_{3}, D_{2}$ being identified with $A_{1}^{2}$. Here, when we speak of "combinatorial type," we do distinguish between $D_{3}$ and $A_{3}$, and between $D_{2}$ and $A_{1}^{2}$.)

Now, given combinatorial types $T_{1}, T_{2}, \ldots, T_{d}$, each of which is a product of $A_{k}$ 's and $D_{k}$ 's, $k=1,2, \ldots$, the combinatorial decomposition number $N_{D_{n}}^{\text {comb }}\left(T_{1}, T_{2}, \ldots\right.$, $\left.T_{d}\right)$ is defined as the number of minimal products $c_{1} c_{2} \cdots c_{d}$ less than or equal to $c$ in absolute order, where "minimal" has the same meaning as above, such that for $i=1,2, \ldots, d$ the combinatorial type of $c_{i}$ is $T_{i}$. Because of (2.1), the combinatorial decomposition numbers $N_{D_{n}}^{\text {comb }}\left(T_{1}, T_{2}, \ldots, T_{d}\right)$ also satisfy (2.2) and (2.3).

\section{Auxiliary Results}

In our computations in the proof of Theorem 9, leading to the determination of the decomposition numbers of type $D_{n}$, we need to apply the Lagrange-Good inversion formula 22. (see also [26, Sec. 5] and the references cited therein). We recall it here for the convenience of the reader. In doing so, we use standard multi-index notation. Namely, given a positive integer $d$, and vectors $\mathbf{z}=\left(z_{1}, z_{2}, \ldots, z_{d}\right)$ and $\mathbf{n}=\left(n_{1}, n_{2}, \ldots, n_{d}\right)$, we write $\mathbf{z}^{\mathbf{n}}$ for $z_{1}^{n_{1}} z_{2}^{n_{2}} \cdots z_{d}^{n_{d}}$. Furthermore, in abuse of notation, given a formal power series $f$ in $d$ variables, $f(\mathbf{z})$ stands for $f\left(z_{1}, z_{2}, \ldots, z_{d}\right)$. Moreover, given $d$ formal power series $f_{1}, f_{2}, \ldots, f_{d}$ in $d$ variables, $\mathbf{f}^{\mathbf{n}}(\mathbf{z})$ is short for

$$
f_{1}^{n_{1}}\left(z_{1}, z_{2}, \ldots, z_{d}\right) f_{2}^{n_{2}}\left(z_{1}, z_{2}, \ldots, z_{d}\right) \cdots f_{d}^{n_{d}}\left(z_{1}, z_{2}, \ldots, z_{d}\right) .
$$

Finally, if $\mathbf{m}=\left(m_{1}, m_{2}, \ldots, m_{d}\right)$ is another vector, then $\mathbf{m}+\mathbf{n}$ is short for $\left(m_{1}+\right.$ $\left.n_{1}, m_{2}+n_{2}, \ldots, m_{d}+n_{d}\right)$. Notation such as $\mathbf{m}-\mathbf{n}$ has to be interpreted in a similar way.

Theorem 1 (Lagrange-Good inversion). Let $d$ be a positive integer, and let $f_{1}(\mathbf{z})$, $f_{2}(\mathbf{z}), \ldots, f_{d}(\mathbf{z})$ be a formal power series in $\mathbf{z}=\left(z_{1}, z_{2}, \ldots, z_{d}\right)$ with the property that, for all $i, f_{i}(\mathbf{z})$ is of the form $z_{i} / \varphi_{i}(\mathbf{z})$ for some formal power series $\varphi_{i}(\mathbf{z})$ 
with $\varphi_{i}(0,0, \ldots, 0) \neq 0$. Then, if we expand a formal power series $g(\mathbf{z})$ in terms of powers of the $f_{i}(\mathbf{z})$,

$$
g(\mathbf{z})=\sum_{\mathbf{n}} \gamma_{\mathbf{n}} \mathbf{f}^{\mathbf{n}}(\mathbf{z})
$$

the coefficients $\gamma_{\mathbf{n}}$ are given by

$$
\gamma_{\mathbf{n}}=\left\langle\mathbf{z}^{-e}\right\rangle g(\mathbf{z}) \mathbf{f}^{-\mathbf{n}-e}(\mathbf{z}) \operatorname{det}_{1 \leq i, j \leq d}\left(\frac{\partial f_{i}}{\partial z_{j}}(\mathbf{z})\right),
$$

where $\mathbf{e}=(1,1, \ldots, 1)$, where the sum in (3.1) runs over all $d$-tuples $\mathbf{n}$ of nonnegative integers, and where $\left\langle\mathbf{z}^{\mathbf{m}}\right\rangle h(\mathbf{z})$ denotes the coefficient of $\mathbf{z}^{\mathbf{m}}$ in the formal Laurent series $h(\mathbf{z})$.

Next, we prove a determinant lemma and a corollary, both of which will also be used in the proof of Theorem 9 ,

Lemma 2. Let $d$ be a positive integer, and let $X_{1}, X_{2}, \ldots, X_{d}, Y_{2}, Y_{3}, \ldots, Y_{d}$ be indeterminates. Then

$$
\operatorname{det}_{1 \leq i, j \leq d}\left(\left\{\begin{array}{cc}
1-\chi(1 \neq j) \frac{Y_{j}}{X_{1}}, & i=1 \\
1-\chi(i \neq j) \frac{Y_{i}}{X_{i}}, & i \geq 2
\end{array}\right\}\right)=\frac{\left(\sum_{i=1}^{d} X_{i}-\sum_{i=2}^{d} Y_{i}\right) Y_{2} Y_{3} \cdots Y_{d}}{X_{1} X_{2} \cdots X_{d}},
$$

where $\chi(\mathcal{S})=1$ if $\mathcal{S}$ is true and $\chi(\mathcal{S})=0$ otherwise.

Proof. By using multilinearity in the rows, we rewrite the determinant on the lefthand side of (3.2) as

$$
\frac{1}{X_{1} X_{2} \cdots X_{d}} \operatorname{det}_{1 \leq i, j \leq d}\left(\left\{\begin{array}{cc}
X_{1}-\chi(1 \neq j) Y_{j}, & i=1 \\
X_{i}-\chi(i \neq j) Y_{i}, & i \geq 2
\end{array}\right\}\right) .
$$

Next, we subtract the first column from all other columns. As a result, we obtain the determinant

$$
\frac{1}{X_{1} X_{2} \cdots X_{d}} \operatorname{det}_{1 \leq i, j \leq d}\left(\left\{\begin{array}{cc}
X_{1}, & i=j=1 \\
-Y_{j}, & i=1 \text { and } j \geq 2 \\
X_{i}-Y_{i}, & i \geq 2 \text { and } j=1 \\
\chi(i=j) Y_{i}, & i, j \geq 2
\end{array}\right\}\right) .
$$

Now we add rows $2,3, \ldots, d$ to the first row. After that, our determinant becomes lower triangular, with the entry in the first row and column equal to $\sum_{i=1}^{d} X_{i}-$ $\sum_{i=2}^{d} Y_{i}$ and with the diagonal entry in row $i, i \geq 2$, equal to $Y_{i}$. Hence, we obtain the claimed result.

Corollary 3. Let $d$ and $r$ be positive integers, $1 \leq r \leq d$, and let $X_{1}, X_{2}, \ldots, X_{d}$, $Y$ and $Z$ be indeterminates. Then, with notation as in Lemma 2 ,

$$
\begin{array}{r}
\underset{1 \leq i, j \leq d}{\operatorname{det}}\left(\left\{\begin{array}{ll}
1-\chi(r \neq j) \frac{Z}{X_{r}}, & i=r \\
1-\chi(i \neq j) \frac{Y}{X_{i}}, & i \neq r
\end{array}\right\}\right) \\
=\frac{Y^{d-2}\left(Z \sum_{i=1}^{d} X_{i}+(Y-Z) X_{r}-(d-1) Y Z\right)}{X_{1} X_{2} \cdots X_{d}} .
\end{array}
$$


Proof. We write the diagonal entry in the $r$-th row of the determinant in (3.3) as

$$
1=\frac{X_{r}+Y-Z}{X_{r}}-\frac{Y-Z}{X_{r}}
$$

and then use linearity of the determinant in the $r$-th row to decompose the determinant as

$$
\frac{X_{r}+Y-Z}{X_{r}} D_{1}-\frac{Y-Z}{X_{r}} D_{2},
$$

where $D_{1}$ is the determinant in (3.2) with $X_{r}$ replaced by $X_{r}+Y-Z$, and with $Y_{i}=Y$ for all $i$, and where $D_{2}$ is the determinant in (3.2) with $d$ replaced by $d-1$, with $Y_{i}=Y$ for all $i$, and with $X_{i}$ replaced by $X_{i-1}$ for $i=r+1, r+2, \ldots, d$. Hence, using Lemma 2 we deduce that the determinant in (3.3) is equal to

$$
\frac{Y^{d-1}\left(\sum_{i=1}^{d} X_{i}+Y-Z-(d-1) Y\right)}{X_{1} X_{2} \cdots X_{d}}-\frac{(Y-Z) Y^{d-2}\left(\sum_{i=1}^{d} X_{i}-X_{r}-(d-2) Y\right)}{X_{1} X_{2} \cdots X_{d}} .
$$

Little simplification then leads to (3.3).

We end this section with a summation lemma, which we shall need in Sections 5 and 6 in order to compute the $B_{n}$, respectively $D_{n}$, decomposition numbers of arbitrary rank from those of full rank. We shall also use it in Section 8 to derive enumerative results for (generalised) non-crossing partitions from our formulae for the decomposition numbers.

Lemma 4. Let $M$ and $r$ be non-negative integers. Then

$$
\sum_{m_{1}+2 m_{2}+\cdots+r m_{r}=r}\left(\begin{array}{c}
M \\
m_{1}, m_{2}, \ldots, m_{r}
\end{array}\right)=\left(\begin{array}{c}
M+r-1 \\
r
\end{array}\right),
$$

where the multinomial coefficient is defined by

$$
\left(\begin{array}{c}
M \\
m_{1}, m_{2}, \ldots, m_{r}
\end{array}\right)=\frac{M !}{m_{1} ! m_{2} ! \cdots m_{r} !\left(M-m_{1}-m_{2}-\cdots-m_{r}\right) !} .
$$

Proof. The identity results directly by comparing coefficients of $z^{r}$ on both sides of the identity

$$
\left(1+z+z^{2}+z^{3}+\cdots\right)^{M}=(1-z)^{-M} .
$$

\section{Decomposition numbers for type $A$}

As was pointed out in [28, Sec. 10], the decomposition numbers for type $A_{n}$ have already been computed by Goulden and Jackson in [23, Theorem 3.2], albeit using a somewhat different language. (The condition on the sum $l\left(\alpha_{1}\right)+l\left(\alpha_{2}\right)+\cdots+l\left(\alpha_{m}\right)$ is misstated throughout the latter paper. It should be replaced by $l\left(\alpha_{1}\right)+l\left(\alpha_{2}\right)+$ $\cdots+l\left(\alpha_{m}\right)=(m-1) n+1$.) In our terminology, their result reads as follows.

Theorem 5. Let $T_{1}, T_{2}, \ldots, T_{d}$ be types with $\operatorname{rk} T_{1}+\operatorname{rk} T_{2}+\cdots+\operatorname{rk} T_{d}=n$, where

$$
T_{i}=A_{1}^{m_{1}^{(i)}} * A_{2}^{m_{2}^{(i)}} * \cdots * A_{n}^{m_{n}^{(i)}}, \quad i=1,2, \ldots, d .
$$

Then

$$
N_{A_{n}}\left(T_{1}, T_{2}, \ldots, T_{d}\right)=(n+1)^{d-1} \prod_{i=1}^{d} \frac{1}{n-\operatorname{rk} T_{i}+1}\left(\begin{array}{c}
n-\operatorname{rk} T_{i}+1 \\
m_{1}^{(i)}, m_{2}^{(i)}, \ldots, m_{n}^{(i)}
\end{array}\right),
$$

where the multinomial coefficient is defined as in Lemma 4 . 
Here we have used Stembridge's [35] notation for the decomposition of types into a product of irreducibles; for example, the equation $T=A_{2}^{3} * A_{5}$ means that the root system of type $T$ decomposes into the orthogonal product of 3 copies of root systems of type $A_{2}$ and one copy of the root system of type $A_{5}$.

It was shown in [28, Theorem 10] that, upon applying the summation formula in Lemma 4 to the result in Theorem 5 in a suitable manner, one obtains a compact formula for all type $A_{n}$ decomposition numbers.

Theorem 6. Let the types $T_{1}, T_{2}, \ldots, T_{d}$ be given, where

$$
T_{i}=A_{1}^{m_{1}^{(i)}} * A_{2}^{m_{2}^{(i)}} * \cdots * A_{n}^{m_{n}^{(i)}}, \quad i=1,2, \ldots, d .
$$

Then

$$
\begin{aligned}
N_{A_{n}}\left(T_{1}, T_{2}, \ldots, T_{d}\right)=(n+1)^{d-1} & \left(\begin{array}{c}
n+1 \\
\operatorname{rk} T_{1}+\operatorname{rk} T_{2}+\cdots+\operatorname{rk} T_{d}+1
\end{array}\right) \\
& \times \prod_{i=1}^{d} \frac{1}{n-\operatorname{rk} T_{i}+1}\left(\begin{array}{c}
n-\operatorname{rk} T_{i}+1 \\
m_{1}^{(i)}, m_{2}^{(i)}, \ldots, m_{n}^{(i)}
\end{array}\right),
\end{aligned}
$$

where the multinomial coefficient is defined as in Lemma 4. All other decomposition numbers $N_{A_{n}}\left(T_{1}, T_{2}, \ldots, T_{d}\right)$ are zero.

\section{Decomposition numbers For type $B$}

In this section we compute the decomposition numbers in type $B_{n}$. We show that one can extract the corresponding formulae from results of Bóna, Bousquet, Labelle and Leroux 12 on the enumeration of certain planar maps, which they call $m$-ary cacti. While reading the statement of the theorem, the reader should recall from Section 2 the distinction between group-theoretic and combinatorial decomposition numbers.

Theorem 7. (i) If $T_{1}, T_{2}, \ldots, T_{d}$ are types with $\operatorname{rk} T_{1}+\operatorname{rk} T_{2}+\cdots+\operatorname{rk} T_{d}=n$, where

$$
T_{i}=A_{1}^{m_{1}^{(i)}} * A_{2}^{m_{2}^{(i)}} * \cdots * A_{n}^{m_{n}^{(i)}}, \quad i=1,2, \ldots, j-1, j+1, \ldots, d,
$$

and

$$
T_{j}=B_{\alpha} * A_{1}^{m_{1}^{(j)}} * A_{2}^{m_{2}^{(j)}} * \cdots * A_{n}^{m_{n}^{(j)}},
$$

for some $\alpha \geq 1$, then

$$
\begin{aligned}
N_{B_{n}}^{c o m b}\left(T_{1}, T_{2}, \ldots, T_{d}\right)= & n^{d-1}\left(\begin{array}{c}
n-\operatorname{rk} T_{j} \\
m_{1}^{(j)}, m_{2}^{(j)}, \ldots, m_{n}^{(j)}
\end{array}\right) \\
& \times \prod_{\substack{i=1 \\
i \neq j}}^{d} \frac{1}{n-\operatorname{rk} T_{i}}\left(\begin{array}{c}
n-\operatorname{rk} T_{i} \\
m_{1}^{(i)}, m_{2}^{(i)}, \ldots, m_{n}^{(i)}
\end{array}\right),
\end{aligned}
$$

where the multinomial coefficient is defined as in Lemma 4. For $\alpha \geq 2$, the number $N_{B_{n}}\left(T_{1}, T_{2}, \ldots, T_{d}\right)$ is given by the same formula.

(ii) If $T_{1}, T_{2}, \ldots, T_{d}$ are types with $\operatorname{rk} T_{1}+\operatorname{rk} T_{2}+\cdots+\operatorname{rk} T_{d}=n$, where

$$
T_{i}=A_{1}^{m_{1}^{(i)}} * A_{2}^{m_{2}^{(i)}} * \cdots * A_{n}^{m_{n}^{(i)}}, \quad i=1,2, \ldots, d
$$


then

$$
\begin{aligned}
N_{B_{n}}\left(T_{1}, T_{2}, \ldots, T_{d}\right)= & n^{d-1}\left(\prod_{i=1}^{d} \frac{1}{n-\operatorname{rk} T_{i}}\left(\begin{array}{c}
n-\operatorname{rk} T_{i} \\
m_{1}^{(i)}, m_{2}^{(i)}, \ldots, m_{n}^{(i)}
\end{array}\right)\right) \\
& \times \sum_{j=1}^{d} \frac{m_{1}^{(j)}\left(n-\operatorname{rk} T_{j}\right)}{m_{0}^{(j)}+1}
\end{aligned}
$$

where $m_{0}^{(j)}=n-\operatorname{rk} T_{j}-\sum_{s=1}^{n} m_{s}^{(j)}$.

(iii) All of the other decomposition numbers $N_{B_{n}}\left(T_{1}, T_{2}, \ldots, T_{d}\right)$ and $N_{B_{n}}^{\text {comb }}\left(T_{1}, T_{2}, \ldots, T_{d}\right)$ with $\operatorname{rk} T_{1}+\operatorname{rk} T_{2}+\cdots+\operatorname{rk} T_{d}=n$ are zero.

Proof. Determining the decomposition numbers

$$
N_{B_{n}}\left(T_{1}, T_{2}, \ldots, T_{d}\right)=N_{B_{n}}\left(T_{d}, \ldots, T_{2}, T_{1}\right)
$$

(recall (2.2) $)$, respectively

$$
N_{B_{n}}^{\text {comb }}\left(T_{1}, T_{2}, \ldots, T_{d}\right)=N_{B_{n}}^{\text {comb }}\left(T_{d}, \ldots, T_{2}, T_{1}\right),
$$

amounts to counting all possible factorisations

$$
[1,2, \ldots, n]=\sigma_{d} \cdots \sigma_{2} \sigma_{1}
$$

where $\sigma_{i}$ has type $T_{i}$ as a parabolic Coxeter element, respectively has a combinatorial type $T_{i}$. The reader should observe that the factorisation (5.3) is minimal in the sense that

$$
n=\ell_{T}([1,2, \ldots, n])=\ell_{T}\left(\sigma_{1}\right)+\ell_{T}\left(\sigma_{2}\right)+\cdots+\ell_{T}\left(\sigma_{d}\right),
$$

since $\ell_{T}\left(\sigma_{i}\right)=\operatorname{rk} T_{i}$, and since, by our assumption, the sum of the ranks of the $T_{i}$ 's equals $n$. A further observation is that, in a factorisation (5.3), there must be at least one factor $\sigma_{i}$ which contains a type $B$ cycle in its (type $B$ ) disjoint cycle decomposition, because the sign of $[1,2, \ldots, n]$ as an element of the group $S_{2 n}$ of all permutations of $\{1,2, \ldots, n, \overline{1}, \overline{2}, \ldots, \bar{n}\}$ is -1 , while the sign of any type $A$ cycle is +1 .

We first prove claim (iii). Let us assume, by contradiction, that there is a minimal decomposition (5.3) in which, altogether, we find at least two type $B$ cycles in the (type $B$ ) disjoint cycle decompositions of the $\sigma_{i}$ 's. In that case, (5.3) has the form

$$
[1,2, \ldots, n]=u_{1} \kappa_{1} u_{2} \kappa_{2} u_{3},
$$

where $\kappa_{1}$ and $\kappa_{2}$ are two type $B$ cycles, and $u_{1}, u_{2}, u_{3}$ are the factors in between. Moreover, the factorisation (5.4) is minimal, meaning that

$$
n=\ell_{T}\left(u_{1}\right)+\ell_{T}\left(\kappa_{1}\right)+\ell_{T}\left(u_{2}\right)+\ell_{T}\left(\kappa_{2}\right)+\ell_{T}\left(u_{3}\right) .
$$

We may rewrite (5.4) as

$$
[1,2, \ldots, n]=\kappa_{1} \kappa_{2}\left(\kappa_{2}^{-1} \kappa_{1}^{-1} u_{1} \kappa_{1} \kappa_{2}\right)\left(\kappa_{2}^{-1} u_{2} \kappa_{2}\right) u_{3},
$$

or, setting $u_{1}^{\prime}=\kappa_{2}^{-1} \kappa_{1}^{-1} u_{1} \kappa_{1} \kappa_{2}$ and $u_{2}^{\prime}=\kappa_{2}^{-1} u_{2} \kappa_{2}$, as

$$
[1,2, \ldots, n]=\kappa_{1} \kappa_{2} u_{1}^{\prime} u_{2}^{\prime} u_{3} .
$$

This factorisation is still minimal since $u_{1}^{\prime}$ is conjugate to $u_{1}$ and $u_{2}^{\prime}$ is conjugate to $u_{2}$. At this point, we observe that $\kappa_{1}$ must be a cycle of the form (2.8) with $a_{1}<a_{2}<\cdots<a_{k}<\overline{a_{1}}<\overline{a_{2}}<\cdots<\overline{a_{k}}$ in the order $1<2<\cdots<n<\overline{1}<$ $\overline{2}<\cdots<\bar{n}$, because otherwise $\kappa_{1} \not_{T}[1,2, \ldots, n]$, which would contradict (5.6). A 


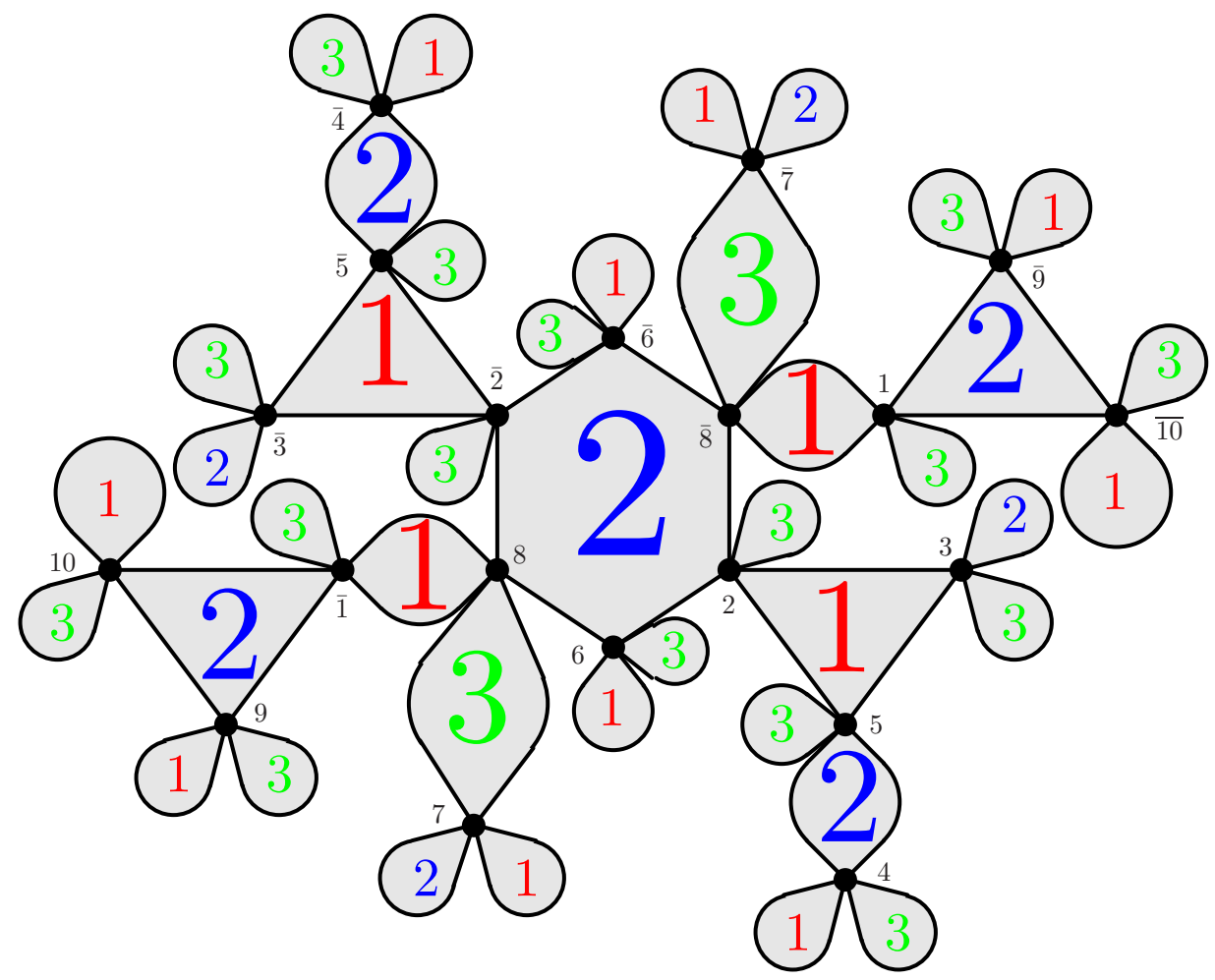

Figure 1. The 3-cactus corresponding to the factorisation (5.7)

similar argument applies to $\kappa_{2}$. Now, if $\kappa_{1}$ and $\kappa_{2}$ are not disjoint, then it is easy to see that $\ell_{T}\left(\kappa_{1} \kappa_{2}\right)<\ell_{T}\left(\kappa_{1}\right)+\ell_{T}\left(\kappa_{2}\right)$; hence

$$
\begin{aligned}
n & =\ell_{T}([1,2, \ldots, n]) \\
& =\ell_{T}\left(\kappa_{1} \kappa_{2} u_{1}^{\prime} u_{2}^{\prime} u_{3}\right) \\
& \leq \ell_{T}\left(\kappa_{1} \kappa_{2}\right)+\ell_{T}\left(u_{1}^{\prime}\right)+\ell_{T}\left(u_{2}^{\prime}\right)+\ell_{T}\left(u_{3}\right) \\
& \leq \ell_{T}\left(\kappa_{1} \kappa_{2}\right)+\ell_{T}\left(u_{1}\right)+\ell_{T}\left(u_{2}\right)+\ell_{T}\left(u_{3}\right) \\
& <\ell_{T}\left(\kappa_{1}\right)+\ell_{T}\left(\kappa_{2}\right)+\ell_{T}\left(u_{1}\right)+\ell_{T}\left(u_{2}\right)+\ell_{T}\left(u_{3}\right),
\end{aligned}
$$

a contradiction to (5.5). If, on the other hand, $\kappa_{1}$ and $\kappa_{2}$ are disjoint, then we can find $i, j \in\{1,2, \ldots, n, \overline{1}, \overline{2}, \ldots, \bar{n}\}$, such that $i<j<\kappa_{1}(i)<\kappa_{2}(j)$ (in the above order of $\{1,2, \ldots, n, \overline{1}, \overline{2}, \ldots, \bar{n}\})$. In other words, if we represent $\kappa_{1}$ and $\kappa_{2}$ in the obvious way in a cyclic diagram (cf. [32, Sec. 2]), then they cross each other. However, in that case we have

$$
\kappa_{1} \kappa_{2} \not \mathbb{Z}_{T}[1,2, \ldots, n],
$$

contradicting the fact that (5.6) is a minimal factorisation. (This is one of the consequences of Biane's group-theoretic characterisation [10, Theorem 1] of noncrossing partitions.)

We now turn to claims (i) and (ii). In what follows, we shall show that the formulae (5.1) and (5.2) follow from results of Bóna, Bousquet, Labelle and Leroux 12 on the enumeration of $m$-ary cacti with a rotational symmetry. In order to 
explain this, we must first define a bijection between minimal factorisations (5.3) and certain planar maps. By a map, we mean a connected graph embedded in the plane such that edges do not intersect except in vertices. The maps which are of relevance here are maps in which faces different from the outer face intersect only in vertices and are coloured with colours from $\{1,2, \ldots, d\}$. Such maps will be referred to as $d$-cacti from now on 1 Examples of 3-cacti can be found in Figures 1 and 2 . In the figures, the faces different from the outer face are the shaded ones. Their colours are indicated by the numbers 1,2 , respectively 3 , placed in the centre of the faces. Figure 1 shows a 3-cactus in which the vertices are labelled, while Figure 2 shows one in which the vertices are not labelled. (The fact that one of the vertices in Figure 2 is marked by a bold dot should be ignored for the moment.)

In what follows, we need the concept of the rotator around a vertex $v$ in a $d$-cactus, which, by definition, is the cyclic list of colours of faces encountered in a clockwise journey around $v$. If, while travelling around $v$, we encounter the colours $b_{1}, b_{2}, \ldots, b_{k}$, in this order, then we will write $\left(b_{1}, b_{2}, \ldots, b_{k}\right)^{\mathrm{O}}$ for the rotator, meaning that $\left(b_{1}, b_{2}, \ldots, b_{k}\right)^{\mathrm{O}}=\left(b_{2}, \ldots, b_{k}, b_{1}\right)^{\mathrm{O}}$, etc. For example, the rotator of all the vertices in the map in Figure 1 is $(1,2,3)^{\mathrm{O}}$.

We illustrate the bijection between minimal factorisations (5.3) and $d$-cacti with an example. Take $n=10$ and $d=3$, and consider the factorisation

$$
[1,2, \ldots, 10]=\sigma_{3} \sigma_{2} \sigma_{1}
$$

where $\sigma_{3}=((7,8)), \sigma_{2}=[2,6,8]((1, \overline{9}, \overline{10}))((4,5))$, and $\sigma_{1}=((1, \overline{8}))((2,3,5))$. For each cycle $\left(a_{1}, a_{2}, \ldots, a_{k}\right)$ (sic!) of $\sigma_{i}$, we create a $k$-gon coloured $i$, and label its vertices $a_{1}, a_{2}, \ldots, a_{k}$ in clockwise order. (The warning "sic!" is there to avoid misunderstandings: for each type $A$ "cycle" $\left(\left(b_{1}, b_{2}, \ldots, b_{k}\right)\right)$ we create two $k$-gons, the vertices of one being labelled $b_{1}, b_{2}, \ldots, b_{k}$, and the vertices of the other being labelled $\overline{b_{1}}, \overline{b_{2}}, \ldots, \overline{b_{k}}$, while for each type $B$ "cycle" $\left[b_{1}, b_{2}, \ldots, b_{k}\right]$ we create one $2 k$ gon with vertices labelled $b_{1}, b_{2}, \ldots, b_{k}, \overline{b_{1}}, \overline{b_{2}}, \ldots, \overline{b_{k}}$.) We glue these polygons into a $d$-cactus, the faces of which are these polygons plus the outer face, by identifying equally labelled vertices such that the rotator of each vertex is $(1,2, \ldots, d)$. Figure 1 shows the outcome of this procedure for the factorisation (5.7).

The fact that the result of the procedure can be realised as a $d$-cactus follows from Euler's formula. Namely, the number of faces corresponding to the polygons is $1+2 \sum_{i=1}^{d} \sum_{k=0}^{n} m_{k}^{(i)}$ (the 1 coming from the polygon corresponding to the type $B$ cycle), the number of edges is $2 \alpha+2 \sum_{i=1}^{d} \sum_{k=0}^{n} m_{k}^{(i)}(k+1)$, and the number of vertices is $2 n$. Hence, if we include the outer face, the number of vertices minus

1 We warn the reader that our terminology deviates from the one in 12 23. We follow loosely the conventions in 25]. To be precise, our $d$-cacti in which the rotator around every vertex is $(1,2, \ldots, d)^{\mathrm{O}}$ are dual to the coloured $d$-cacti in [23], respectively $d$-ary cacti in [12], in the following sense: one is obtained from the other by "interchanging" the roles of vertices and faces; that is, given a $d$-cactus in our sense, one obtains a $d$-cactus in the sense of Goulden and Jackson by shrinking faces to vertices and blowing up vertices of degree $\delta$ to faces with $\delta$ vertices, keeping the incidence relations between faces and vertices. Another minor difference is that colours are arranged in counter-clockwise order in [12, 23, while we arrange colours in clockwise order. 




FIGURE 2. A rotation-symmetric 3-cactus with a marked vertex

the number of edges plus the number of faces is

$$
\begin{aligned}
2 n-2 \alpha-2 \sum_{i=1}^{d} \sum_{k=0}^{n} m_{k}^{(i)}(k+1)+ & 2 \sum_{i=1}^{d} \sum_{k=0}^{n} m_{k}^{(i)}+2 \\
& =2 n+2-2 \alpha-2 \sum_{i=1}^{d} \sum_{k=0}^{n} k \cdot m_{k}^{(i)} \\
& =2 n+2-2 \operatorname{rk} T_{1}-2 \operatorname{rk} T_{2}-\cdots-2 \operatorname{rk} T_{d} \\
& =2,
\end{aligned}
$$

according to our assumption concerning the sum of the ranks of the types $T_{i}$.

We may further simplify this geometric representation of a minimal factorisation (5.3) by deleting all vertex labels and marking the vertex which had label 1 . If this simplification is applied to the 3-cactus in Figure 1, we obtain the 3-cactus in Figure 2. Indeed, the knowledge of which vertex carries label 1 allows us to reconstruct all other vertex labels as follows: starting from the vertex labelled 1 , we travel clockwise along the boundary of the face coloured 1 until we reach the next vertex (that is, we traverse only a single edge); from there, we travel clockwise along the boundary of the face coloured 2 until we reach the next vertex; etc., until we have travelled along an edge bounding a face of colour $d$. The vertex that we have reached must carry label 2; etc. Clearly, if drawn appropriately into the plane, a $d$-cactus resulting from an application of the above procedure to a minimal 
factorisation (5.3) is symmetric with respect to a rotation by $180^{\circ}$, with the centre of the rotation being the centre of the regular $2 \alpha$-gon corresponding to the unique type $B$ cycle of $\sigma_{j}$; cf. Figure 2. In what follows, we shall abbreviate this property as rotation-symmetric.

In summary, under the assumptions of claim (i), the decomposition number $N_{B_{n}}^{\text {comb }}\left(T_{1}, T_{2}, \ldots, T_{d}\right)$, respectively, if $\alpha \geq 2$, the decomposition number $N_{B_{n}}\left(T_{1}, T_{2}\right.$, $\left.\ldots, T_{d}\right)$ also, equals the number of all rotation-symmetric $d$-cacti on $2 n$ vertices in which one vertex is marked and all vertices have rotator $(1,2, \ldots, d)^{\mathrm{O}}$, with exactly $m_{k}^{(i)}$ pairs of faces of colour $i$ having $k+1$ vertices, arranged symmetrically around a central face of colour $j$ with $2 \alpha$ vertices.

Aside from the marking of one vertex, equivalent objects are counted in [12, Theorem 25]. In our language, modulo the "dualisation" described in Footnote 1 . and upon replacing $m$ by $d$, the objects which are counted in the cited theorem are $d$-cacti in which all vertices have rotator $(1,2, \ldots, d)^{\mathrm{O}}$, and which are invariant under a rotation (not necessarily by $180^{\circ}$ ). To be precise, from the proof of [12, (81)] (not given in full detail in [12]) it can be extracted that the number of $d$-cacti on $2 n$ vertices, in which all vertices have rotator $(1,2, \ldots, d)^{\mathrm{O}}$, which are invariant under a rotation by $(360 / s)^{\circ}, s$ being maximal with this property, and which have exactly $2 m_{k}^{(i)}$ faces of colour $i$ having $k+1$ vertices arranged around a central face of colour $j$ with $2 \alpha$ vertices, equals

$$
\begin{gathered}
(2 n)^{d-2} s \sum_{t}^{\prime} \mu(t / s)\left(\begin{array}{c}
2\left(n-\mathrm{rk} T_{j}\right) / t \\
2 m_{1}^{(j)} / t, 2 m_{2}^{(j)} / t, \ldots, 2 m_{n}^{(j)} / t
\end{array}\right) \\
\cdot \prod_{\substack{i=1 \\
i \neq j}}^{d} \frac{1}{2\left(n-\operatorname{rk} T_{i}\right)}\left(\begin{array}{c}
2\left(n-\operatorname{rk} T_{i}\right) / t \\
2 m_{1}^{(i)} / t, 2 m_{2}^{(i)} / t, \ldots, 2 m_{n}^{(i)} / t
\end{array}\right),
\end{gathered}
$$

where the sum extends over all $t$ with $s|t, t| 2 \alpha$, and $t \mid 2 m_{k}^{(i)}$ for all $i=1,2, \ldots, d$ and $k=1,2, \ldots, n$. Here, $\mu(\cdot)$ is the Möbius function from number theory 2 In presenting the formula in the above form, we have also used the observation that, for all $i$ (including $i=j$ !), the number of type $A$ cycles of $\sigma_{i}$ is $n-\operatorname{rk} T_{i}$.

As we said above, the $d$-cacti that we want to enumerate have one marked vertex, whereas the $d$-cacti counted by (5.9) have no marked vertex. However, given a $d$ cactus counted by (5.9), we have exactly $2 n / s$ inequivalent ways of marking a vertex. Hence, recalling that the $d$-cacti that we want to count are invariant under a rotation by $180^{\circ}$, we must multiply the expression (5.9) by $2 n / s$, and then sum the result over all even s. Since, by definition of the Möbius function, we have

$$
\sum_{2|s| t} \mu(t / s)=\sum_{s^{\prime} \mid \frac{t}{2}} \mu\left(t / 2 s^{\prime}\right)= \begin{cases}1 & \text { if } \frac{t}{2}=1 \\ 0 & \text { otherwise }\end{cases}
$$

the result of this summation is exactly the right-hand side of (5.1).

Finally, we prove claim (ii). From what we already know, in a minimal factorisation (5.3) exactly one of the factors on the right-hand side must contain a type $B$ cycle of length 1 in its (type $B$ ) disjoint cycle decomposition, $\sigma_{j}$ say. As a parabolic

\footnotetext{
${ }^{2}$ Formula (81) in [12] does not distinguish the colour or the size of the central face (that is, in the language of [12]: the colour or the degree of the central vertex). Therefore it is in fact a sum over all possible colours and sizes, represented there by the summations over $i$ and $h$, respectively.
} 
Coxeter element, a type $B$ cycle of length 1 has type $A_{1}$. Since all considerations in the proof of claim (i) are also valid for $\alpha=1$, we may use formula (5.1) with $\alpha=1$, and with $m_{1}^{(j)}$ replaced by $m_{1}^{(j)}-1$, to count the number of these factorisations, to obtain

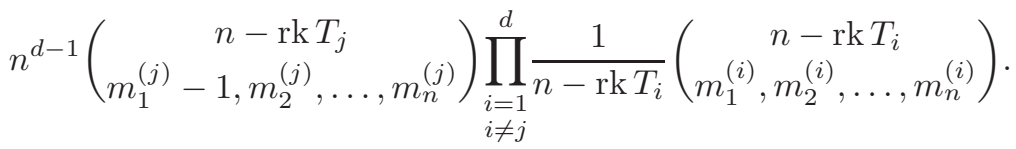

This has to be summed over $j=1,2, \ldots, d$. The result is exactly (5.2).

The proof of the theorem is now complete.

Combining the previous theorem with the summation formula of Lemma 4 we can now derive compact formulae for all type $B_{n}$ decomposition numbers.

Theorem 8. (i) Let the types $T_{1}, T_{2}, \ldots, T_{d}$ be given, where

$$
T_{i}=A_{1}^{m_{1}^{(i)}} * A_{2}^{m_{2}^{(i)}} * \cdots * A_{n}^{m_{n}^{(i)}}, \quad i=1,2, \ldots, j-1, j+1, \ldots, d,
$$

and

$$
T_{j}=B_{\alpha} * A_{1}^{m_{1}^{(j)}} * A_{2}^{m_{2}^{(j)}} * \cdots * A_{n}^{m_{n}^{(j)}},
$$

for some $\alpha \geq 1$. Then

$$
\begin{aligned}
& N_{B_{n}}^{c o m b}\left(T_{1}, T_{2}, \ldots, T_{d}\right)=n^{d-1}\left(\begin{array}{c}
n \\
\operatorname{rk} T_{1}+\operatorname{rk} T_{2}+\cdots+\operatorname{rk} T_{d}
\end{array}\right) \\
& \times\left(\begin{array}{c}
n-\operatorname{rk} T_{j} \\
m_{1}^{(j)}, m_{2}^{(j)}, \ldots, m_{n}^{(j)}
\end{array}\right) \prod_{\substack{i=1 \\
i \neq j}}^{d} \frac{1}{n-\operatorname{rk} T_{i}}\left(\begin{array}{c}
n-\operatorname{rk} T_{i} \\
m_{1}^{(i)}, m_{2}^{(i)}, \ldots, m_{n}^{(i)}
\end{array}\right),
\end{aligned}
$$

where the multinomial coefficient is defined as in Lemma 4. For $\alpha \geq 2$, the number $N_{B_{n}}\left(T_{1}, T_{2}, \ldots, T_{d}\right)$ is given by the same formula.

(ii) Let the types $T_{1}, T_{2}, \ldots, T_{d}$ be given, where

$$
T_{i}=A_{1}^{m_{1}^{(i)}} * A_{2}^{m_{2}^{(i)}} * \cdots * A_{n}^{m_{n}^{(i)}}, \quad i=1,2, \ldots, d .
$$

Then

$$
\begin{aligned}
& N_{B_{n}}^{c o m b}\left(T_{1}, T_{2}, \ldots, T_{d}\right) \\
= & n^{d}\left(\begin{array}{c}
n-1 \\
\operatorname{rk} T_{1}+\operatorname{rk} T_{2}+\cdots+\operatorname{rk} T_{d}
\end{array}\right)\left(\prod_{i=1}^{d} \frac{1}{n-\operatorname{rk} T_{i}}\left(\begin{array}{c}
n-\operatorname{rk} T_{i} \\
m_{1}^{(i)}, m_{2}^{(i)}, \ldots, m_{n}^{(i)}
\end{array}\right)\right),
\end{aligned}
$$

whereas

$$
\begin{aligned}
& =n^{d-1}\left(\begin{array}{c}
n \\
\operatorname{rk} T_{1}+\operatorname{rk} T_{2}+\cdots+\operatorname{rk} T_{d}
\end{array}\right)\left(\prod_{i=1}^{d} \frac{1}{n-\operatorname{rk} T_{i}}\left(\begin{array}{c}
n-\operatorname{rk} T_{i} \\
m_{1}^{(i)}, m_{2}^{(i)}, \ldots, m_{n}^{(i)}
\end{array}\right)\right) \\
& \times\left(n-\operatorname{rk} T_{1}-\operatorname{rk} T_{2}-\cdots-\operatorname{rk} T_{d}+\sum_{j=1}^{d} \frac{m_{1}^{(j)}\left(n-\operatorname{rk} T_{j}\right)}{m_{0}^{(j)}+1}\right),
\end{aligned}
$$

with $m_{0}^{(j)}=n-\operatorname{rk} T_{j}-\sum_{s=1}^{n} m_{s}^{(j)}$. 
(iii) All of the other decomposition numbers $N_{B_{n}}\left(T_{1}, T_{2}, \ldots, T_{d}\right)$ and $N_{B_{n}}^{\text {comb }}\left(T_{1}, T_{2}, \ldots, T_{d}\right)$ are zero.

Proof. If we write $r$ for $n-\operatorname{rk} T_{1}-\operatorname{rk} T_{2}-\cdots-\operatorname{rk} T_{d}$, then for $\Phi=B_{n}$ the relation (2.3) becomes

$$
N_{B_{n}}\left(T_{1}, T_{2}, \ldots, T_{d}\right)=\sum_{T: \mathrm{rk} T=r} N_{B_{n}}\left(T_{1}, T_{2}, \ldots, T_{d}, T\right)
$$

with the same relation holding for $N_{B_{n}}^{\text {comb }}$ in place of $N_{B_{n}}$.

In order to prove (5.10), we let $T=A_{1}^{m_{1}} * A_{2}^{m_{2}} * \cdots * A_{n}^{m_{n}}$ and use (5.1) in (5.13) to obtain

$$
\begin{aligned}
& N_{B_{n}}^{\mathrm{comb}}\left(T_{1}, T_{2}, \ldots, T_{d}\right)=\sum_{m_{1}+2 m_{2}+\cdots+n m_{n}=r} n^{d} \frac{1}{n-r}\left(\begin{array}{c}
n-r \\
m_{1}, m_{2}, \ldots, m_{n}
\end{array}\right) \\
& \cdot\left(\begin{array}{c}
n-\operatorname{rk} T_{j} \\
m_{1}^{(j)}, m_{2}^{(j)}, \ldots, m_{n}^{(j)}
\end{array}\right) \prod_{\substack{i=1 \\
i \neq j}}^{d} \frac{1}{n-\operatorname{rk} T_{i}}\left(\begin{array}{c}
n-\operatorname{rk} T_{i} \\
m_{1}^{(i)}, m_{2}^{(i)}, \ldots, m_{n}^{(i)}
\end{array}\right) .
\end{aligned}
$$

If we use (3.4) with $M=n-r$, we arrive at our claim after little simplification.

In order to prove (5.11), we let $T=B_{\alpha} * A_{1}^{m_{1}} * A_{2}^{m_{2}} * \cdots * A_{n}^{m_{n}}$ in (5.13). The important point to be observed here is that, in contrast to the previous argument, in the present case $T$ must have a factor $B_{\alpha}$. Subsequently, use of (5.1) in (5.13) yields

$$
\begin{array}{r}
N_{B_{n}}^{\text {comb }}\left(T_{1}, T_{2}, \ldots, T_{d}\right)=\sum_{\alpha=1}^{n} \sum_{m_{1}+2 m_{2}+\cdots+n m_{n}=r-\alpha} n^{d}\left(\begin{array}{c}
n-r \\
m_{1}, m_{2}, \ldots, m_{n}
\end{array}\right) \\
\cdot \prod_{i=1}^{d} \frac{1}{n-\operatorname{rk} T_{i}}\left(\begin{array}{c}
n-\operatorname{rk} T_{i} \\
m_{1}^{(i)}, m_{2}^{(i)}, \ldots, m_{n}^{(i)}
\end{array}\right) .
\end{array}
$$

Now we use (3.4) with $r$ replaced by $r-\alpha$ and $M=n-r$, and subsequently the elementary summation formula

$$
\sum_{\alpha=1}^{n}\left(\begin{array}{c}
n-\alpha-1 \\
r-\alpha
\end{array}\right)=\sum_{\alpha=1}^{n}\left(\begin{array}{c}
n-\alpha-1 \\
n-r-1
\end{array}\right)=\left(\begin{array}{l}
n-1 \\
n-r
\end{array}\right)=\left(\begin{array}{l}
n-1 \\
r-1
\end{array}\right)
$$

Then, after little rewriting, we arrive at our claim.

To establish (5.12), we must recall that the group-theoretic type $A_{1}$ does not distinguish between a type $A$ cycle $((i, j))=(i, j)(\bar{i}, \bar{j})$ and a type $B$ cycle $[i]=$ $(i, \bar{i})$. Hence, to obtain $N_{B_{n}}\left(T_{1}, T_{2}, \ldots, T_{d}\right)$ in the case that no $T_{i}$ contains a $B_{\alpha}$ for $\alpha \geq 2$, we must add the expression (5.11) and the expressions (5.10) with $m_{1}^{(j)}$ replaced by $m_{1}^{(j)}-1$ over $j=1,2, \ldots, d$. As is not difficult to see, this sum is indeed equal to (5.12).

\section{DeComposition NUmBers For type $D$}

In this section we compute the decomposition numbers for type $D_{n}$. Theorem 9 gives the formulae for the full rank decomposition numbers, while Theorem 10 presents the implied formulae for the decomposition numbers of arbitrary rank. To our knowledge, these are new results, which did not appear earlier in the literature on map enumeration or on the connection coefficients in the symmetric group or 
other Coxeter groups. Nevertheless, the proof of Theorem 9 is entirely in the spirit of the fundamental paper [23, in that the problem of counting factorisations is translated into a problem of map enumeration, which is then solved by a generating function approach that requires the use of the Lagrange-Good formula for coefficient extraction.

We begin with the result concerning the full rank decomposition numbers in type $D_{n}$. While reading the statement of the theorem below, the reader should again recall from Section 2 the distinction between group-theoretic and combinatorial decomposition numbers.

Theorem 9. (i) If $T_{1}, T_{2}, \ldots, T_{d}$ are types with $\operatorname{rk} T_{1}+\operatorname{rk} T_{2}+\cdots+\operatorname{rk} T_{d}=n$, where

$$
T_{i}=A_{1}^{m_{1}^{(i)}} * A_{2}^{m_{2}^{(i)}} * \cdots * A_{n}^{m_{n}^{(i)}}, \quad i=1,2, \ldots, j-1, j+1, \ldots, d
$$

and

$$
T_{j}=D_{\alpha} * A_{1}^{m_{1}^{(j)}} * A_{2}^{m_{2}^{(j)}} * \cdots * A_{n}^{m_{n}^{(j)}}
$$

for some $\alpha \geq 2$, then

$$
\begin{aligned}
N_{D_{n}}^{c o m b}\left(T_{1}, T_{2}, \ldots, T_{d}\right)=(n-1)^{d-1} & \left(\begin{array}{c}
n-\mathrm{rk} T_{j} \\
m_{1}^{(j)}, m_{2}^{(j)}, \ldots, m_{n}^{(j)}
\end{array}\right) \\
\times & \prod_{\substack{i=1 \\
i \neq j}}^{d} \frac{1}{n-\operatorname{rk} T_{i}-1}\left(\begin{array}{c}
n-\operatorname{rk} T_{i}-1 \\
m_{1}^{(i)}, m_{2}^{(i)}, \ldots, m_{n}^{(i)}
\end{array}\right),
\end{aligned}
$$

where the multinomial coefficient is defined as in Lemma 4. For $\alpha \geq 4$, the number $N_{D_{n}}\left(T_{1}, T_{2}, \ldots, T_{d}\right)$ is given by the same formula.

(ii) If $T_{1}, T_{2}, \ldots, T_{d}$ are types with $\operatorname{rk} T_{1}+\operatorname{rk} T_{2}+\cdots+\operatorname{rk} T_{d}=n$, where

$$
T_{i}=A_{1}^{m_{1}^{(i)}} * A_{2}^{m_{2}^{(i)}} * \cdots * A_{n}^{m_{n}^{(i)}}, \quad i=1,2, \ldots, d
$$

then

$$
\begin{array}{r}
=(n-1)^{d-1}\left(2 \sum_{j=1}^{d}\left(\begin{array}{c}
n-\mathrm{rk} T_{j} \\
m_{1}^{(j)}, m_{2}^{(j)}, \ldots, m_{n}^{(j)}
\end{array}\right) \prod_{\substack{i=1 \\
i \neq j}}^{d} \frac{1}{n-\operatorname{rk} T_{i}-1}\left(\begin{array}{c}
n-\operatorname{rk} T_{i}-1 \\
m_{1}^{(i)}, m_{2}^{(i)}, \ldots, m_{n}^{(i)}
\end{array}\right)\right. \\
\left.\quad-2(d-1)(n-1) \prod_{i=1}^{d} \frac{1}{n-\operatorname{rk} T_{i}-1}\left(\begin{array}{c}
n-\operatorname{rk} T_{i}-1 \\
m_{1}^{(i)}, m_{2}^{(i)}, \ldots, m_{n}^{(i)}
\end{array}\right)\right),
\end{array}
$$


while

(6.3)

$$
\begin{aligned}
& N_{D_{n}}\left(T_{1}, T_{2}, \ldots, T_{d}\right) \\
&=(n-1)^{d-1}\left(\sum _ { j = 1 } ^ { d } ( \prod _ { \substack { i = 1 \\
i \neq j } } ^ { d } \frac { 1 } { n - \operatorname { r k } T _ { i } - 1 } ( \begin{array} { c } 
{ n - \operatorname { r k } T _ { i } - 1 } \\
{ m _ { 1 } ^ { ( i ) } , m _ { 2 } ^ { ( i ) } , \ldots , m _ { n } ^ { ( i ) } }
\end{array} ) ) \left(2\left(\begin{array}{c}
n-\operatorname{rk} T_{j} \\
m_{1}^{(j)}, m_{2}^{(j)}, \ldots, m_{n}^{(j)}
\end{array}\right)\right.\right. \\
&\left.+\left(m_{1}^{(j)}, m_{2}^{(j)}, m_{3}^{(j)}-1, m_{4}^{(j)}, \ldots, m_{n}^{(j)}\right)+\left(\begin{array}{c}
n-\operatorname{rk} T_{j} \\
m_{1}^{(j)}-2, m_{2}^{(j)}, \ldots, m_{n}^{(j)}
\end{array}\right)\right) \\
&\left.-2(d-1)(n-1) \prod_{i=1}^{d} \frac{1}{n-\operatorname{rk} T_{i}-1}\left(\begin{array}{c}
n-\operatorname{rk} T_{i}-1 \\
m_{1}^{(i)}, m_{2}^{(i)}, \ldots, m_{n}^{(i)}
\end{array}\right)\right) .
\end{aligned}
$$

(iii) All of the other decomposition numbers $N_{D_{n}}\left(T_{1}, T_{2}, \ldots, T_{d}\right)$ and $N_{D_{n}}^{\text {comb }}\left(T_{1}, T_{2}, \ldots, T_{d}\right)$ with $\operatorname{rk} T_{1}+\operatorname{rk} T_{2}+\cdots+\operatorname{rk} T_{d}=n$ are zero.

Remark. These formulae must be correctly interpreted when $T_{i}$ contains no $D_{\alpha}$ and $\operatorname{rk} T_{i}=n-1$. In that case, because of $n-1=\operatorname{rk} T_{i}=m_{1}^{(i)}+2 m_{2}^{(i)}+\cdots+n m_{n}^{(i)}$, there must be an $\ell, 1 \leq \ell \leq n-1$, with $m_{\ell}^{(i)} \geq 1$. We then interpret the term

as

$$
\frac{1}{n-\operatorname{rk} T_{i}-1}\left(\begin{array}{c}
n-\operatorname{rk} T_{i}-1 \\
m_{1}^{(i)}, m_{2}^{(i)}, \ldots, m_{n}^{(i)}
\end{array}\right)
$$

$$
\frac{1}{n-\operatorname{rk} T_{i}-1}\left(\begin{array}{c}
n-\operatorname{rk} T_{i}-1 \\
m_{1}^{(i)}, m_{2}^{(i)}, \ldots, m_{n}^{(i)}
\end{array}\right)=\frac{1}{m_{\ell}^{(i)}}\left(\begin{array}{c}
n-\operatorname{rk} T_{i}-2 \\
m_{1}^{(i)}, \ldots, m_{\ell}^{(i)}-1, \ldots, m_{n}^{(i)}
\end{array}\right),
$$

where the multinomial coefficient is zero whenever

$$
-1=n-\operatorname{rk} T_{i}-2<m_{1}^{(i)}+\cdots+\left(m_{\ell}^{(i)}-1\right)+\cdots+m_{n}^{(i)},
$$

except when all of $m_{1}^{(i)}, \ldots, m_{\ell}^{(i)}-1, \ldots, m_{n}^{(i)}$ are zero. Explicitly, one must read

$$
\frac{1}{n-\operatorname{rk} T_{i}-1}\left(\begin{array}{c}
n-\operatorname{rk} T_{i}-1 \\
m_{1}^{(i)}, m_{2}^{(i)}, \ldots, m_{n}^{(i)}
\end{array}\right)=0
$$

if $\operatorname{rk} T_{i}=n-1$ but $T_{i} \neq A_{n-1}$, and

if $T_{i}=A_{n-1}$.

$$
\frac{1}{n-\operatorname{rk} T_{i}-1}\left(\begin{array}{c}
n-\operatorname{rk} T_{i}-1 \\
m_{1}^{(i)}, m_{2}^{(i)}, \ldots, m_{n}^{(i)}
\end{array}\right)=1
$$

Proof of Theorem 9, Determining the decomposition number

$$
N_{D_{n}}\left(T_{1}, T_{2}, \ldots, T_{d}\right)=N_{D_{n}}\left(T_{d}, \ldots, T_{2}, T_{1}\right)
$$

(recall (2.2)), respectively

$$
N_{D_{n}}^{\mathrm{comb}}\left(T_{1}, T_{2}, \ldots, T_{d}\right)=N_{D_{n}}^{\mathrm{comb}}\left(T_{d}, \ldots, T_{2}, T_{1}\right),
$$

amounts to counting all possible factorisations

$$
(1,2, \ldots, n-1, \overline{1}, \overline{2}, \ldots, \overline{n-1})(n, \bar{n})=\sigma_{d} \cdots \sigma_{2} \sigma_{1},
$$

where $\sigma_{i}$ has type $T_{i}$ as a parabolic Coxeter element, respectively has combinatorial type $T_{i}$. Here also, the factorisation (6.4) is minimal in the sense that

$$
n=\ell_{T}((1,2, \ldots, n-1, \overline{1}, \overline{2}, \ldots, \overline{n-1})(n, \bar{n}))=\ell_{T}\left(\sigma_{1}\right)+\ell_{T}\left(\sigma_{2}\right)+\cdots+\ell_{T}\left(\sigma_{d}\right),
$$


since $\ell_{T}\left(\sigma_{i}\right)=\operatorname{rk} T_{i}$, and since, by our assumption, the sum of the ranks of the $T_{i}$ 's equals $n$.

We first prove claim (iii). Let us assume, for contradiction, that there is a minimal factorisation (6.4), in which, altogether, we find at least two type $B$ cycles of length $\geq 2$ in the (type $B$ ) disjoint cycle decompositions of the $\sigma_{i}$ 's. It can then be shown by arguments similar to those in the proof of claim (iii) in Theorem 7 that this leads to a contradiction. Hence, "at worst," we may find a type $B$ cycle of length $1,(a, \bar{a})$ say, and another type $B$ cycle, $\kappa$ say. Both of them must be contained in the disjoint cycle decomposition of one of the $\sigma_{i}$ 's since all the $\sigma_{i}$ 's are elements of $W\left(D_{n}\right)$. Given that $\kappa$ has length $\alpha-1$, the product of both, $(a, \bar{a}) \kappa$, is of combinatorial type $D_{\alpha}, \alpha \geq 2$, whereas, as a parabolic Coxeter element, it is of type $D_{\alpha}$ only if $\alpha \geq 4$. If $\alpha=3$, then it is a parabolic Coxeter element of type $A_{3}$, and if $\alpha=2$ it is of type $A_{1}^{2}$. Thus, we are actually in the cases to which claims (i) and (ii) apply.

To prove claim (i), we continue this line of argument. By a variation of the conjugation argument (5.4) $-(5.6)$, we may assume that these two type $B$ cycles are contained in $\sigma_{d}, \sigma_{d}=(a, \bar{a}) \kappa \sigma_{d}^{\prime}$ say, where, as above, $(a, \bar{a})$ is the type $B$ cycle of length 1 and $\kappa$ is the other type $B$ cycle, and where $\sigma_{d}^{\prime}$ is free of type $B$ cycles. In that case, (6.4) takes the form

$$
c=(1,2, \ldots, n-1, \overline{1}, \overline{2}, \ldots, \overline{n-1})(n, \bar{n})=(a, \bar{a}) \kappa \sigma_{d}^{\prime} \cdots \sigma_{1} .
$$

If $a \neq n, \kappa \neq(n, \bar{n})$, and if $\kappa$ does not fix $n$, then $(a, \bar{a}) \kappa \mathbb{E}_{T} c$, a contradiction. Likewise, if $a \neq n, \kappa=\left[b_{1}, b_{2}, \ldots, b_{k}\right]$ with $n \notin\left\{b_{1}, b_{2}, \ldots, b_{k}\right\}$, then $(a, \bar{a}) \kappa \mathbb{Z}_{T}$ $[1,2, \ldots, n-1]$, again a contradiction. Hence, we may assume that $a=n$, whence $(a, \bar{a}) \kappa=\kappa(n, \bar{n})$ forms a parabolic Coxeter element of type $D_{\alpha}$, given that $\kappa$ has length $\alpha-1$. We are then in the position to determine all possible factorisations of the form 6.5), which reduces to

$$
(1,2, \ldots, n-1, \overline{1}, \overline{2}, \ldots, \overline{n-1})=[1,2, \ldots, n-1]=\kappa \sigma_{d}^{\prime} \cdots \sigma_{1} .
$$

This is now a minimal type $B$ factorisation of the form (5.3) with $n$ replaced by $n-1$. We may therefore use formula (5.1) with $n$ replaced by $n-1$ and with $\operatorname{rk} T_{j}$ replaced by $\operatorname{rk} T_{j}-1$. These substitutions lead exactly to (6.1).

Finally, we turn to claim (ii). First we discuss two degenerate cases which come from the identifications $D_{3} \sim A_{3}$, respectively $D_{2} \sim A_{1}^{2}$, and which only occur for $N_{D_{n}}\left(T_{1}, T_{2}, \ldots, T_{d}\right)$ (but not for the combinatorial decomposition numbers $\left.N_{D_{n}}^{\text {comb }}\left(T_{1}, T_{2}, \ldots, T_{d}\right)\right)$. It may happen that one of the factors in (6.4), let us say, without loss of generality, $\sigma_{d}$, contains a type $B$ cycle of length 1 and one of length 2 in its disjoint cycle decomposition; that is, $\sigma_{d}$ may contain

$$
(n, \bar{n})[a, b]=(n, \bar{n})(a, b, \bar{a}, \bar{b})=[a, b][b, n][b, \bar{n}] .
$$

As a parabolic Coxeter element, this is of type $A_{3}$. By the reduction (6.5)-(6.6), we may count the number of these possibilities by formula (5.1) with $n$ replaced by $n-1, \operatorname{rk} T_{j}$ replaced by $\operatorname{rk} T_{j}-1$, and $m_{3}^{(j)}$ replaced by $m_{3}^{(j)}-1$. This explains the second term in the factor in large parentheses on the right-hand side of (6.3). On the other hand, it may happen that one of the factors in (6.4), let us say again, without loss of generality, $\sigma_{d}$, contains two type $B$ cycles of length 1 in its disjoint cycle decomposition; that is, $\sigma_{d}$ may contain $(n, \bar{n})(a, \bar{a})$. As a parabolic Coxeter element, this is of type $A_{1}^{2}$. By the reduction (6.5) of these possibilities by formula (5.1) with $n$ replaced by $n-1, \operatorname{rk} T_{j}$ replaced by 


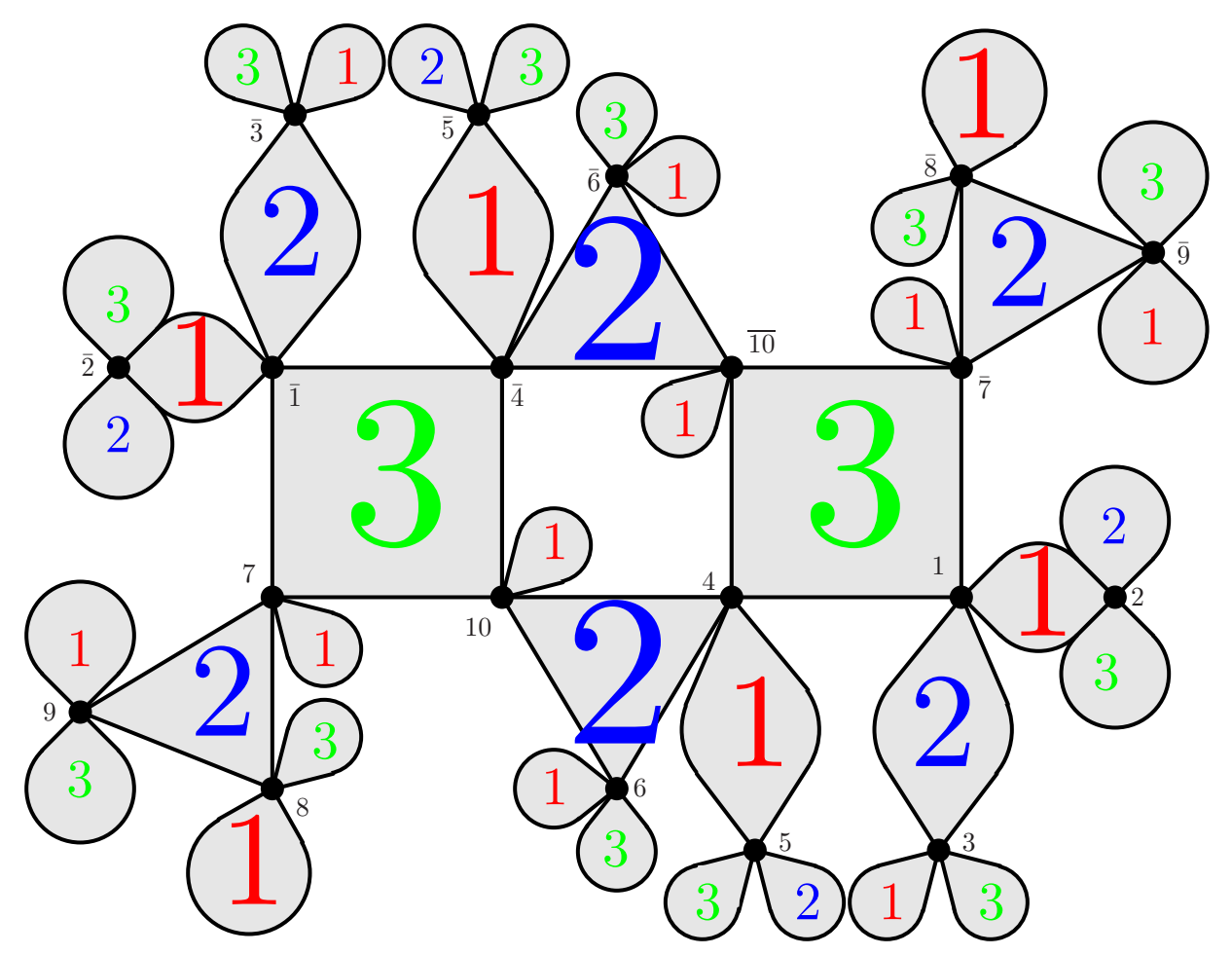

Figure 3. The 3-atoll corresponding to the factorisation (6.7)

$\operatorname{rk} T_{j}-1$, and $m_{1}^{(j)}$ replaced by $m_{1}^{(j)}-2$. This explains the third term in the factor in large parentheses on the right-hand side of (6.3).

From now on we may assume that none of the $\sigma_{i}$ 's contains a type $B$ cycle in its (type $B$ ) disjoint cycle decomposition. To determine the number of minimal factorisations (6.4) in this case, we again construct a bijection between these factorisations and certain maps. In what follows, we will still use the concept of a rotator, introduced in the proof of Theorem 7 We again apply the procedure described in that proof. That is, for each (ordinary) cycle $\left(a_{1}, a_{2}, \ldots, a_{k}\right)$ of $\sigma_{i}$, we create a $k$-gon coloured $i$, label its vertices $a_{1}, a_{2}, \ldots, a_{k}$ in clockwise order, and glue these polygons into a map by identifying equally labelled vertices such that the rotator of each vertex is $(1,2, \ldots, d)$. However, this map can be embedded in the plane only if we allow the creation of an inner face corresponding to the cycle $(n, \bar{n})$ on the left-hand side of (6.4) (the outer face corresponding to the large cycle $(1,2, \ldots, n-1, \overline{1}, \overline{2}, \ldots, \overline{n-1})$. Moreover, this inner face must be bounded by $2 d$ edges. We call such a map, in which all faces except the outer face and an inner face intersect only in vertices and are coloured with colours from $\{1,2, \ldots, d\}$, and in which the inner face is bounded by $2 d$ edges, a $d$-atoll. For example, if we take $n=10$ and $d=3$, and consider the factorisation

$$
(1,2, \ldots, 9, \overline{1}, \overline{2}, \ldots, \overline{9})(10, \overline{10})=\sigma_{3} \sigma_{2} \sigma_{1},
$$

where $\sigma_{3}=((1,4, \overline{10}, \overline{7})), \sigma_{2}=((1,3))((4,6,10))((7,8,9))$, and $\sigma_{1}=((1,2))((4,5))$, and apply this procedure, we obtain the 3 -atoll in Figure 3 In the figure, the faces corresponding to cycles are shaded. As in Figures 1 and 2 the outer face is not 


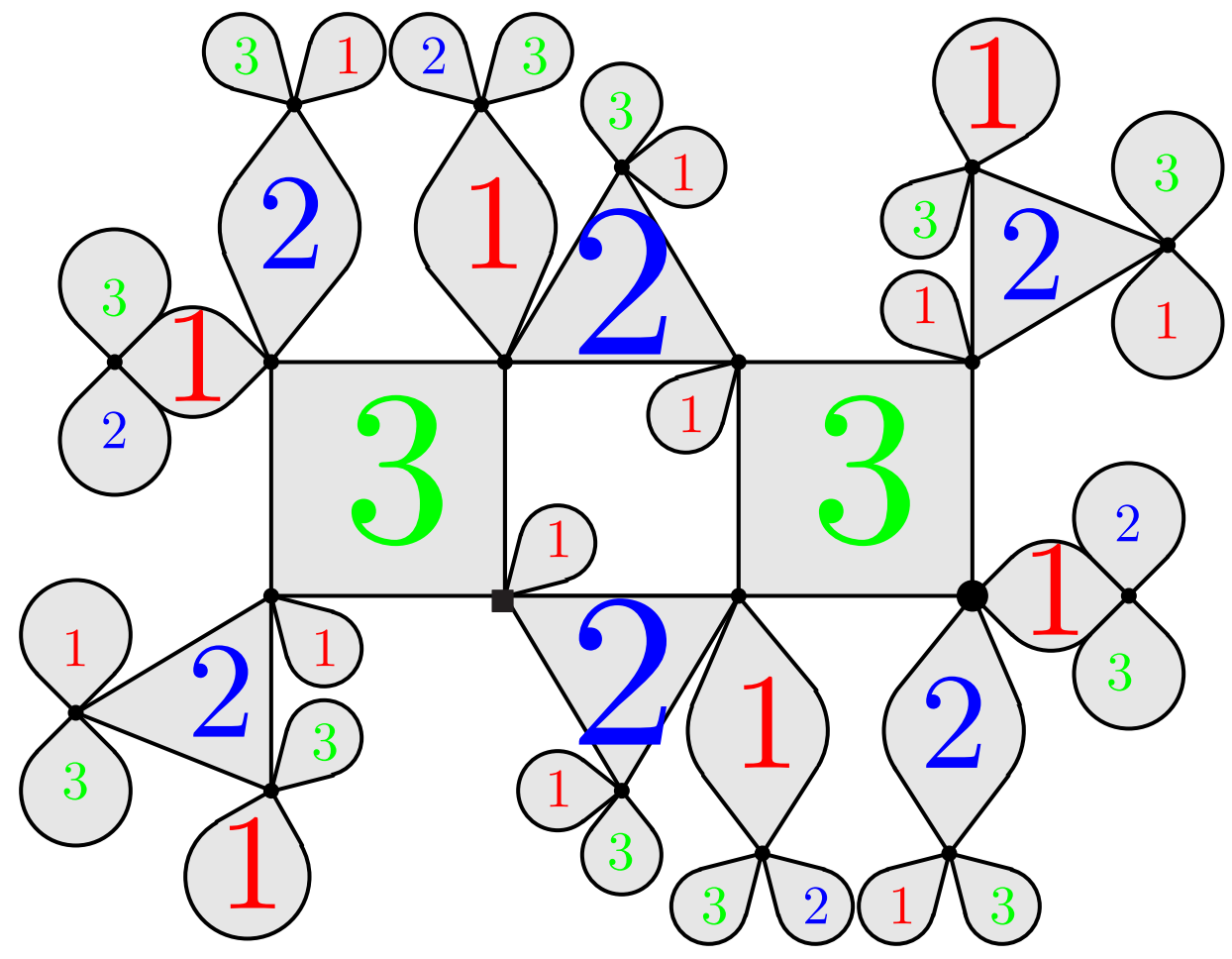

FiguRE 4. A rotation-symmetric 3-atoll with two marked vertices

shaded. Here, there is in addition an inner face which is not shaded, the face formed by the vertices $4,10, \overline{4}, \overline{10}$. Again, the colours of the shaded faces are indicated by the numbers 1,2 , respectively 3 , placed in the centre of the faces.

Unsurprisingly, the fact that the result of the procedure can be realised as a $d$-atoll follows again from Euler's formula. More precisely, the number of faces corresponding to the polygons is $2 \sum_{i=1}^{d} \sum_{k=0}^{n} m_{k}^{(i)}$, the number of edges is $2 \sum_{i=1}^{d} \sum_{k=0}^{n} m_{k}^{(i)}(k+1)$, and the number of vertices is $2 n$. Hence, if we include the outer face and the inner face, the number of vertices minus the number of edges plus the number of faces is

$$
\begin{aligned}
2 n-2 \sum_{i=1}^{d} \sum_{k=0}^{n} m_{k}^{(i)}(k+1)+ & 2 \sum_{i=1}^{d} \sum_{k=0}^{n} m_{k}^{(i)}+2=2 n+2-2 \sum_{i=1}^{d} \sum_{k=0}^{n} k \cdot m_{k}^{(i)} \\
& =2 n+2-2 \operatorname{rk} T_{1}-2 \operatorname{rk} T_{2}-\cdots-2 \operatorname{rk} T_{d} \\
& =2,
\end{aligned}
$$

according to our assumption concerning the sum of the ranks of types $T_{i}$.

Again, we may further simplify this geometric representation of a minimal factorisation (6.4) by deleting all vertex labels, marking the vertex which had label 1 with $\bullet$ and marking the vertex that had label $n$ with $\mathbf{\square}$. If this simplification is applied to the 3-atoll in Figure 3, we obtain the 3-atoll in Figure 4 . Clearly, if drawn appropriately into the plane, a $d$-atoll resulting from an application of the above procedure to a minimal factorisation (6.4) is symmetric with respect to a 
rotation by $180^{\circ}$, the centre of the rotation being the centre of the inner face; cf. Figure 4. As earlier, we shall abbreviate this property as rotation-symmetric. In fact, there is not much freedom for the choice of the vertex marked by $\boldsymbol{\square}$ once a vertex has been marked by $\bullet$. Clearly, if we run through the vertex labelling process described in the proof of Theorem [7, labelling as 1 the vertex which is marked by •, we shall reconstruct the labels $1,2, \ldots, n-1, \overline{1}, \overline{2}, \ldots, \overline{n-1}$. This leaves only 2 vertices incident to the inner face unlabelled, one of which will have to carry the mark

In summary, under the assumptions of claim (ii), the number of minimal factorisations (6.4), in which none of the $\sigma_{i}$ 's contains a type $B$ cycle in its disjoint cycle decomposition, equals twice the number of all rotation-symmetric $d$-atolls on $2 n$ vertices, in which one vertex is marked by $\bullet$, all vertices have rotator $(1,2, \ldots, d)^{\mathrm{O}}$, and with exactly $m_{k}^{(i)}$ pairs of faces of colour $i$ having $k+1$ vertices, arranged symmetrically around the inner face (which is not coloured). Let us denote the number of these $d$-atolls by $N_{D_{n}}^{\prime}\left(T_{1}, T_{2}, \ldots, T_{d}\right)$.

We must now enumerate these $d$-atolls. First of all, introducing a figure of speech, we shall refer to coloured faces of a $d$-atoll which share an edge with the inner face but not with the outer face as faces "inside the $d$-atoll," and all others as faces "outside the $d$-atoll." For example, in Figure 4 we find two faces inside the 3-atoll, namely the two loop faces attached to the vertices labelled 10, respectively $\overline{10}$, in Figure 3 . Since, in a $d$-atoll, the inner face is bounded by exactly $2 d$ edges, inside the $d$-atoll, we find only coloured faces containing exactly one vertex. Next, we travel counter-clockwise around the inner face and record the coloured faces sharing an edge with both the inner and outer faces. Thus we obtain a list of the form

$$
F_{1}, F_{2}, \ldots, F_{\ell}, F_{\ell+1}, \ldots, F_{2 \ell}
$$

where, except possibly for the marking, $F_{h+\ell}$ is an identical copy of $F_{h}, h=$ $1,2, \ldots, \ell$. In Figure 4 , this list contains four faces, $\tilde{F}_{1}, \tilde{F}_{2}, \tilde{F}_{3}, \tilde{F}_{4}$, where $\tilde{F}_{1}$ and $\tilde{F}_{3}$ are the two quadrangles of colour 3 , and where $\tilde{F}_{2}$ and $\tilde{F}_{4}$ are the two triangles of colour 2 connecting the two quadrangles.

Continuing the general argument, let the colour of $F_{h}$ be $i_{h}$. Inside the $d$-atoll, because of the rotator condition, there must be $\left\{i_{h+1}-i_{h}-1\right\}_{d}$ faces (containing just one vertex) incident to the common vertex of $F_{h}$ and $F_{h+1}$ coloured $\left\{i_{h}+\right.$ $1\}_{d}, \ldots,\left\{i_{h+1}-1\right\}_{d}$, where, by definition,

$$
\{x\}_{d}:= \begin{cases}x, & \text { if } 0 \leq x \leq d, \\ x+d, & \text { if } x<0 \\ x-d, & \text { if } x>d,\end{cases}
$$

and where $i_{h+\ell}=i_{h}, h=1,2, \ldots, \ell$. Here, if $\left\{i_{h}+1\right\}_{d}>\left\{i_{h+1}-1\right\}_{d}$, the sequence of colours $\left\{i_{h}+1\right\}_{d}, \ldots,\left\{i_{h+1}-1\right\}_{d}$ must be interpreted "cyclically," that is, as $\left\{i_{h}+1\right\}_{d},\left\{i_{h}+1\right\}_{d}+1, \ldots, d, 1,2, \ldots,\left\{i_{h+1}-1\right\}_{d}$. As we observed above, the number of edges bounding the inner face is $2 d$. On the other hand, using the notation just introduced, this number also equals

$$
2 \sum_{h=1}^{\ell}\left\{i_{h+1}-i_{h}\right\}_{d}=2 \sum_{h=1}^{\ell}\left(\left(i_{h+1}-i_{h}\right)+d \cdot \chi\left(i_{h+1}<i_{h}\right)\right)=2 d \sum_{h=1}^{\ell} \chi\left(i_{h+1}<i_{h}\right) .
$$


Hence, there is precisely one $h$ for which $i_{h+1}<i_{h}$. Without loss of generality, we may assume that $h=\ell$, so that $i_{1}<i_{2}<\cdots<i_{\ell}$.

The ascending colouring of the faces $F_{1}, F_{2}, \ldots, F_{\ell}$ breaks the (rotation) symmetry of the $d$-atoll. Therefore, we may first enumerate $d$-atolls without any marking, and multiply the result by the number of all possible markings, which is $n-1$. More precisely, let $N_{D_{n}}^{\prime \prime}\left(T_{1}, T_{2}, \ldots, T_{d}\right)$ denote the number of all rotation-symmetric $d$ atolls on $2 n$ vertices, in which all vertices have rotator $(1,2, \ldots, d)^{\mathrm{O}}$, and with exactly $m_{k}^{(i)}$ pairs of faces of colour $i$ having $k+1$ vertices, arranged symmetrically around the inner face (which is not coloured). Then,

$$
\begin{aligned}
N_{D_{n}}\left(T_{1}, T_{2}, \ldots, T_{d}\right) & =2 N_{D_{n}}^{\prime}\left(T_{1}, T_{2}, \ldots, T_{d}\right) \\
& =2(n-1) N_{D_{n}}^{\prime \prime}\left(T_{1}, T_{2}, \ldots, T_{d}\right) .
\end{aligned}
$$

We use a generating function approach to determine $N_{D_{n}}^{\prime \prime}\left(T_{1}, T_{2}, \ldots, T_{d}\right)$, which requires a combinatorial decomposition of our objects. Let $G(\mathbf{z})$ be the generating function

$$
G(\mathbf{z})=\sum_{A \in \mathcal{A}} w(A)
$$

where $\mathcal{A}$ is the set of all rotation-symmetric $d$-atolls, in which all vertices have rotator $(1,2, \ldots, d)^{\mathrm{O}}$, and where

$$
w(A)=\prod_{i=1}^{d} z_{i}^{\frac{1}{2} \#(\text { faces of } A \text { with colour } i)} \prod_{i=1}^{d} \prod_{k=1}^{\infty} p_{i, k}^{\frac{1}{2} \# \text { (faces of } A \text { with colour } i \text { and } k \text { vertices) }} .
$$

Here, $\mathbf{z}=\left(z_{1}, z_{2}, \ldots, z_{d}\right)$, with the $z_{i}^{\prime}$ 's, $i=1,2, \ldots, d$, and the $p_{i, k}$ 's, $i=1,2, \ldots, d$, $k=1,2, \ldots$, being indeterminates. Clearly, in view of the bijection between minimal factorisations (6.4) and $d$-atolls described earlier, and by (6.9), we have

$$
N_{D_{n}}\left(T_{1}, T_{2}, \ldots, T_{d}\right)=2(n-1)\left\langle\mathbf{z}^{\mathbf{c}} \prod_{i=1}^{d} \prod_{k=0}^{n} p_{i, k+1}^{m_{k}^{(i)}}\right\rangle G(\mathbf{z}),
$$

where $\mathbf{c}=\left(c_{1}, c_{2}, \ldots, c_{d}\right)$, with $c_{i}$ equal to the number of type $A$ cycles of $\sigma_{i}$; that is, $c_{i}=\sum_{k=0}^{n} m_{k}^{(i)}, i=1,2, \ldots, d$. Here, and in the sequel, we use the multi-index notation introduced at the beginning of Section 3 For later use, we observe that, for all $i, c_{i}$ is related to $\operatorname{rk} T_{i}$ via

$$
c_{i}=n-\operatorname{rk} T_{i} .
$$

Now, let $A$ be a $d$-atoll in $\mathcal{A}$ such that the faces which share an edge with both the inner and outer faces are

$$
F_{1}, F_{2}, \ldots, F_{\ell}, F_{\ell+1}, \ldots, F_{2 \ell},
$$

where $F_{h+\ell}$ is an identical copy of $F_{h}$, where the colour of $F_{h}$ is $i_{h}, h=1,2, \ldots, \ell$, and with $i_{1}<i_{2}<\cdots<i_{\ell}$. We decompose $A$ by separating from each other the polygons which touch in vertices of the inner face. The decomposition in the case of our example in Figure 4 is shown in Figure 5 , Ignoring identical copies which 


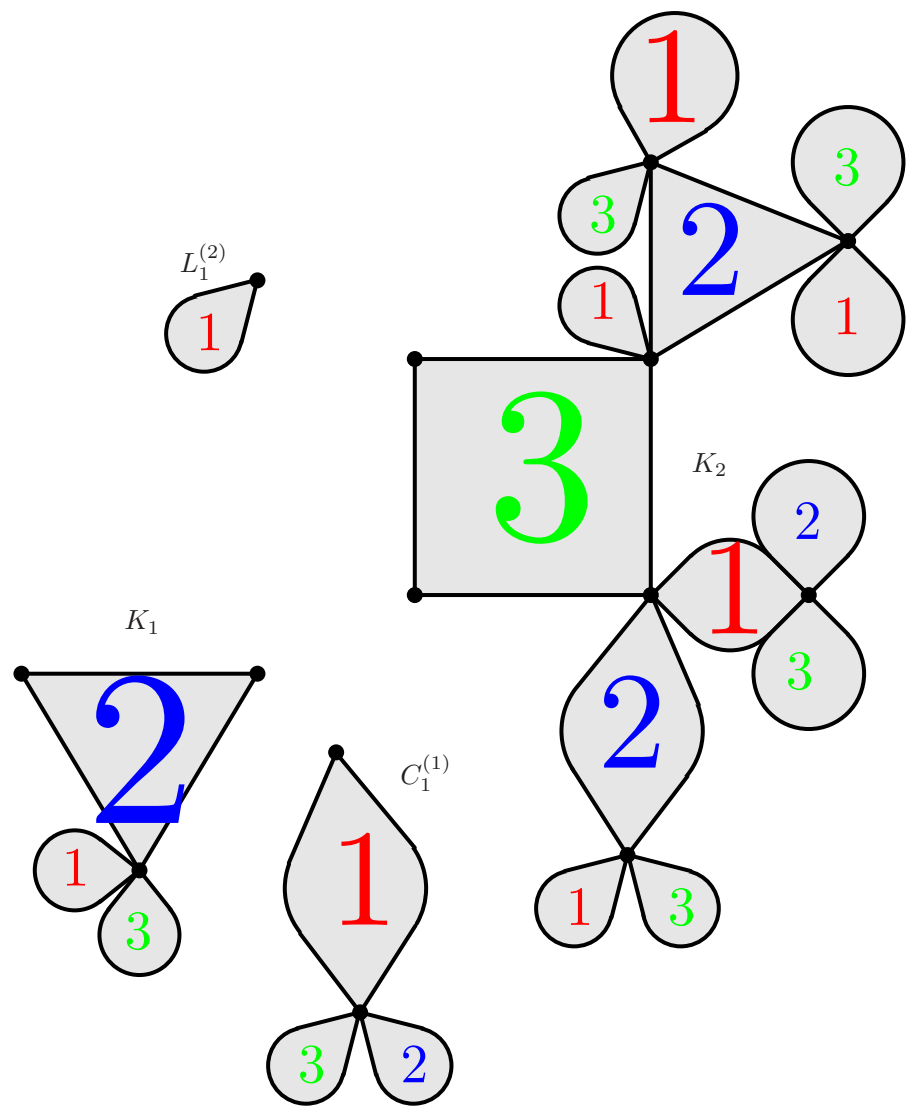

Figure 5. The decomposition of the 3-atoll in Figure 4

are there due to the rotation symmetry, we obtain a list

$$
\begin{gathered}
K_{1}, L_{i_{1}+1}^{(1)}, \ldots, L_{i_{2}-1}^{(1)}, C_{i_{2}+1}^{(1)}, \ldots, C_{d}^{(1)}, C_{1}^{(1)}, \ldots, C_{i_{1}-1}^{(1)} \\
K_{2}, L_{i_{2}+1}^{(2)}, \ldots, L_{i_{3}-1}^{(2)}, C_{i_{3}+1}^{(2)}, \ldots, C_{d}^{(2)}, C_{1}^{(2)}, \ldots, C_{i_{2}-1}^{(2)}, \ldots \\
K_{\ell}, L_{i_{\ell}+1}^{(\ell)}, \ldots, L_{d}^{(\ell)}, L_{1}^{(\ell)}, \ldots, L_{i_{1}-1}^{(\ell)}, C_{i_{1}+1}^{(\ell)}, \ldots, C_{i_{\ell}-1}^{(\ell)},
\end{gathered}
$$

where $K_{h}$ is the $d$-cactus containing the face $F_{h}$, and, hence, a $d$-cactus in which all but two neighbouring vertices have rotator $(1,2, \ldots, d)^{\mathrm{O}}$, the latter two vertices being incident to just one face, which is of colour $i_{h}$, where $L_{j}^{(h)}$ is a face of colour $j$ with just one vertex, and where $C_{j}^{(h)}$ is a $d$-cactus in which all but one vertex have rotator $(1,2, \ldots, d)^{\mathrm{O}}$, the distinguished vertex being incident to just one face, which is of colour $j, h=1,2, \ldots, \ell$ and $j=1,2, \ldots, d$. With this notation, our example in Figure 5 is one in which $\ell=2, i_{1}=2, i_{2}=3$.

The $d$-cacti $K_{h}$ can be further decomposed. Namely, assuming that the face $F_{h}$ is a $k$-gon (of colour $i_{h}$ ), let $C_{1}, C_{2}, \ldots, C_{k-2}$ be the $d$-cacti incident to this $k$-gon, read in clockwise order, starting with the $d$-cactus to the left of the two distinguished vertices. Figure [6 illustrates this further decomposition of the $d$ cactus $K_{2}$ from Figure 5 . After removal of $F_{h}$, we are left with the ordered collection 


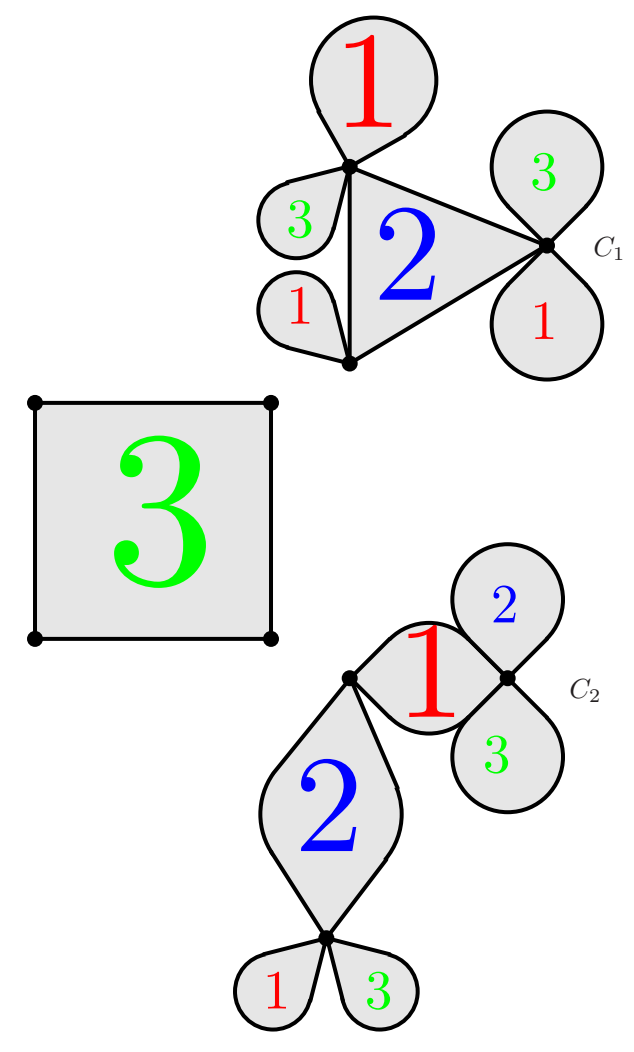

Figure 6. The decomposition of $K_{2}$ in Figure 5

$C_{1}, C_{2}, \ldots, C_{k-2}$ of $d$-cacti, each having the property that the rotator of all but one vertex is $(1,2, \ldots, d)^{\mathrm{O}}$, the exceptional vertex having rotator $\left(1, \ldots, i_{h}-1, i_{h}+\right.$ $1, \ldots, d)^{\mathrm{O}}$. By separating from each other the polygons of colours $1, \ldots, i_{h}-1, i_{h}+$ $1, \ldots, d$ which touch in the exceptional vertex, each $d$-cactus $C_{i}$ in turn can be decomposed into $d$-cacti $C_{i, 1}, \ldots, C_{i, i_{h}-1}, C_{i, i_{h}+1}, \ldots, C_{i, d}$ with $C_{i, j} \in \mathcal{C}_{j}$ for all $k$, where $\mathcal{C}_{j}$ denotes the set of all $d$-cacti in which all but one vertex have rotator $(1,2, \ldots, d)^{\mathrm{O}}$, the distinguished vertex being incident to just one face, which is of colour $j$.

Let $\omega_{j}(\mathbf{z})$ denote the generating function for the $d$-cacti in $\mathcal{C}_{j}$, that is,

$$
\omega_{j}(\mathbf{z})=\sum_{C \in \mathcal{C}_{j}} w(C) .
$$

Furthermore, for $i=1,2, \ldots, d$, define the formal power series $P_{i}(u)$ in one variable $u$ via

$$
P_{i}(u)=\sum_{k=1}^{\infty} p_{i, k} u^{k-1} .
$$

Then, by the decomposition (6.13) and the further decomposition of the $K_{h}$ 's that we just described, the contribution of the above $d$-atolls to the generating function 
(6.10) is

$$
\begin{aligned}
& \left(\prod_{j=1}^{\ell} \frac{z_{i_{j} \omega_{i_{j}}(\mathbf{z})}}{\omega_{1}(\mathbf{z}) \cdots \omega_{d}(\mathbf{z})}\left(P_{i_{j}}\left(\frac{\omega_{1}(\mathbf{z}) \cdots \omega_{d}(\mathbf{z})}{\omega_{i_{j}}(\mathbf{z})}\right)-p_{i_{j}, 1}\right)\right) \\
& \quad \times\left(\frac{\prod_{j=1}^{d} z_{j} p_{j, 1}}{\prod_{j=1}^{\ell} z_{i_{j}} p_{i_{j}, 1}}\right) \frac{\left(\omega_{1}(\mathbf{z}) \cdots \omega_{d}(\mathbf{z})\right)^{\ell-1}}{\prod_{j=1}^{\ell} \omega_{i_{j}}(\mathbf{z})} \\
& =\left(\prod_{j=1}^{d} \frac{z_{j} p_{j, 1}}{\omega_{j}(\mathbf{z})}\right) \prod_{j=1}^{\ell}\left(\frac{P_{i_{j}}\left(\frac{\omega_{1}(\mathbf{z}) \cdots \omega_{d}(\mathbf{z})}{\omega_{i_{j}}(\mathbf{z})}\right)}{p_{i_{j}, 1}}-1\right),
\end{aligned}
$$

with the term in the first line corresponding to the contribution of the $K_{j}$ 's, the first term in the second line corresponding to the contribution of the $L_{k}^{(j)}$ 's, and the second term in the second line corresponding to the contribution of the $C_{k}^{(j)}$ 's. These expressions must be summed over $\ell=2,3, \ldots, d$ and all possible choices of $1 \leq i_{1}<i_{2}<\cdots<i_{\ell} \leq d$ to obtain the desired generating function $G(\mathbf{z})$, that is,

$$
\begin{aligned}
G(\mathbf{z}) & =\left(\prod_{j=1}^{d} \frac{z_{j} p_{j, 1}}{\omega_{j}(\mathbf{z})}\right) \sum_{\ell=2}^{d} \sum_{1 \leq i_{1}<i_{2}<\cdots<i_{\ell} \leq d} \prod_{j=1}^{\ell}\left(\frac{P_{i_{j}}\left(\frac{\omega_{1}(\mathbf{z}) \cdots \omega_{d}(\mathbf{z})}{\omega_{i_{j}}(\mathbf{z})}\right)}{p_{i_{j}, 1}}-1\right) \\
& =\left(\prod_{j=1}^{d} \frac{z_{j} p_{j, 1}}{\omega_{j}(\mathbf{z})}\right)\left(\prod_{j=1}^{d} \frac{P_{j}\left(\frac{\omega_{1}(\mathbf{z}) \cdots \omega_{d}(\mathbf{z})}{\omega_{j}(\mathbf{z})}\right)}{p_{j, 1}}-\sum_{j=1}^{d}\left(\frac{P_{j}\left(\frac{\omega_{1}(\mathbf{z}) \cdots \omega_{d}(\mathbf{z})}{\omega_{j}(\mathbf{z})}\right)}{p_{j, 1}}-1\right)-1\right) .
\end{aligned}
$$

Here we have used the elementary identity

$$
\sum_{\ell=0}^{d} \sum_{1 \leq i_{1}<i_{2}<\cdots<i_{\ell} \leq d} X_{i_{1}} X_{i_{2}} \cdots X_{i_{\ell}}=\left(1+X_{1}\right)\left(1+X_{2}\right) \cdots\left(1+X_{d}\right) .
$$

Before we are able to proceed, we must find functional equations for the generating functions $\omega_{j}(\mathbf{z}), j=1,2, \ldots, d$. Given a $d$-cactus $C$ in $\mathcal{C}_{j}$ such that the distinguished vertex is incident to a $k$-gon (of colour $j$ ), we decompose it in a manner analogous to the decomposition of $K_{h}$ above. To be more precise, let $C_{1}, C_{2}, \ldots, C_{k-1}$ be the $d$-cacti incident to this $k$-gon, read in clockwise order, starting with the $d$-cactus to the left of the distinguished vertex. After removal of the $k$-gon, we are left with the ordered collection $C_{1}, C_{2}, \ldots, C_{k-1}$ of $d$-cacti, each having the property that the rotator of all but one vertex is $(1,2, \ldots, d)^{\mathrm{O}}$, the exceptional vertex having rotator $(1, \ldots, j-1, j+1, \ldots, d)^{\mathrm{O}}$. By separating from each other the polygons of colours $1, \ldots, j-1, j+1, \ldots, d$ which touch in the exceptional vertex, each $d$-cactus $C_{i}$ in turn can be decomposed into $d$-cacti $C_{i, 1}, \ldots, C_{i, j-1}, C_{i, j+1}, \ldots, C_{i, d}$ with $C_{i, k} \in \mathcal{C}_{k}$ for all $k$. The upshot of these combinatorial considerations is that

$$
\omega_{j}(\mathbf{z})=z_{j} P_{j}\left(\omega_{1}(\mathbf{z}) \cdots \omega_{d}(\mathbf{z}) / \omega_{j}(\mathbf{z})\right), \quad j=1,2, \ldots, d,
$$

or, equivalently,

$$
z_{j}=\frac{\omega_{j}(\mathbf{z})}{P_{j}\left(\omega_{1}(\mathbf{z}) \cdots \omega_{d}(\mathbf{z}) / \omega_{j}(\mathbf{z})\right)}, \quad j=1,2, \ldots, d .
$$


Using this relation, the expression (6.15) for $G(\mathbf{z})$ may now be further simplified, and we obtain

$G(\mathbf{z})=1-\prod_{j=1}^{d} \frac{p_{j, 1}}{P_{j}\left(\frac{\omega_{1}(\mathbf{z}) \cdots \omega_{d}(\mathbf{z})}{\omega_{j}(\mathbf{z})}\right)} \sum_{j=1}^{d} \frac{P_{j}\left(\frac{\omega_{1}(\mathbf{z}) \cdots \omega_{d}(\mathbf{z})}{\omega_{j}(\mathbf{z})}\right)}{p_{j, 1}}+(d-1) \prod_{j=1}^{d} \frac{p_{j, 1}}{P_{j}\left(\frac{\omega_{1}(\mathbf{z}) \cdots \omega_{d}(\mathbf{z})}{\omega_{j}(\mathbf{z})}\right)}$.

This is substituted in 6.11 to obtain

$$
\begin{aligned}
=-2(n-1)\left\langle\mathbf{z}^{\mathbf{c}} \prod_{i=1}^{d} \prod_{k=0}^{n} p_{i, k+1}^{m_{k}^{(i)}}\right\rangle\left(\prod_{j=1}^{d} \frac{p_{j, 1}}{P_{j}\left(\frac{\omega_{1}(\mathbf{z}) \cdots \omega_{d}(\mathbf{z})}{\omega_{j}(\mathbf{z})}\right)}\right) \sum_{j=1}^{d} \frac{P_{j}\left(\frac{\omega_{1}(\mathbf{z}) \cdots \omega_{d}(\mathbf{z})}{\omega_{j}(\mathbf{z})}\right)}{p_{j, 1}} \\
+2(n-1)(d-1)\left\langle\mathbf{z}^{\mathbf{c}} \prod_{i=1}^{d} \prod_{k=0}^{n} p_{i, k+1}^{m_{k}^{(i)}}\right\rangle \prod_{j=1}^{d} \frac{p_{j, 1}}{P_{j}\left(\frac{\omega_{1}(\mathbf{z}) \cdots \omega_{d}(\mathbf{z})}{\omega_{j}(\mathbf{z})}\right)} .
\end{aligned}
$$

Now the problem is set up for application of the Lagrange-Good inversion formula. Let $f_{i}(\mathbf{z})=z_{i} / P_{i}\left(z_{1} \cdots z_{d} / z_{i}\right), i=1,2, \ldots, d$. If we substitute $f_{i}(\mathbf{z})$ in place of $z_{i}$, $i=1,2, \ldots, d$, in (6.16), and apply Theorem 1 with

$$
g(\mathbf{z})=\left(\prod_{j=1}^{d} \frac{p_{j, 1}}{P_{j}\left(\frac{z_{1} \cdots z_{d}}{z_{j}}\right)}\right) \sum_{j=1}^{d} \frac{P_{j}\left(\frac{z_{1} \cdots z_{d}}{z_{j}}\right)}{p_{j, 1}}
$$

respectively

$$
g(\mathbf{z})=\prod_{j=1}^{d} \frac{p_{j, 1}}{P_{j}\left(\frac{z_{1} \cdots z_{d}}{z_{j}}\right)}
$$

we obtain that

$$
\begin{aligned}
& N_{D_{n}}\left(T_{1}, T_{2}, \ldots, T_{d}\right) \\
&=-2(n-1)\left\langle\mathbf{z}^{\mathbf{0}} \prod_{i=1}^{d} \prod_{k=0}^{n} p_{i, k+1}^{m_{k}^{(i)}}\right\rangle\left(\prod_{i=1}^{d} p_{i, 1}\right)\left(\sum_{j=1}^{d} \frac{z_{j}}{f_{j}(\mathbf{z}) p_{j, 1}}\right) \mathbf{f}^{-\mathbf{c}}(\mathbf{z}) \operatorname{det}_{1 \leq i, k \leq d}\left(\frac{\partial f_{i}}{\partial z_{k}}(\mathbf{z})\right) \\
&+2(n-1)(d-1)\left\langle\mathbf{z}^{\mathbf{0}} \prod_{i=1}^{d} \prod_{k=0}^{n} p_{i, k+1}^{m_{k}^{(i)}}\right\rangle\left(\prod_{i=1}^{d} p_{i, 1}\right) \mathbf{f}^{-\mathbf{c}}(\mathbf{z}) \operatorname{det}_{1 \leq i, k \leq d}\left(\frac{\partial f_{i}}{\partial z_{k}}(\mathbf{z})\right)
\end{aligned}
$$


where $\mathbf{0}$ stands for the vector $(0,0, \ldots, 0)$. We treat the two terms on the right-hand side of (6.17) separately. We begin with the second term:

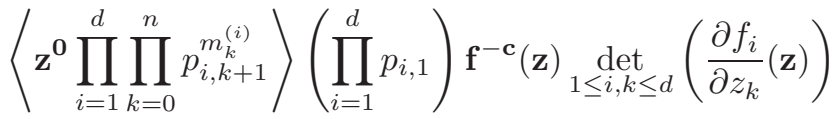

$$
\begin{aligned}
& =\left\langle\mathbf{z}^{\mathbf{c}} \prod_{i=1}^{d} \prod_{k=0}^{n} p_{i, k+1}^{m_{k}^{(i)}}\right\rangle\left(\prod_{i=1}^{d} p_{i, 1}\right) \\
& \times \operatorname{det}_{1 \leq i, k \leq d}\left(\left\{\begin{array}{ll}
P_{i}^{c_{i}-1}\left(\frac{z_{1} \cdots z_{d}}{z_{i}}\right), & i=k \\
-P_{i}^{c_{i}-2}\left(\frac{z_{1} \cdots z_{d}}{z_{i}}\right) & \\
\times P_{i}^{\prime}\left(\frac{z_{1} \cdots z_{d}}{z_{i}}\right) & \frac{z_{1} \cdots z_{d}}{z_{k}}, i \neq k
\end{array}\right\}\right) \\
& =\left\langle\mathbf{z}^{\mathbf{c}} \prod_{i=1}^{d} \prod_{k=0}^{n} p_{i, k+1}^{m_{k}^{(i)}}\right\rangle\left(\prod_{i=1}^{d} p_{i, 1}\right) \\
& \times \operatorname{det}_{1 \leq i, k \leq d}\left(\left\{\begin{array}{ll}
P_{i}^{c_{i}-1}\left(\frac{z_{1} \cdots z_{d}}{z_{i}}\right), & i=k \\
-\left.\frac{1}{c_{i}-1}\left(u \frac{d}{d u} P_{i}^{c_{i}-1}(u)\right)\right|_{u=z_{1} \cdots z_{d} / z_{i}}, & i \neq k
\end{array}\right\}\right) .
\end{aligned}
$$

Reading coefficients, we obtain

$$
\begin{aligned}
\prod_{i=1}^{d}\left(\begin{array}{cc}
c_{i}-1 \\
m_{1}^{(i)}, m_{2}^{(i)}, \ldots, & \left.m_{n}^{(i)}\right)
\end{array}\right) \operatorname{det}_{1 \leq i, k \leq d}\left(\left\{\begin{array}{cc}
1, & i=k \\
-\frac{\mathrm{rk} T_{i}}{c_{i}-1}, & i \neq k
\end{array}\right\}\right) \\
=\prod_{i=1}^{d}\left(\begin{array}{c}
c_{i}-1 \\
\left.m_{1}^{(i)}, m_{2}^{(i)}, \ldots, m_{n}^{(i)}\right)
\end{array}\right) \operatorname{det}_{1 \leq i, k \leq d}\left(1-\chi(i \neq k) \frac{n-1}{c_{i}-1}\right),
\end{aligned}
$$

the second line being due to (6.12). Now we can apply Lemma 2 with $X_{i}=c_{i}-1$ and $Y_{i}=n-1, i=1,2, \ldots, d$. The term

$$
\begin{aligned}
\sum_{i=1}^{d} X_{i}-\sum_{i=2}^{d} Y_{i} & =\sum_{i=1}^{d}\left(c_{i}-1\right)-(d-1)(n-1) \\
& =\sum_{i=1}^{d}\left(n-\operatorname{rk} T_{i}-1\right)-(d-1)(n-1)
\end{aligned}
$$

on the right-hand side of (3.2) simplifies to -1 due to our assumption concerning the sum of the ranks of the types $T_{i}$. Hence, if we use the relation (6.12) once more, the second term on the right-hand side of (6.17) is seen to equal

$$
-2(d-1)(n-1)^{d} \prod_{i=1}^{d} \frac{1}{n-\operatorname{rk} T_{i}-1}\left(\begin{array}{c}
n-\operatorname{rk} T_{i}-1 \\
m_{1}^{(i)}, m_{2}^{(i)}, \ldots, m_{n}^{(i)}
\end{array}\right) .
$$

This explains the third term in the factor in large parentheses in 6.2) and the last term in the factor in large parentheses on the right-hand side of (6.3). 
Finally, we come to the first term on the right-hand side of (6.17). We have

$$
\begin{aligned}
& \left\langle\mathbf{z}^{\mathbf{0}} \prod_{i=1}^{d} \prod_{k=0}^{n} p_{i, k+1}^{m_{k}^{(i)}}\right\rangle\left(\prod_{i=1}^{d} p_{i, 1}\right) \frac{z_{j}}{f_{j}(\mathbf{z}) p_{j, 1}} \mathbf{f}^{-\mathbf{c}}(\mathbf{z}) \operatorname{det}_{1 \leq i, k \leq d}\left(\frac{\partial f_{i}}{\partial z_{k}}(\mathbf{z})\right) \\
& =\left\langle\mathbf{z}^{\mathbf{c}} \prod_{i=1}^{d} \prod_{k=0}^{n} p_{i, k+1}^{m_{k}^{(i)}}\right\rangle\left(\prod_{\substack{i=1 \\
i \neq j}}^{d} p_{i, 1}\right) \operatorname{det}_{1 \leq i, k \leq d}\left(\left\{\begin{array}{cc}
P_{i}^{c_{i}-1+\chi(i=j)}\left(\frac{z_{1} \cdots z_{d}}{z_{i}}\right), & i=k \\
-P_{i}^{c_{i}-2+\chi(i=j)}\left(\frac{z_{1} \cdots z_{d}}{z_{i}}\right) & \\
\times P_{i}^{\prime}\left(\frac{z_{1} \cdots z_{d}}{z_{i}}\right) \frac{z_{1} \cdots z_{d}}{z_{k}}, & i \neq k
\end{array}\right\}\right) \\
& =\left\langle\mathbf{z}^{\mathbf{c}} \prod_{i=1}^{d} \prod_{k=0}^{n} p_{i, k+1}^{m_{k}^{(i)}}\right\rangle\left(\prod_{\substack{i=1 \\
i \neq j}}^{d} p_{i, 1}\right) \\
& \times \operatorname{det}_{1 \leq i, k \leq d}\left(\left\{\begin{array}{ll}
P_{i}^{c_{i}-1+\chi(i=j)}\left(\frac{z_{1} \cdots z_{d}}{z_{i}}\right), & i=k \\
-\left.\frac{1}{c_{i}-1+\chi(i=j)}\left(u \frac{d}{d u} P_{i}^{c_{i}-1+\chi(i=j)}(u)\right)\right|_{u=z_{1} \cdots z_{d} / z_{i}}, & i \neq k
\end{array}\right\}\right) .
\end{aligned}
$$

Reading coefficients, we obtain

$$
\begin{gathered}
\prod_{i=1}^{d}\left(\begin{array}{c}
c_{i}-1+\chi(i=j) \\
m_{1}^{(i)}, m_{2}^{(i)}, \ldots, m_{n}^{(i)}
\end{array}\right) \operatorname{det}_{1 \leq i, k \leq d}\left(\left\{\begin{array}{cc}
1, & i=k \\
-\frac{\operatorname{rk} T_{i}}{c_{i}-1+\chi(i=j)}, & i \neq k
\end{array}\right\}\right) \\
=\prod_{i=1}^{d}\left(\begin{array}{c}
c_{i}-1+\chi(i=j) \\
m_{1}^{(i)}, m_{2}^{(i)}, \ldots, m_{n}^{(i)}
\end{array}\right) \operatorname{det}_{1 \leq i, k \leq d}\left(\left\{\begin{array}{cc}
1-\chi(j \neq k) \frac{n}{c_{j}}, & i=j \\
1-\chi(i \neq k) \frac{n-1}{c_{i}-1}, & i \neq j
\end{array}\right\}\right),
\end{gathered}
$$

the second line being due to (6.12). Now we can apply Corollary 3 with $r=j$, $X_{i}=c_{i}-1, i=1, \ldots, j-1, j+1, \ldots, d, X_{j}=c_{j}, Y=n-1$, and $Z=n$. The term

$$
\begin{aligned}
Z \sum_{i=1}^{d} & X_{i}+(Y-Z) X_{j}-(d-1) Y Z \\
& =n\left(1+\sum_{i=1}^{d}\left(c_{i}-1\right)\right)-c_{j}-(d-1)(n-1) n \\
& =n \sum_{i=1}^{d}\left(n-\operatorname{rk} T_{i}-1\right)+n-c_{j}-(d-1)(n-1) n
\end{aligned}
$$

on the right-hand side of (3.2) simplifies to $-c_{j}$ due to our assumption concerning the sum of the ranks of types $T_{i}$. Hence, if we use the relation (6.12) once more, the second term on the right-hand side of (6.17) is seen to equal the sum over $j=1,2, \ldots, d$ of

$$
2(n-1)^{d-1}\left(\begin{array}{c}
n-\operatorname{rk} T_{j} \\
m_{1}^{(j)}, m_{2}^{(j)}, \ldots, m_{n}^{(j)}
\end{array}\right) \prod_{\substack{i=1 \\
i \neq j}}^{d} \frac{1}{n-\operatorname{rk} T_{i}-1}\left(\begin{array}{c}
n-\operatorname{rk} T_{i}-1 \\
m_{1}^{(i)}, m_{2}^{(i)}, \ldots, m_{n}^{(i)}
\end{array}\right) .
$$


This explains the first terms in the factors in large parentheses on the right-hand sides of (6.2) and (6.3).

The proof of the theorem is complete.

Combining the previous theorem with the summation formula of Lemma 4 we can now derive compact formulae for all type $D_{n}$ decomposition numbers.

Theorem 10. (i) Let types $T_{1}, T_{2}, \ldots, T_{d}$ be given, where

$$
T_{i}=A_{1}^{m_{1}^{(i)}} * A_{2}^{m_{2}^{(i)}} * \cdots * A_{n}^{m_{n}^{(i)}}, \quad i=1,2, \ldots, j-1, j+1, \ldots, d,
$$

and

$$
T_{j}=D_{\alpha} * A_{1}^{m_{1}^{(j)}} * A_{2}^{m_{2}^{(j)}} * \cdots * A_{n}^{m_{n}^{(j)}},
$$

for some $\alpha \geq 2$. Then

$$
\begin{aligned}
& N_{D_{n}}^{c o m b}\left(T_{1}, T_{2}, \ldots, T_{d}\right)=(n-1)^{d-1}\left(\begin{array}{c}
n-1 \\
\operatorname{rk} T_{1}+\operatorname{rk} T_{2}+\cdots+\operatorname{rk} T_{d}-1
\end{array}\right) \\
& \quad \times\left(\begin{array}{c}
n-\operatorname{rk} T_{j} \\
m_{1}^{(j)}, m_{2}^{(j)}, \ldots, m_{n}^{(j)}
\end{array}\right) \prod_{\substack{i=1 \\
i \neq j}}^{d} \frac{1}{n-\operatorname{rk} T_{i}-1}\left(\begin{array}{c}
n-\operatorname{rk} T_{i}-1 \\
m_{1}^{(i)}, m_{2}^{(i)}, \ldots, m_{n}^{(i)}
\end{array}\right),
\end{aligned}
$$

where the multinomial coefficient is defined as in Lemma 4. For $\alpha \geq 4$, the number $N_{D_{n}}\left(T_{1}, T_{2}, \ldots, T_{d}\right)$ is given by the same formula.

(ii) Let types $T_{1}, T_{2}, \ldots, T_{d}$ be given, where

$$
T_{i}=A_{1}^{m_{1}^{(i)}} * A_{2}^{m_{2}^{(i)}} * \cdots * A_{n}^{m_{n}^{(i)}}, \quad i=1,2, \ldots, d .
$$

Then

$$
\begin{aligned}
& N_{D_{n}}^{c o m b}\left(T_{1}, T_{2}, \ldots, T_{d}\right)=(n-1)^{d-1}\left(\begin{array}{c}
n-1 \\
\operatorname{rk} T_{1}+\operatorname{rk} T_{2}+\cdots+\operatorname{rk} T_{d}-1
\end{array}\right) \\
& \times\left(2 \sum_{j=1}^{d}\left(\begin{array}{c}
n-\mathrm{rk} T_{j} \\
m_{1}^{(j)}, m_{2}^{(j)}, \ldots, m_{n}^{(j)}
\end{array}\right)\left(\prod_{\substack{i=1 \\
i \neq j}}^{d} \frac{1}{n-\operatorname{rk} T_{i}-1}\left(\begin{array}{c}
n-\operatorname{rk} T_{i}-1 \\
m_{1}^{(i)}, m_{2}^{(i)}, \ldots, m_{n}^{(i)}
\end{array}\right)\right)\right. \\
& +\left(\frac{\left(n-\sum_{\ell=1}^{d} \mathrm{rk} T_{\ell}\right)\left(n-1-\sum_{\ell=1}^{d} \mathrm{rk} T_{\ell}\right)}{\sum_{\ell=1}^{d} \mathrm{rk} T_{\ell}}-2(d-2)(n-1)\right) \\
& \left.\prod_{i=1}^{d} \frac{1}{n-\operatorname{rk} T_{i}-1}\left(\begin{array}{c}
n-\operatorname{rk} T_{i}-1 \\
m_{1}^{(i)}, m_{2}^{(i)}, \ldots, m_{n}^{(i)}
\end{array}\right)\right),
\end{aligned}
$$


whereas

$$
\begin{aligned}
& N_{D_{n}}\left(T_{1}, T_{2}, \ldots, T_{d}\right)=(n-1)^{d-1}\left(\begin{array}{c}
n-1 \\
\operatorname{rk} T_{1}+\operatorname{rk} T_{2}+\cdots+\operatorname{rk} T_{d}-1
\end{array}\right) \\
& \times\left(\sum _ { j = 1 } ^ { d } ( \prod _ { \substack { i = 1 \\
i \neq j } } ^ { d } \frac { 1 } { n - \operatorname { r k } T _ { i } - 1 } ( \begin{array} { c } 
{ n - \operatorname { r k } T _ { i } - 1 } \\
{ m _ { 1 } ^ { ( i ) } , m _ { 2 } ^ { ( i ) } , \ldots , m _ { n } ^ { ( i ) } }
\end{array} ) ) \left(2\left(\begin{array}{c}
n-\operatorname{rk} T_{j} \\
m_{1}^{(j)}, m_{2}^{(j)}, \ldots, m_{n}^{(j)}
\end{array}\right)\right.\right. \\
& \left.+\left(\begin{array}{c}
n-\operatorname{rk} T_{j} \\
m_{1}^{(j)}, m_{2}^{(j)}, m_{3}^{(j)}-1, m_{4}^{(j)}, \ldots, m_{n}^{(j)}
\end{array}\right)+\left(\begin{array}{c}
n-\operatorname{rk} T_{j} \\
m_{1}^{(j)}-2, m_{2}^{(j)}, \ldots, m_{n}^{(j)}
\end{array}\right)\right) \\
& +\left(\frac{\left(n-\sum_{\ell=1}^{d} \mathrm{rk} T_{\ell}\right)\left(n-1-\sum_{\ell=1}^{d} \mathrm{rk} T_{\ell}\right)}{\sum_{\ell=1}^{d} \mathrm{rk} T_{\ell}}-2(d-2)(n-1)\right) \\
& \left.\cdot \prod_{i=1}^{d} \frac{1}{n-\operatorname{rk} T_{i}-1}\left(\begin{array}{c}
n-\operatorname{rk} T_{i}-1 \\
m_{1}^{(i)}, m_{2}^{(i)}, \ldots, m_{n}^{(i)}
\end{array}\right)\right) .
\end{aligned}
$$

(iii) All of the other decomposition numbers $N_{D_{n}}\left(T_{1}, T_{2}, \ldots, T_{d}\right)$ and $N_{D_{n}}^{\text {comb }}\left(T_{1}, T_{2}, \ldots, T_{d}\right)$ are zero.

Remark. The caveats on interpretations of the formulae in Theorem 9 for critical choices of the parameters (cf. the Remark after the statement of that theorem) also apply to the formulae of Theorem 10

Proof. We proceed in a manner similar to the proof of Theorem 8 . If we write $r$ for $n-\operatorname{rk} T_{1}-\operatorname{rk} T_{2}-\cdots-\operatorname{rk} T_{d}$ and set $\Phi=D_{n}$, then relation (2.3) becomes

$$
N_{D_{n}}\left(T_{1}, T_{2}, \ldots, T_{d}\right)=\sum_{T: \mathrm{rk} T=r} N_{D_{n}}\left(T_{1}, T_{2}, \ldots, T_{d}, T\right)
$$

with the same relation holding for $N_{D_{n}}^{\text {comb }}$ in place of $N_{D_{n}}$.

In order to prove (6.18), we let $T=A_{1}^{m_{1}} * A_{2}^{m_{2}} * \cdots * A_{n}^{m_{n}}$ and use (6.1) in (6.21) to obtain

$$
\begin{array}{r}
N_{D_{n}}^{\mathrm{comb}}\left(T_{1}, T_{2}, \ldots, T_{d}\right)=\sum_{m_{1}+2 m_{2}+\cdots+n m_{n}=r}(n-1)^{d} \frac{1}{n-r-1}\left(\begin{array}{c}
n-r-1 \\
m_{1}, m_{2}, \ldots, m_{n}
\end{array}\right) \\
\cdot\left(\begin{array}{c}
n-\mathrm{rk} T_{j} \\
m_{1}^{(j)}, m_{2}^{(j)}, \ldots, m_{n}^{(j)}
\end{array}\right) \prod_{\substack{i=1 \\
i \neq j}}^{d} \frac{1}{n-\mathrm{rk} T_{i}-1}\left(\begin{array}{c}
n-\operatorname{rk} T_{i}-1 \\
m_{1}^{(i)}, m_{2}^{(i)}, \ldots, m_{n}^{(i)}
\end{array}\right) .
\end{array}
$$

If we use (3.4) with $M=n-r-1$, we arrive at our claim after little simplification.

Next we prove (6.19). In contrast to the previous argument, here the summation on the right-hand side of (6.21) must be taken over all types $T$ of the form $T=$ $D_{\alpha} * A_{1}^{m_{1}} * A_{2}^{m_{2}} * \cdots * A_{n}^{m_{n}}, \alpha \geq 2$, as well as of the form $T=A_{1}^{m_{1}} * A_{2}^{m_{2}} * \cdots * A_{n}^{m_{n}}$. 
For the sum over the former types, we have to substitute (6.1) in (6.21) to get

$$
\begin{aligned}
& \sum_{\alpha=2}^{n} \sum_{m_{1}+2 m_{2}+\cdots+n m_{n}=r-\alpha}(n-1)^{d}\left(\begin{array}{c}
n-r \\
m_{1}, m_{2}, \ldots, m_{n}
\end{array}\right) \\
& \cdot \prod_{i=1}^{d} \frac{1}{n-\operatorname{rk} T_{i}-1}\left(\begin{array}{c}
n-\operatorname{rk} T_{i}-1 \\
m_{1}^{(i)}, m_{2}^{(i)}, \ldots, m_{n}^{(i)}
\end{array}\right) .
\end{aligned}
$$

On the other hand, for the sum over the latter types, we have to substitute (6.2) in (6.21) to get

$$
\begin{aligned}
& 2 \sum_{m_{1}+2 m_{2}+\cdots+n m_{n}=r}(n-1)^{d}\left(\begin{array}{c}
n-r \\
m_{1}, m_{2}, \ldots, m_{n}
\end{array}\right) \\
& \cdot \prod_{i=1}^{d} \frac{1}{n-\operatorname{rk} T_{i}-1}\left(\begin{array}{c}
n-\operatorname{rk} T_{i}-1 \\
m_{1}^{(i)}, m_{2}^{(i)}, \ldots, m_{n}^{(i)}
\end{array}\right) \\
& +\sum_{m_{1}+2 m_{2}+\cdots+n m_{n}=r}(n-1)^{d} \frac{1}{n-r-1}\left(\begin{array}{c}
n-r-1 \\
m_{1}, m_{2}, \ldots, m_{n}
\end{array}\right) \\
& \cdot\left(2 \sum_{j=1}^{d}\left(\begin{array}{c}
n-\mathrm{rk} T_{j} \\
m_{1}^{(j)}, m_{2}^{(j)}, \ldots, m_{n}^{(j)}
\end{array}\right) \prod_{\substack{i=1 \\
i \neq j}}^{d} \frac{1}{n-\mathrm{rk} T_{i}-1}\left(\begin{array}{c}
n-\mathrm{rk} T_{i}-1 \\
m_{1}^{(i)}, m_{2}^{(i)}, \ldots, m_{n}^{(i)}
\end{array}\right)\right. \\
& \left.-2(d-1)(n-1) \prod_{i=1}^{d} \frac{1}{n-\operatorname{rk} T_{i}-1}\left(\begin{array}{c}
n-\mathrm{rk} T_{i}-1 \\
m_{1}^{(i)}, m_{2}^{(i)}, \ldots, m_{n}^{(i)}
\end{array}\right)\right) .
\end{aligned}
$$

We simplify (6.22) by using (3.4) with $r$ replaced by $r-\alpha$ and $M=n-r$ and by subsequently applying the elementary summation formula

$$
\sum_{\alpha=2}^{n}\left(\begin{array}{c}
n-\alpha-1 \\
r-\alpha
\end{array}\right)=\sum_{\alpha=2}^{n}\left(\begin{array}{c}
n-\alpha-1 \\
n-r-1
\end{array}\right)=\left(\begin{array}{l}
n-2 \\
n-r
\end{array}\right)=\left(\begin{array}{l}
n-2 \\
r-2
\end{array}\right)
$$

The expression which we obtain in this way explains the fraction in the third line of (6.19) multiplied by the expression in the last line. On the other hand, we simplify the sums in (6.23) by using (3.4) with $M=n-r$, respectively $M=n-r-1$. 
Thus, the expression (6.23) becomes

$$
\begin{aligned}
2(n-1)^{d}\left(\begin{array}{c}
n-1 \\
r
\end{array}\right) \prod_{i=1}^{d} \frac{1}{n-\operatorname{rk} T_{i}-1}\left(\begin{array}{c}
n-\operatorname{rk} T_{i}-1 \\
m_{1}^{(i)}, m_{2}^{(i)}, \ldots, m_{n}^{(i)}
\end{array}\right) \\
+(n-1)^{d-1}\left(\begin{array}{c}
n-1 \\
r
\end{array}\right)\left(2 \sum_{j=1}^{d}\left(\begin{array}{c}
n-\operatorname{rk} T_{j} \\
m_{1}^{(j)}, m_{2}^{(j)}, \ldots, m_{n}^{(j)}
\end{array}\right)\right. \\
\cdot \prod_{i=1}^{d} \frac{1}{n-\operatorname{rk} T_{i}-1}\left(\begin{array}{c}
n-\operatorname{rk} T_{i}-1 \\
m_{1}^{(i)}, m_{2}^{(i)}, \ldots, m_{n}^{(i)}
\end{array}\right) \\
\left.-2(d-1)(n-1) \prod_{i=1}^{d} \frac{1}{n-\operatorname{rk} T_{i}-1}\left(\begin{array}{c}
n-\operatorname{rk} T_{i}-1 \\
m_{1}^{(i)}, m_{2}^{(i)}, \ldots, m_{n}^{(i)}
\end{array}\right)\right)
\end{aligned}
$$

which explains the expression in the second line of (6.19) and the second expression in the third line of (6.19) multiplied by the expression in the last line.

The proof of (6.20) is analogous, using (6.3) instead of (6.2). We leave the details to the reader.

\section{Generalised NON-CrOSSING PARTitions}

In this section we recall the definition of Armstrong's [1 generalised non-crossing partitions poset, and its combinatorial realisation from [1] and 29] for the types $A_{n}, B_{n}$, and $D_{n}$.

Let $\Phi$ again be a finite root system of rank $n$, and let $W=W(\Phi)$ be the corresponding reflection group. We first define the non-crossing partition lattice $N C(\Phi)$ (cf. [8, 15]). Let $c$ be a Coxeter element in $W$. Then $N C(\Phi)$ is defined to be the restriction of the partial order $\leq_{T}$ from Section 2 to the set of all elements which are less than or equal to $c$ in this partial order. This definition makes sense since any two Coxeter elements in $W$ are conjugate to each other; the induced inner automorphism then restricts to an isomorphism of the posets corresponding to the two Coxeter elements. It can be shown that $N C(\Phi)$ is in fact a lattice (see [16] for a uniform proof) and moreover self-dual (this is obvious from the definition). Clearly, the minimal element in $N C(\Phi)$ is the identity element in $W$, which we denote by $\varepsilon$, and the maximal element in $N C(\Phi)$ is the chosen Coxeter element $c$. The term "non-crossing partition lattice" is used because $N C\left(A_{n}\right)$ is isomorphic to the lattice of non-crossing partitions of $\{1,2, \ldots, n+1\}$, originally introduced by Kreweras 30. (see also 20] and below), and since also $N C\left(B_{n}\right)$ and $N C\left(D_{n}\right)$ can be realised as lattices of non-crossing partitions (see [5, 32] and below).

In addition to a fixed root system, the definition of Armstrong's generalised noncrossing partitions requires a fixed positive integer $m$. The poset of $m$-divisible noncrossing partitions associated to the root system $\Phi$ has as ground set the following subset of $(N C(\Phi))^{m+1}$ :

$$
\begin{aligned}
N C^{m}(\Phi)=\left\{\left(w_{0} ; w_{1}, \ldots, w_{m}\right)\right. & : w_{0} w_{1} \cdots w_{m}=c \text { and } \\
& \left.\ell_{T}\left(w_{0}\right)+\ell_{T}\left(w_{1}\right)+\cdots+\ell_{T}\left(w_{m}\right)=\ell_{T}(c)\right\} .
\end{aligned}
$$

The order relation is defined by

$$
\left(u_{0} ; u_{1}, \ldots, u_{m}\right) \leq\left(w_{0} ; w_{1}, \ldots, w_{m}\right) \quad \text { if and only if } \quad u_{i} \geq_{T} w_{i}, 1 \leq i \leq m .
$$


(According to this definition, $u_{0}$ and $w_{0}$ need not be related in any way. However, it follows from [1, Lemma 3.4.7] that, in fact, $u_{0} \leq_{T} w_{0}$.) The poset $N C^{m}(\Phi)$ is graded by the rank function

$$
\operatorname{rk}\left(\left(w_{0} ; w_{1}, \ldots, w_{m}\right)\right)=\ell_{T}\left(w_{0}\right) .
$$

Thus, there is a unique maximal element, namely $(c ; \varepsilon, \ldots, \varepsilon)$, where $\varepsilon$ stands for the identity element in $W$, but, for $m>1$, there are many different minimal elements. In particular, $N C^{m}(\Phi)$ has no least element if $m>1$; hence, $N C^{m}(\Phi)$ is not a lattice for $m>1$. (It is, however, a graded join-semilattice; see [1, Theorem 3.4.4].)

In what follows, we shall use the notions "generalised non-crossing partitions" and " $m$-divisible non-crossing partitions" interchangeably, where the latter notion will be employed particularly in contexts in which we want to underline the presence of the parameter $m$.

In the remainder of this section, we explain combinatorial realisations of the $m$-divisible non-crossing partitions of types $A_{n-1}, B_{n}$, and $D_{n}$. In order to be able to do so, we need to recall the definition of Kreweras' non-crossing partitions of $\{1,2, \ldots, N\}$, his "partitions non croisées d'un cycle" of [30]. We place $N$ vertices around a cycle and label them $1,2, \ldots, N$ in clockwise order. The circular representation of a partition of the set $\{1,2, \ldots, N\}$ is the geometric object which arises by representing each block $\left\{i_{1}, i_{2}, \ldots, i_{k}\right\}$ of the partition, where $i_{1}<i_{2}<\cdots<i_{k}$, by the polygon consisting of the vertices labelled $i_{1}, i_{2}, \ldots, i_{k}$ and edges which connect these vertices in clockwise order. A partition of $\{1,2, \ldots, N\}$ is called non-crossing if any two edges in its circular representation are disjoint. Figure 7 shows the non-crossing partition

$$
\{\{1,2,21\},\{3,19,20\},\{4,5,6\},\{7,17,18\},\{8,9,10,11,12,13,14,15,16\}\}
$$

of $\{1,2, \ldots, 21\}$. There is a natural partial order on Kreweras' non-crossing partitions defined by refinement: a partition $\pi_{1}$ is less than or equal to the partition $\pi_{2}$ if every block of $\pi_{1}$ is contained in some block of $\pi_{2}$.

If $\Phi=A_{n-1}$, then the $m$-divisible non-crossing partitions are in bijection with the Kreweras-type non-crossing partitions of the set $\{1,2, \ldots, m n\}$, in which all the block sizes are divisible by $m$. We denote the latter set of non-crossing partitions by $\widetilde{N C^{m}}\left(A_{n-1}\right)$. This was first considered by Edelman in [18. In fact, Figure 7 shows an example of a 3 -divisible non-crossing partition of type $A_{20}$.

Given an element $\left(w_{0} ; w_{1}, \ldots, w_{m}\right) \in N C^{m}\left(A_{n-1}\right)$, the bijection, $\nabla_{A_{n-1}}^{m}$ say, from [1, Theorem 4.3.8] works by "blowing up" $w_{1}, w_{2}, \ldots, w_{m}$, thereby "interleaving" them, and then "gluing" them together by an operation which is called the Kreweras complement in 11. More precisely, for $i=1,2, \ldots, m$, let $\tau_{m, i}$ be the transformation which maps a permutation $w \in S_{n}$ to a permutation $\tau_{m, i}(w) \in$ $S_{m n}$ by letting

$$
\left(\tau_{m, i}(w)\right)(m k+i-m)=m w(k)+i-m, \quad k=1,2, \ldots, n,
$$

and $\left(\tau_{m, i}(w)\right)(l)=l$ for all $l \not \equiv i(\bmod m)$. At this point, the reader should recall from Section 2 that $W\left(A_{n-1}\right)$ is the symmetric group $S_{n}$ and that the standard choice of a Coxeter element in $W\left(A_{n-1}\right)=S_{n}$ is $c=(1,2, \ldots, n)$. With this choice of Coxeter element, the announced bijection maps $\left(w_{0} ; w_{1}, \ldots, w_{m}\right) \in N C^{m}\left(A_{n-1}\right)$ 


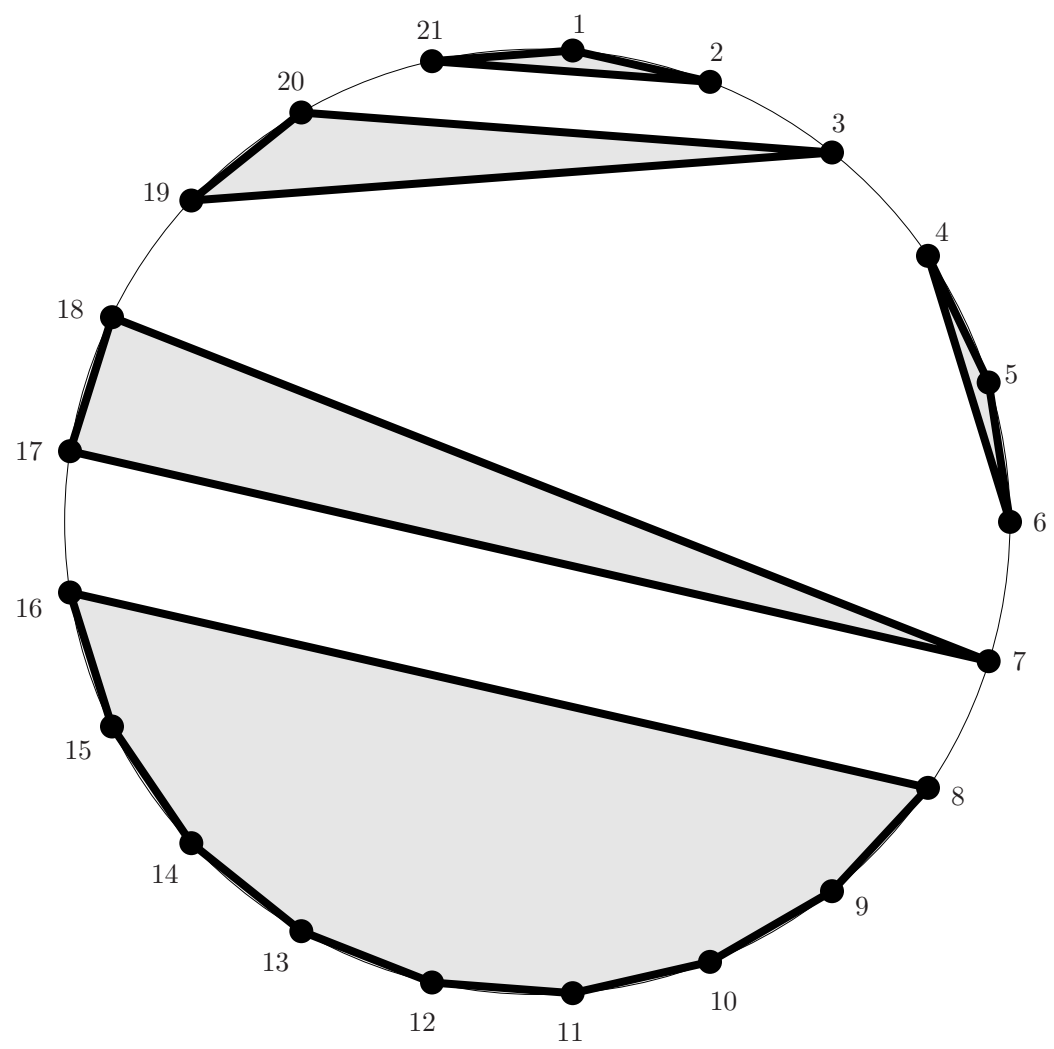

Figure 7. Combinatorial realisation of a 3-divisible non-crossing partition of type $A_{6}$

to

$$
\begin{aligned}
\nabla_{A_{n-1}}^{m}\left(w_{0} ; w_{1}, \ldots, w_{m}\right)= & (1,2, \ldots, m n)\left(\tau_{m, 1}\left(w_{1}\right)\right)^{-1}\left(\tau_{m, 2}\left(w_{2}\right)\right)^{-1} \\
& \cdots\left(\tau_{m, m}\left(w_{m}\right)\right)^{-1} .
\end{aligned}
$$

We refer the reader to [1, Sec. 4.3.2] for the details. For example, let $n=7, m=3$, $w_{0}=(4,5,6), w_{1}=(3,6), w_{2}=(1,7)$, and $w_{3}=(1,2,6)$. Then $\left(w_{0} ; w_{1}, w_{2}, w_{3}\right)$ is mapped to

$$
\begin{aligned}
\nabla_{A_{6}}^{3}\left(w_{0} ; w_{1}, w_{2}, w_{3}\right) & =(1,2, \ldots, 21)(7,16)(2,20)(18,6,3) \\
& =(1,2,21)(3,19,20)(4,5,6)(7,17,18)(8,9, \ldots, 16) .
\end{aligned}
$$

Figure 7 shows the graphical representation of (7.3) on the circle, in which we represent a cycle $\left(i_{1}, i_{2}, \ldots, i_{k}\right)$ as a polygon consisting of the vertices labelled $i_{1}, i_{2}, \ldots, i_{k}$ and edges which connect these vertices in clockwise order.

It is shown in [1, Theorem 4.3.8] that $\nabla_{A_{n-1}}^{m}$ is in fact an isomorphism between the posets $N C^{m}\left(A_{n-1}\right)$ and $\widetilde{N C}^{m}\left(A_{n-1}\right)$. Furthermore, it is proved in [1, Theorem 4.3.13] that

$$
c_{i}\left(w_{0}\right)=b_{i}\left(\nabla_{A_{n-1}}^{m}\left(w_{0} ; w_{1}, \ldots, w_{m}\right)\right), \quad i=1,2, \ldots, n,
$$

where $c_{i}\left(w_{0}\right)$ denotes the number of cycles of length $i$ of $w_{0}$ and $b_{i}(\pi)$ denotes the number of blocks of size $m i$ in the non-crossing partition $\pi$. 


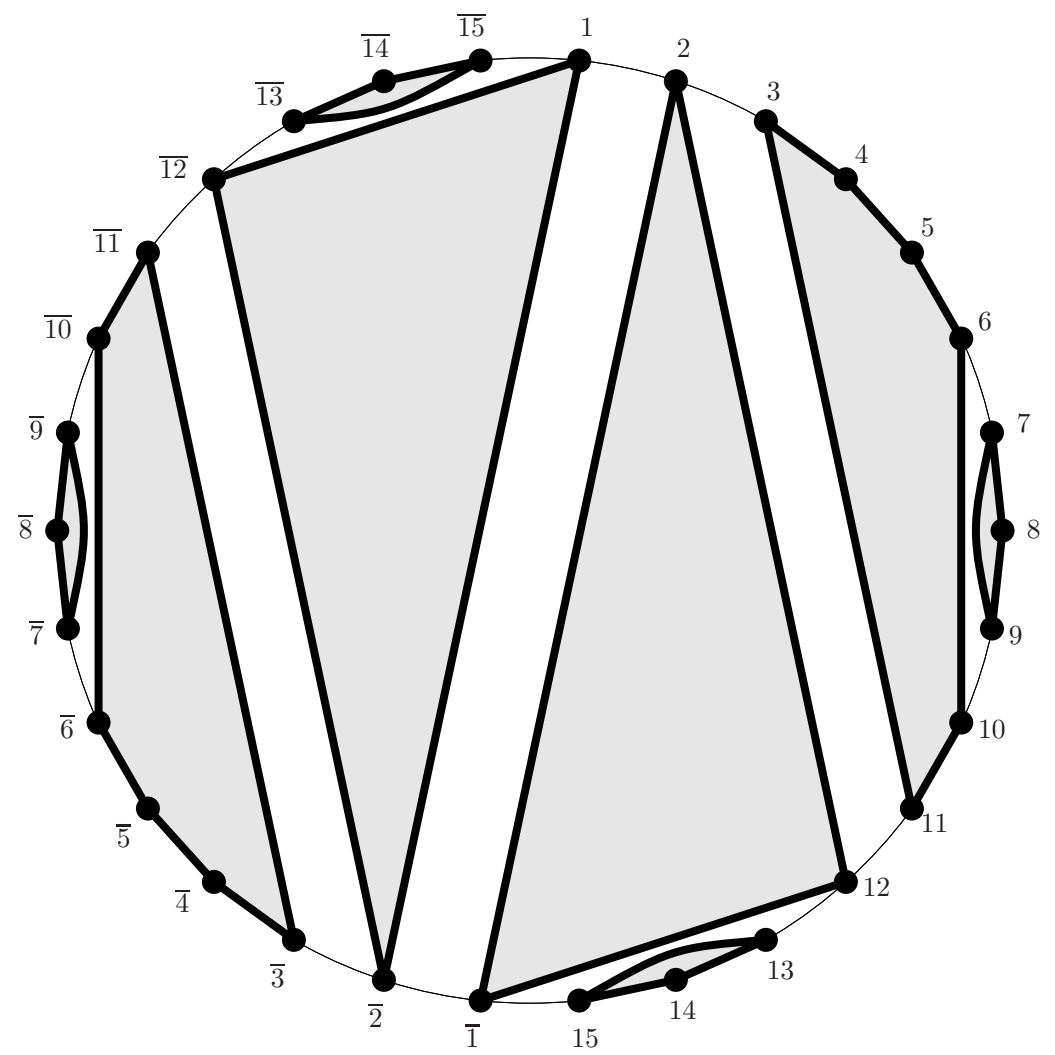

Figure 8. Combinatorial realisation of a 3-divisible non-crossing partition of type $B_{5}$

If $\Phi=B_{n}$, the $m$-divisible non-crossing partitions are in bijection with Krewerastype non-crossing partitions $\pi$ of the set $\{1,2, \ldots, m n, \overline{1}, \overline{2}, \ldots, \overline{m n}\}$, in which all the block sizes are divisible by $m$, and which have the property that if $B$ is a block of $\pi$, then also $\bar{B}:=\{\bar{x}: x \in B\}$ is a block of $\pi$. (Here, as earlier, we adopt the convention that $\overline{\bar{x}}=x$ for all $x$.) We denote the latter set of non-crossing partitions by $\widetilde{N C}^{m}\left(B_{n}\right)$. A block $B$ with $\bar{B}=B$ is called a zero block. A non-crossing partition in $\widetilde{N C}\left(B_{n}\right)$ can only have at most one zero block. Figures 8 and 9 give examples of 3 -divisible non-crossing partitions of type $B_{5}$. Figure 8 shows one without a zero block, while Figure 9 shows one with a zero block. Clearly, the condition that $B$ is a block of the partition if and only if $\bar{B}$ is a block translates into the condition that the geometric realisation of the partition is invariant under rotation by $180^{\circ}$.

Given an element $\left(w_{0} ; w_{1}, \ldots, w_{m}\right) \in N C^{m}\left(B_{n}\right)$, the bijection, $\nabla_{B_{n}}^{m}$ say, from [1. Theorem 4.5.6] works in the same way as for $N C^{m}\left(A_{n-1}\right)$. That is, recalling from Section 2 that $W\left(B_{n}\right)$ can be combinatorially realised as a subgroup of the group of permutations of $\{1,2, \ldots, n, \overline{1}, \overline{2}, \ldots, \bar{n}\}$ and that, in this realisation, the standard choice of a Coxeter element is $c=[1,2, \ldots, n]=(1,2, \ldots, n, \overline{1}, \overline{2}, \ldots, \bar{n})$, the announced bijection maps $\left(w_{0} ; w_{1}, \ldots, w_{m}\right) \in N C^{m}\left(B_{n}\right)$ to

$\nabla_{B_{n}}^{m}\left(w_{0} ; w_{1}, \ldots, w_{m}\right)=[1,2, \ldots, m n]\left(\bar{\tau}_{m, 1}\left(w_{1}\right)\right)^{-1}\left(\bar{\tau}_{m, 2}\left(w_{2}\right)\right)^{-1} \cdots\left(\bar{\tau}_{m, m}\left(w_{m}\right)\right)^{-1}$, 




FiguRE 9. A 3-divisible non-crossing partition of type $B_{5}$ with zero block

where $\bar{\tau}_{m, i}$ is the obvious extension of the above transformations $\tau_{m, i}$. Namely, we let

$$
\left(\bar{\tau}_{m, i}(w)\right)(m k+i-m)=m w(k)+i-m, \quad k=1,2, \ldots, n, \overline{1}, \overline{2}, \ldots, \bar{n},
$$

and $\left(\bar{\tau}_{m, i}(w)\right)(l)=l$ and $\left(\bar{\tau}_{m, i}(w)\right)(\bar{l})=\bar{l}$ for all $l \not \equiv i(\bmod m)$, where $m \bar{k}+i-m$ is identified with $\overline{m k+i-m}$ for all $k$ and $i$. We refer the reader to [1, Sec. 4.5] for the details. For example, let $n=5, m=3, w_{0}=((2,4)), w_{1}=[1]=(1, \overline{1})$, $w_{2}=((1,4))$, and $w_{3}=((2,3))((4,5))$. Then $\left(w_{0} ; w_{1}, w_{2}, w_{3}\right)$ is mapped to

$$
\nabla_{B_{5}}^{3}\left(w_{0} ; w_{1}, w_{2}, w_{3}\right)=[1,2, \ldots, 15][1]((2,11))((6,9))((12,15))
$$

$$
=((1, \overline{2}, \overline{12}))((3,4,5,6,10,11))((7,8,9))((13,14,15)) .
$$

Figure 8 shows the graphical representation of (7.5).

It is shown in [1, Theorem 4.5.6] that $\nabla_{B_{n}}^{m}$ is in fact an isomorphism between the posets $N C^{m}\left(B_{n}\right)$ and $\widetilde{N C}\left(B_{n}\right)$. Furthermore, it is proved in 1, proof of Theorem 4.3.13] that

$$
c_{i}\left(w_{0}\right)=b_{i}\left(\nabla_{B_{n}}^{m}\left(w_{0} ; w_{1}, \ldots, w_{m}\right)\right), \quad i=1,2, \ldots, n,
$$

where $c_{i}\left(w_{0}\right)$ denotes the number of type $A$ cycles (recall the corresponding terminology from Section 4 ) of length $i$ of $w_{0}$ and $b_{i}(\pi)$ denotes one half of the number of non-zero blocks of size $m i$ in the non-crossing partition $\pi$. (Recall that non-zero 


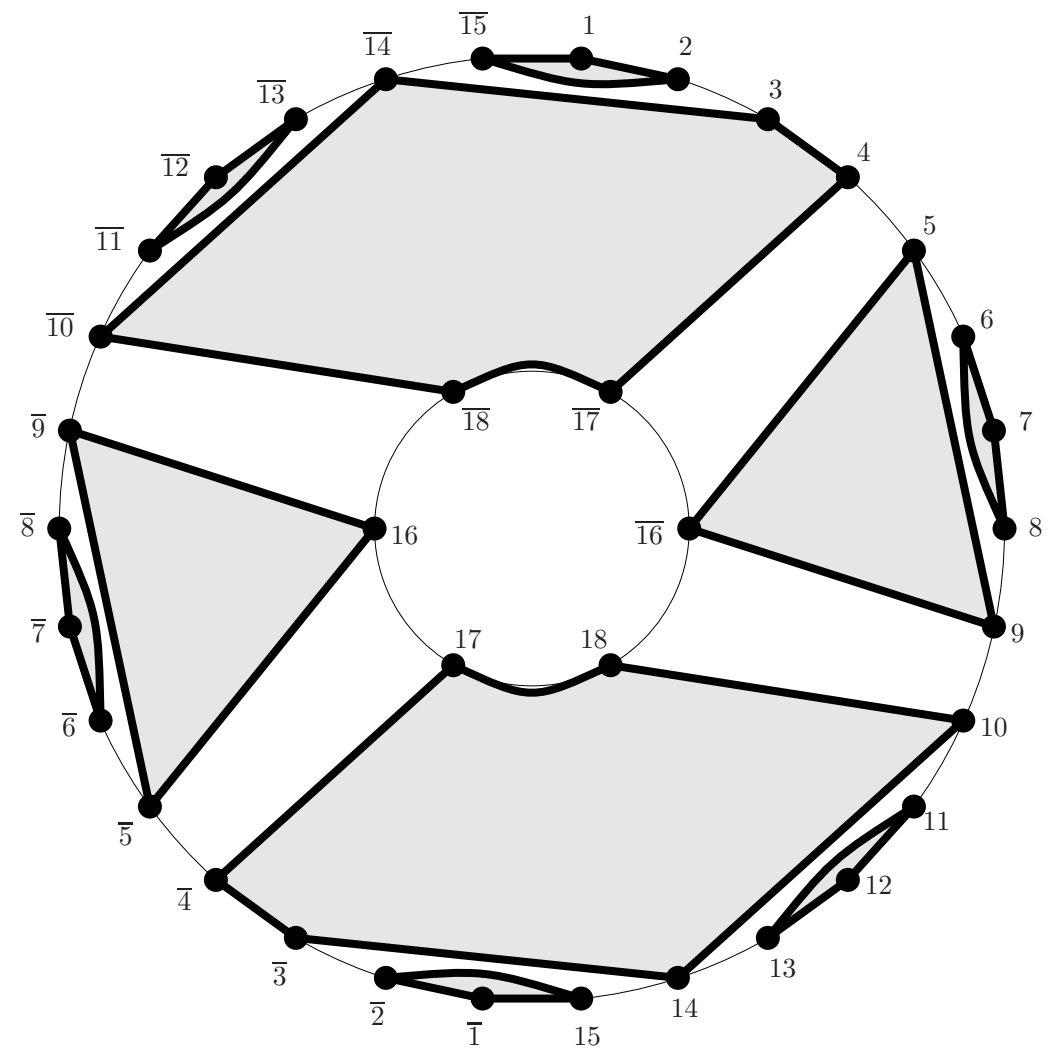

Figure 10. Combinatorial realisation of a 3-divisible non-crossing partition of type $D_{6}$

blocks come in "symmetric" pairs.) Consequently, under the bijection $\nabla_{B_{n}}^{m}$, the element $w_{0}$ contains a type $B$ cycle of length $\ell$ if and only if $\nabla_{B_{n}}^{m}\left(w_{0} ; w_{1}, \ldots, w_{m}\right)$ contains a zero block of size $m \ell$.

The $m$-divisible non-crossing partitions of type $D_{n}$ cannot be realised as certain "partitions non croisées d'un cycle," but as non-crossing partitions on an annulus with $2 m(n-1)$ vertices on the outer cycle and $2 m$ vertices on the inner cycle, with the vertices on the outer cycle being labelled by $1,2, \ldots, m n-m, \overline{1}, \overline{2}, \ldots, \overline{m n-m}$ in clockwise order and the vertices of the inner cycle being labelled by $m n-m+$ $1, \ldots, m n-1, m n, \overline{m n-m+1}, \ldots, \overline{m n-1}, \overline{m n}$ in counter-clockwise order. Given a partition $\pi$ of $\{1,2, \ldots, m n, \overline{1}, \overline{2}, \ldots, \overline{m n}\}$, we represent it on this annulus in a manner analogous to Kreweras' graphical representation of his partitions. Namely, we represent each block of $\pi$ by connecting the vertices labelled by the elements of the block by curves in clockwise order, the important additional requirement being here that the curves must be drawn in the interior of the annulus. If it is possible to draw the curves in such a way that no two curves intersect, then the partition is called a non-crossing partition on the $(2 m(n-1), 2 m)$-annulus. Figure 10 shows a non-crossing partition on the $(15,6)$-annulus.

With this definition, the $m$-divisible non-crossing partitions of type $D_{n}$ are in bijection with non-crossing partitions $\pi$ on the $(2 m(n-1), 2 m)$-annulus, in which successive elements of a block (successive in the circular order in the graphical 




FiguRE 11. A 3-divisible non-crossing partition of type $D_{6}$ with zero block

representation of the block) are in successive congruence classes modulo $m$, which have the property that, if $B$ is a block of $\pi$, then also $\bar{B}:=\{\bar{x}: x \in B\}$ is a block of $\pi$, and which satisfy an additional restriction concerning their zero block. Here again, a zero block is a block $B$ with $\bar{B}=B$. The announced additional restriction says that a zero block can only occur if it contains all the vertices of the inner cycle, that is, $m n-m+1, \ldots, m n-1, m n, \overline{m n-m+1}, \ldots, \overline{m n-1}, \overline{m n}$, and at least two further elements from the outer cycle. We denote this set of non-crossing partitions on the $(2 m(n-1), 2 m)$-annulus by $\widetilde{N C}^{m}\left(D_{n}\right)$. A non-crossing partition in $\widetilde{N C}^{m}\left(D_{n}\right)$ can only have at most one zero block. Figures 10 and11 give examples of 3-divisible non-crossing partitions of type $D_{6}$, with Figure 10 one without a zero block and Figure 11 one with a zero block. Again, it is clear that the condition that $B$ is a block of the partition if and only if $\bar{B}$ is a block translates into the condition that the geometric realisation of the partition is invariant under rotation by $180^{\circ}$.

In order to clearly sort out the differences of the earlier combinatorial realisations of $m$-divisible non-crossing partitions of types $A_{n-1}$ and $B_{n}$, we stress that for type $D_{n}$ there are three major features which are not present for the former types: (1) here we consider non-crossing partitions on an annulus; (2) it is not sufficient to impose the condition that the size of every block is divisible by $m$ : the condition on successive elements of a block is stronger; (3) there is the above additional restriction on the zero block (which is not present in type $B_{n}$ ). 
Given an element $\left(w_{0} ; w_{1}, \ldots, w_{m}\right) \in N C^{m}\left(D_{n}\right)$, the bijection, $\nabla_{D_{n}}^{m}$ say, from [29] works as follows. Recalling from Section 2 that $W\left(D_{n}\right)$ can be combinatorially realised as a subgroup of the group of permutations of $\{1,2, \ldots, n, \overline{1}, \overline{2}, \ldots, \bar{n}\}$ and that, in this realisation, the standard choice of a Coxeter element is $c=$ $[1,2, \ldots, n-1][n]=(1,2, \ldots, n-1, \overline{1}, \overline{2}, \ldots, \overline{n-1})(n, \bar{n})$, the announced bijection maps $\left(w_{0} ; w_{1}, \ldots, w_{m}\right) \in N C^{m}\left(D_{n}\right)$ to

$$
\begin{array}{r}
\nabla_{D_{n}}^{m}\left(w_{0} ; w_{1}, \ldots, w_{m}\right)=[1,2, \ldots, m(n-1)][m n-m+1, \ldots, m n-1, m n] \\
\circ\left(\bar{\tau}_{m, 1}\left(w_{1}\right)\right)^{-1}\left(\bar{\tau}_{m, 2}\left(w_{2}\right)\right)^{-1} \ldots\left(\bar{\tau}_{m, m}\left(w_{m}\right)\right)^{-1},
\end{array}
$$

where $\bar{\tau}_{m, i}$ is defined as above. We refer the reader to 29] for the details. For example, let $n=6, m=3, w_{0}=((2, \overline{4})), w_{1}=((2, \overline{6}))((4,5)), w_{2}=((1, \overline{5}))((2,3))$, and $w_{3}=((3,6))$. Then $\left(w_{0} ; w_{1}, w_{2}, w_{3}\right)$ is mapped to

$$
\begin{aligned}
& \nabla_{D_{6}}^{3}\left(w_{0} ; w_{1}, w_{2}, w_{3}\right) \\
& \quad=[1,2, \ldots, 15][16,17,18]((4, \overline{16}))((10,13))((2, \overline{14}))((5,8))((9,18)) \\
& \quad=((1,2, \overline{15}))((3,4, \overline{17}, \overline{18}, \overline{10}, \overline{14}))((5,9, \overline{16}))((6,7,8))((11,12,13)) .
\end{aligned}
$$

Figure 10 shows the graphical representation of (7.7).

It is shown in [29] that $\nabla_{D_{n}}^{m}$ is in fact an isomorphism between the posets $N C^{m}\left(D_{n}\right)$ and $\widetilde{N C}^{m}\left(D_{n}\right)$. Furthermore, it is proved in [29] that

$$
c_{i}\left(w_{0}\right)=b_{i}\left(\nabla_{D_{n}}^{m}\left(w_{0} ; w_{1}, \ldots, w_{m}\right)\right), \quad i=1,2, \ldots, n,
$$

where $c_{i}\left(w_{0}\right)$ denotes the number of type $A$ cycles of length $i$ of $w_{0}$ and $b_{i}(\pi)$ denotes one half of the number of non-zero blocks of size $m i$ in the non-crossing partition $\pi$. (Recall that non-zero blocks come in "symmetric" pairs.) Consequently, under the bijection $\nabla_{D_{n}}^{m}$, the element $w_{0}$ contains a type $D$ cycle of length $\ell$ if and only if $\nabla_{D_{n}}^{m}\left(w_{0} ; w_{1}, \ldots, w_{m}\right)$ contains a zero block of size $m \ell$.

\section{Decomposition numbers With FREE FACTORS, AND ENUMERATION IN THE POSET OF GENERALISED NON-CROSSING PARTITIONS}

This section is devoted to applying our formulae from Sections 46 for the decomposition numbers of the types $A_{n}, B_{n}$, and $D_{n}$ to the enumerative theory of generalised non-crossing partitions for these types. Theorems 11 15 present formulae for the number of minimal factorisations of Coxeter elements in types $A_{n}$, $B_{n}$, and $D_{n}$, respectively, where we do not prescribe the types of all the factors as for the decomposition numbers, but just for some of them, while we impose rank sum conditions on other factors. Immediate corollaries are formulae for the number of multi-chains $\pi_{1} \leq \pi_{2} \leq \cdots \leq \pi_{l-1}, l$ being given, in the posets $\widetilde{N C}\left(A_{n-1}\right)$, $\widetilde{N C}^{m}\left(B_{n}\right)$, and $\widetilde{N C} m\left(D_{n}\right)$, where the poset rank of $\pi_{i}$ equals $r_{i}$ and where the block structure of $\pi_{1}$ is prescribed; see Corollaries 12, 14, and 16. These results in turn imply all known enumerative results on ordinary and generalised non-crossing partitions via appropriate summations; see the remarks accompanying the corollaries. They also imply two further new results on chain enumeration in $\widetilde{N C}^{m}\left(D_{n}\right)$; see Corollaries 18 and 19 . We want to stress that, since $\widetilde{N C}^{m}(\Phi)$ and $N C^{m}(\Phi)$ are isomorphic as posets for $\Phi=A_{n-1}, B_{n}, D_{n}$, Corollaries [12, 14, 16, 17, and 18] imply obvious results for $N C^{m}(\Phi)$ in place of $\widetilde{N C}(\Phi), \Phi=A_{n-1}, B_{n}, D_{n}$, via (7.4), (7.6), respectively (7.8). 
We begin with our results for type $A_{n}$. The next theorem generalises Theorem 6 . which can be obtained from the former as the special case in which $l=1$ and $m_{1}=1$.

Theorem 11. For a positive integer $d$, let types $T_{1}, T_{2}, \ldots, T_{d}$ be given, where

$$
T_{i}=A_{1}^{m_{1}^{(i)}} * A_{2}^{m_{2}^{(i)}} * \cdots * A_{n}^{m_{n}^{(i)}}, \quad i=1,2, \ldots, d,
$$

and let $l, m_{1}, m_{2}, \ldots, m_{l}, s_{1}, s_{2}, \ldots, s_{l}$ be given non-negative integers with

$$
\operatorname{rk} T_{1}+\operatorname{rk} T_{2}+\cdots+\operatorname{rk} T_{d}+s_{1}+s_{2}+\cdots+s_{l}=n .
$$

Then the number of factorisations

$$
c=\sigma_{1} \sigma_{2} \cdots \sigma_{d} \sigma_{1}^{(1)} \sigma_{2}^{(1)} \cdots \sigma_{m_{1}}^{(1)} \sigma_{1}^{(2)} \sigma_{2}^{(2)} \cdots \sigma_{m_{2}}^{(2)} \cdots \sigma_{1}^{(l)} \sigma_{2}^{(l)} \cdots \sigma_{m_{l}}^{(l)},
$$

where $c$ is a Coxeter element in $W\left(A_{n}\right)$, such that the type of $\sigma_{i}$ is $T_{i}, i=1,2, \ldots, d$, and such that

$$
\ell_{T}\left(\sigma_{1}^{(i)}\right)+\ell_{T}\left(\sigma_{2}^{(i)}\right)+\cdots+\ell_{T}\left(\sigma_{m_{i}}^{(i)}\right)=s_{i}, \quad i=1,2, \ldots, l,
$$

is given by

$$
\begin{aligned}
&(n+1)^{d-1}\left(\prod_{i=1}^{d} \frac{1}{n-\mathrm{rk} T_{i}+1}\right.\left.\left(\begin{array}{c}
n-\mathrm{rk} T_{i}+1 \\
m_{1}^{(i)}, m_{2}^{(i)}, \ldots, m_{n}^{(i)}
\end{array}\right)\right) \\
& \times\left(\begin{array}{c}
m_{1}(n+1) \\
s_{1}
\end{array}\right)\left(\begin{array}{c}
m_{2}(n+1) \\
s_{2}
\end{array}\right) \ldots\left(\begin{array}{c}
m_{l}(n+1) \\
s_{l}
\end{array}\right),
\end{aligned}
$$

where the multinomial coefficient is defined as in Lemma 4.

Proof. In the factorisation (8.1), we first also fix the types of the $\sigma_{i}^{(j)}$ 's. For $i=$ $1,2, \ldots, m_{j}$ and $j=1,2, \ldots, l$, let the type of $\sigma_{i}^{(j)}$ be

$$
T_{i}^{(j)}=A_{1}^{m_{1}^{(i, j)}} * A_{2}^{m_{2}^{(i, j)}} * \cdots * A_{n}^{m_{n}^{(i, j)}} .
$$

We know that the number of these factorisations is given by (4.1), with $d$ replaced by $d+m_{1}+m_{2}+\cdots+m_{l}$ and the appropriate interpretations of the $m_{i}^{(j)}$ 's. Next we fix non-negative integers $r_{i}^{(j)}$ and sum the expression (4.1) over all possible types $T_{i}^{(j)}$ of rank $r_{i}^{(j)}, i=1,2, \ldots, m_{j}, j=1,2, \ldots, l$. The corresponding summations are completely analogous to the summation in the proof of Theorem 6 . As a result, we obtain

$$
\begin{aligned}
(n+1)^{d-1}\left(\prod_{i=1}^{d} \frac{1}{n-\mathrm{rk} T_{i}+1}\left(\begin{array}{c}
n-\mathrm{rk} T_{i}+1 \\
m_{1}^{(i)}, m_{2}^{(i)}, \ldots, m_{n}^{(i)}
\end{array}\right)\right) \\
\times\left(\begin{array}{c}
n+1 \\
r_{1}^{(1)}
\end{array}\right)\left(\begin{array}{c}
n+1 \\
r_{2}^{(1)}
\end{array}\right) \cdots\left(\begin{array}{c}
n+1 \\
r_{m_{1}}^{(1)}
\end{array}\right) \times\left(\begin{array}{c}
n+1 \\
r_{1}^{(2)}
\end{array}\right)\left(\begin{array}{c}
n+1 \\
r_{2}^{(2)}
\end{array}\right) \cdots\left(\begin{array}{c}
n+1 \\
r_{m_{2}}^{(2)}
\end{array}\right) \\
\times \cdots \times\left(\begin{array}{c}
n+1 \\
r_{1}^{(l)}
\end{array}\right)\left(\begin{array}{c}
n+1 \\
r_{2}^{(l)}
\end{array}\right) \cdots\left(\begin{array}{c}
n+1 \\
r_{m_{l}}^{(l)}
\end{array}\right)
\end{aligned}
$$

for the number of factorisations under consideration. In view of (8.2) and (2.4), to obtain the final result we must sum these expressions over all non-negative integers $r_{1}^{(1)}, \ldots, r_{m_{l}}^{(l)}$ satisfying the equations

$$
r_{1}^{(j)}+r_{2}^{(j)}+\cdots+r_{m_{j}}^{(j)}=s_{j}, \quad j=1,2, \ldots, l .
$$


This is easily done by means of the multivariate version of the Chu-Vandermonde summation. The formula in (8.3) follows.

In view of the combinatorial realisation of $m$-divisible non-crossing partitions of type $A_{n-1}$ which we described in Section 7 the special case $d=1$ of the above theorem has the following enumerative consequence.

Corollary 12. Let $l$ be a positive integer, and let $s_{1}, s_{2}, \ldots, s_{l}$ be non-negative integers with $s_{1}+s_{2}+\cdots+s_{l}=n-1$. The number of multi-chains $\pi_{1} \leq \pi_{2} \leq \cdots \leq$ $\pi_{l-1}$ in the poset $\widehat{N C}^{m}\left(A_{n-1}\right)$, with the property that $\operatorname{rk}\left(\pi_{i}\right)=s_{1}+s_{2}+\cdots+s_{i}$, $i=1,2, \ldots, l-1$, and that the number of blocks of size mi of $\pi_{1}$ is $b_{i}, i=1,2, \ldots, n$, is given by

$$
\frac{1}{b_{1}+b_{2}+\cdots+b_{n}}\left(\begin{array}{c}
b_{1}+b_{2}+\cdots+b_{n} \\
b_{1}, b_{2}, \ldots, b_{n}
\end{array}\right)\left(\begin{array}{c}
m n \\
s_{2}
\end{array}\right) \cdots\left(\begin{array}{c}
m n \\
s_{l}
\end{array}\right)
$$

provided that $b_{1}+2 b_{2}+\cdots+n b_{n} \leq n$, and is 0 otherwise.

Remark. The conditions in the statement of the corollary imply that

$$
s_{1}+b_{1}+b_{2}+\cdots+b_{n}=n \text {. }
$$

Proof. Let

$$
\pi_{1} \leq \pi_{2} \leq \cdots \leq \pi_{l-1}
$$

be a multi-chain in $\widetilde{N C}^{m}\left(A_{n-1}\right)$. Suppose that, under the bijection $\nabla_{A_{n-1}}^{m}$, the element $\pi_{j}$ corresponds to the tuple $\left(w_{0}^{(j)} ; w_{1}^{(j)}, \ldots, w_{m}^{(j)}\right), j=1,2, \ldots, l-1$. The inequalities in (8.7) imply that $w_{1}^{(1)}, w_{2}^{(1)}, \ldots, w_{m}^{(1)}$ can be factored in the form

$$
w_{i}^{(1)}=u_{i}^{(2)} u_{i}^{(3)} \cdots u_{i}^{(l)}, \quad i=1,2, \ldots, m,
$$

where $u_{i}^{(l)}=w_{i}^{(l-1)}$ and, more generally,

$$
w_{i}^{(j)}=u_{i}^{(j+1)} u_{i}^{(j+2)} \cdots u_{i}^{(l)}, \quad i=1,2, \ldots, m, j=1,2, \ldots, l-1 .
$$

For later use, we record that

$$
\begin{aligned}
c & =w_{0}^{(j)} w_{1}^{(j)} \cdots w_{m}^{(j)} \\
(8.9) \quad & =w_{0}^{(j)}\left(u_{1}^{(j+1)} u_{1}^{(j+2)} \cdots u_{1}^{(l)}\right)\left(u_{2}^{(j+1)} u_{2}^{(j+2)} \cdots u_{2}^{(l)}\right) \cdots\left(u_{m}^{(j+1)} u_{m}^{(j+2)} \cdots u_{m}^{(l)}\right) .
\end{aligned}
$$

Now, by (7.4), the block structure conditions on $\pi_{1}$ in the statement of the corollary translate into the condition that the type of $w_{0}^{(1)}$ is

$$
A_{1}^{b_{2}} * A_{2}^{b_{3}} * \cdots * A_{n-1}^{b_{n}} .
$$

On the other hand, using (17.2), we see that the rank conditions in the statement of the corollary mean that

$$
\ell_{T}\left(w_{0}^{(j)}\right)=s_{1}+s_{2}+\cdots+s_{j}, \quad j=1,2, \ldots, l-1 .
$$

In combination with (8.9), this yields the conditions

$$
\ell_{T}\left(u_{1}^{(j)}\right)+\ell_{T}\left(u_{2}^{(j)}\right)+\cdots+\ell_{T}\left(u_{m}^{(j)}\right)=s_{j}, \quad j=2,3, \ldots, l .
$$

Thus, we want to count the number of factorisations

$$
c=w_{0}^{(1)}\left(u_{1}^{(2)} u_{1}^{(3)} \cdots u_{1}^{(l)}\right)\left(u_{2}^{(2)} u_{2}^{(3)} \cdots u_{2}^{(l)}\right) \cdots\left(u_{m}^{(2)} u_{m}^{(3)} \cdots u_{m}^{(l)}\right),
$$


where the type of $w_{0}^{(1)}$ is given in (8.10), and where the "rank conditions" (8.11) are satisfied. So, in view of (2.4), we are in the situation of Theorem 11 with $n$ replaced by $n-1, d=1, l$ replaced by $l-1, s_{i}$ replaced by $s_{i+1}, i=1,2, \ldots, l-1$, $T_{1}$ the type in (8.10), $m_{1}=m_{2}=\cdots=m_{l-1}=m$, except that the factors are not exactly in the order as in (8.1). However, by (2.2) we know that the order of factors is without relevance. Therefore we just have to apply Theorem 11 with the above specialisations. If we also take (8.6) into account, then we immediately arrive at (8.5).

This result is new even for $m=1$, that is, for the poset of Kreweras' non-crossing partitions of $\{1,2, \ldots, n\}$. It implies all known results on Kreweras' non-crossing partitions and the $m$-divisible non-crossing partitions of Edelman. Namely, for $l=2$ it reduces to Armstrong's result [1, Theorem 4.4.4 with $\ell=1$ ] on the number of $m$-divisible non-crossing partitions in $\widetilde{N C}^{m}\left(A_{n-1}\right)$ with a given block structure, which itself contains Kreweras' result [30, Theorem 4] on his non-crossing partitions with a given block structure as a special case. If we sum the expression 8.5 over all $s_{2}, s_{3}, \ldots, s_{l}$ with $s_{2}+s_{3}+\cdots+s_{l}=n-1-s_{1}$, then we obtain that the number of all multi-chains $\pi_{1} \leq \pi_{2} \leq \cdots \leq \pi_{l-1}$ in Edelman's poset $\widetilde{N C}^{m}\left(A_{n-1}\right)$ of $m$ divisible non-crossing partitions of $\{1,2, \ldots, m n\}$ in which $\pi_{1}$ has $b_{i}$ blocks of size $m i$ equals

$$
\begin{aligned}
& \frac{1}{b_{1}+b_{2}+\cdots+b_{n}}\left(\begin{array}{c}
b_{1}+b_{2}+\cdots+b_{n} \\
b_{1}, b_{2}, \ldots, b_{n}
\end{array}\right)\left(\begin{array}{c}
(l-1) m n \\
n-s_{1}-1
\end{array}\right) \\
& =\frac{1}{b_{1}+b_{2}+\cdots+b_{n}}\left(\begin{array}{c}
b_{1}+b_{2}+\cdots+b_{n} \\
b_{1}, b_{2}, \ldots, b_{n}
\end{array}\right)\left(\begin{array}{c}
(l-1) m n \\
b_{1}+b_{2}+\cdots+b_{n}-1
\end{array}\right),
\end{aligned}
$$

provided that $b_{1}+2 b_{2}+\cdots+n b_{n} \leq n$, a result originally due to Armstrong [1, Theorem 4.4.4]. On the other hand, if we sum the expression 8.5 over all possible $b_{1}, b_{2}, \ldots, b_{n}$, that is, $b_{2}+2 b_{3}+\cdots+(n-1) b_{n}=s_{1}$, use of Lemma 4 with $M=n-s_{1}$ and $r=s_{1}$ yields that the number of all multi-chains $\pi_{1} \leq \pi_{2} \leq \cdots \leq \pi_{l-1}$ in Edelman's poset $\widetilde{N C^{m}}\left(A_{n-1}\right) \cong N C^{m}\left(A_{n-1}\right)$ where $\pi_{i}$ is of rank $s_{1}+s_{2}+\cdots+s_{i}$, $i=1,2, \ldots, l-1$, equals

$$
\frac{1}{n}\left(\begin{array}{c}
n \\
s_{1}
\end{array}\right)\left(\begin{array}{c}
m n \\
s_{2}
\end{array}\right) \cdots\left(\begin{array}{c}
m n \\
s_{l}
\end{array}\right),
$$

a result originally due to Edelman [18, Theorem 4.2]. Clearly, this formula contains at the same time a formula for the number of all $m$-divisible non-crossing partitions of $\{1,2, \ldots, m n\}$ with a given number of blocks upon setting $l=2$ (cf. [18, Lemma 4.1]), as well as implies that the total number of multi-chains $\pi_{1} \leq \pi_{2} \leq \cdots \leq \pi_{l-1}$ in the poset of these partitions is

$$
\frac{1}{n}\left(\begin{array}{c}
(l-1) m n+n \\
n-1
\end{array}\right)
$$

upon summing (8.14) over all non-negative integers $s_{1}, s_{2}, \ldots, s_{l}$ with $s_{1}+s_{2}+$ $\cdots+s_{l}=n-1$ by means of the multivariate Chu-Vandermonde summation, thus recovering the formula [18. Cor. 4.4] for the zeta polynomial of the poset of $m$ divisible non-crossing partitions of type $A_{n-1}$. As the special case $l=2$, we recover the well-known fact that the total number of $m$-divisible non-crossing partitions of $\{1,2, \ldots, m n\}$ is $\frac{1}{n}\left(\begin{array}{c}(m+1) n \\ n-1\end{array}\right)$. 
We continue with our results for type $B_{n}$. We formulate the theorem below on factorisations in $W\left(B_{n}\right)$ only with restrictions on the combinatorial type of some factors. An analogous result with group-theoretical type instead could be easily derived as well. We omit this here because, for the combinatorial applications that we have in mind, the combinatorial type suffices. We remark that the theorem generalises Theorem 8 , which can be obtained from the former as the special case in which $l=1$ and $m_{1}=1$.

Theorem 13. (i) For a positive integer $d$, let the types $T_{1}, T_{2}, \ldots, T_{d}$ be given, where

$$
T_{i}=A_{1}^{m_{1}^{(i)}} * A_{2}^{m_{2}^{(i)}} * \cdots * A_{n}^{m_{n}^{(i)}}, \quad i=1,2, \ldots, j-1, j+1, \ldots, d
$$

and

$$
T_{j}=B_{\alpha} * A_{1}^{m_{1}^{(j)}} * A_{2}^{m_{2}^{(j)}} * \cdots * A_{n}^{m_{n}^{(j)}}
$$

for some $\alpha \geq 1$, and let $l, m_{1}, m_{2}, \ldots, m_{l}, s_{1}, s_{2}, \ldots, s_{l}$ be given non-negative integers with

$$
\operatorname{rk} T_{1}+\operatorname{rk} T_{2}+\cdots+\operatorname{rk} T_{d}+s_{1}+s_{2}+\cdots+s_{l}=n .
$$

Then the number of factorisations

$$
c=\sigma_{1} \sigma_{2} \cdots \sigma_{d} \sigma_{1}^{(1)} \sigma_{2}^{(1)} \cdots \sigma_{m_{1}}^{(1)} \sigma_{1}^{(2)} \sigma_{2}^{(2)} \cdots \sigma_{m_{2}}^{(2)} \cdots \sigma_{1}^{(l)} \sigma_{2}^{(l)} \cdots \sigma_{m_{l}}^{(l)},
$$

where $c$ is a Coxeter element in $W\left(B_{n}\right)$, such that the combinatorial type of $\sigma_{i}$ is $T_{i}, i=1,2, \ldots, d$, and such that

$$
\ell_{T}\left(\sigma_{1}^{(i)}\right)+\ell_{T}\left(\sigma_{2}^{(i)}\right)+\cdots+\ell_{T}\left(\sigma_{m_{i}}^{(i)}\right)=s_{i}, \quad i=1,2, \ldots, l,
$$

is given by

$$
\begin{aligned}
n^{d-1}\left(\begin{array}{c}
n-\mathrm{rk} T_{j} \\
m_{1}^{(j)}, m_{2}^{(j)}, \ldots, m_{n}^{(j)}
\end{array}\right)\left(\prod_{\substack{i=1 \\
i \neq j}}^{d} \frac{1}{n-\operatorname{rk} T_{i}}\left(\begin{array}{c}
n-\operatorname{rk} T_{i} \\
\left.m_{1}^{(i)}, m_{2}^{(i)}, \ldots, m_{n}^{(i)}\right)
\end{array}\right)\right) \\
\\
\times\left(\begin{array}{c}
m_{1} n \\
s_{1}
\end{array}\right)\left(\begin{array}{c}
m_{2} n \\
s_{2}
\end{array}\right) \ldots\left(\begin{array}{c}
m_{l} n \\
s_{l}
\end{array}\right),
\end{aligned}
$$

where the multinomial coefficient is defined as in Lemma 4 .

(ii) For a positive integer $d$, let the types $T_{1}, T_{2}, \ldots, T_{d}$ be given, where

$$
T_{i}=A_{1}^{m_{1}^{(i)}} * A_{2}^{m_{2}^{(i)}} * \cdots * A_{n}^{m_{n}^{(i)}}, \quad i=1,2, \ldots, d,
$$

and let $l, m_{1}, m_{2}, \ldots, m_{l}, s_{1}, s_{2}, \ldots, s_{l}$ be given non-negative integers. Then the number of factorisations (8.17) which satisfy (8.18) plus the condition that the combinatorial type of $\sigma_{i}$ is $T_{i}, i=1,2, \ldots, d$, is given by

$$
\begin{array}{r}
n^{d-1}\left(n-\operatorname{rk} T_{1}-\operatorname{rk} T_{2}-\cdots-\operatorname{rk} T_{d}\right)\left(\prod_{i=1}^{d} \frac{1}{n-\operatorname{rk} T_{i}}\left(\begin{array}{c}
n-\operatorname{rk} T_{i} \\
m_{1}^{(i)}, m_{2}^{(i)}, \ldots, m_{n}^{(i)}
\end{array}\right)\right) \\
\times\left(\begin{array}{c}
m_{1} n \\
s_{1}
\end{array}\right)\left(\begin{array}{c}
m_{2} n \\
s_{2}
\end{array}\right) \cdots\left(\begin{array}{c}
m_{l} n \\
s_{l}
\end{array}\right) .
\end{array}
$$


Proof. We start with the proof of item (i). In the factorisation (8.17), we first also fix the types of $\sigma_{i}^{(j)}$ 's. For $i=1,2, \ldots, m_{j}$ and $j=1,2, \ldots, l$ let the type of $\sigma_{i}^{(j)}$ be

$$
T_{i}^{(j)}=A_{1}^{m_{1}^{(i, j)}} * A_{2}^{m_{2}^{(i, j)}} * \cdots * A_{n}^{m_{n}^{(i, j)}} .
$$

We know that the number of these factorisations is given by (5.1) with $d$ replaced by $d+m_{1}+m_{2}+\cdots+m_{l}$ and the appropriate interpretations of $m_{i}^{(j)}$ 's. Next we fix non-negative integers $r_{i}^{(j)}$ and sum the expression (5.1) over all possible types $T_{i}^{(j)}$ of $\operatorname{rank} r_{i}^{(j)}, i=1,2, \ldots, m_{j}, j=1,2, \ldots, l$. The corresponding summations are completely analogous to the first summation in the proof of Theorem 8 . As a result, we obtain

$$
\begin{array}{r}
n^{d-1}\left(\begin{array}{c}
n-\mathrm{rk} T_{j} \\
m_{1}^{(j)}, m_{2}^{(j)}, \ldots, m_{n}^{(j)}
\end{array}\right)\left(\prod_{\substack{i=1 \\
i \neq j}}^{d} \frac{1}{n-\mathrm{rk} T_{i}}\left(\begin{array}{c}
n-\mathrm{rk} T_{i} \\
m_{1}^{(i)}, m_{2}^{(i)}, \ldots, m_{n}^{(i)}
\end{array}\right)\right) \\
\times\left(\begin{array}{c}
n \\
r_{1}^{(1)}
\end{array}\right)\left(\begin{array}{c}
n \\
r_{2}^{(1)}
\end{array}\right) \cdots\left(\begin{array}{c}
n \\
r_{m_{1}}^{(1)}
\end{array}\right) \times\left(\begin{array}{c}
n \\
r_{1}^{(2)}
\end{array}\right)\left(\begin{array}{c}
n \\
r_{2}^{(2)}
\end{array}\right) \cdots\left(\begin{array}{c}
n \\
r_{m_{2}}^{(2)}
\end{array}\right) \\
\times \cdots \times\left(\begin{array}{c}
n \\
r_{1}^{(l)}
\end{array}\right)\left(\begin{array}{c}
n \\
r_{2}^{(l)}
\end{array}\right) \cdots\left(\begin{array}{c}
n \\
r_{m_{l}}^{(l)}
\end{array}\right)
\end{array}
$$

for the number of factorisations under consideration. In view of (8.18) and (2.4), to obtain the final result we must sum these expressions over all non-negative integers $r_{1}^{(1)}, \ldots, r_{m_{l}}^{(l)}$ satisfying the equations

$$
r_{1}^{(j)}+r_{2}^{(j)}+\cdots+r_{m_{j}}^{(j)}=s_{j}, \quad j=1,2, \ldots, l .
$$

This is easily done by means of the multivariate version of the Chu-Vandermonde summation. The formula in (8.19) follows.

The proof of item (ii) is completely analogous. We must, however, cope with the complication that the type $B$ cycle, which, according to Theorem 7 must occur in the disjoint cycle decomposition of exactly one of the factors on the right-hand side of (8.17), can occur in any of the $\sigma_{i}^{(j)}$ 's. So, let us fix the types of $\sigma_{i}^{(j)}$ 's to

$$
T_{i}^{(j)}=A_{1}^{m_{1}^{(i, j)}} * A_{2}^{m_{2}^{(i, j)}} * \cdots * A_{n}^{m_{n}^{(i, j)}},
$$

$i=1,2, \ldots, m_{j}, j=1,2, \ldots, l$, except for $(i, j)=(p, q)$, where we require that the type of $\sigma_{p}^{(q)}$ is

$$
T_{p}^{(q)}=B_{\alpha} * A_{1}^{\widetilde{m}_{1}} * A_{2}^{\widetilde{m}_{2}} * \cdots * A_{n}^{\widetilde{m}_{n}} .
$$

Again, we know that the number of these factorisations is given by (5.1), with $d$ replaced by $d+m_{1}+m_{2}+\cdots+m_{l}$ and the appropriate interpretations of the $m_{i}^{(j)}$ 's. Now we fix non-negative integers $r_{i}^{(j)}$ and sum the expression (5.1) over all possible types $T_{i}^{(j)}$ of $\operatorname{rank} r_{i}^{(j)}, i=1,2, \ldots, m_{j}, j=1,2, \ldots, l$. Again, the corresponding summations are completely analogous to the summations in the proof of Theorem 8. In particular, the summation over all possible types $T_{p}^{(q)}$ of rank $r_{p}^{(q)}$ is essentially the summation on the right-hand side of (5.14), with $d$ replaced by $d+m_{1}+m_{2}+\cdots+m_{l}$ and $r$ replaced by $r_{p}^{(q)}$. If we use what we know from 
the proof of Theorem 8 then the result of the summations is found to be

$$
\begin{aligned}
n^{d-1}\left(\prod_{i=1}^{d} \frac{1}{n-\operatorname{rk} T_{i}}\left(\begin{array}{c}
n-\operatorname{rk} T_{i} \\
m_{1}^{(i)}, m_{2}^{(i)}, \ldots, m_{n}^{(i)}
\end{array}\right)\right) \\
\times\left(\begin{array}{c}
n \\
r_{1}^{(1)}
\end{array}\right)\left(\begin{array}{c}
n \\
r_{2}^{(1)}
\end{array}\right) \cdots\left(\begin{array}{c}
n \\
r_{m_{1}}^{(1)}
\end{array}\right) \times \cdots \times\left(\begin{array}{c}
n \\
r_{1}^{(q)}
\end{array}\right) \cdots r_{p}^{(q)}\left(\begin{array}{c}
n \\
r_{p}^{(q)}
\end{array}\right) \cdots\left(\begin{array}{c}
n \\
r_{m_{q}}^{(q)}
\end{array}\right) \\
\times \cdots \times\left(\begin{array}{c}
n \\
r_{1}^{(l)}
\end{array}\right)\left(\begin{array}{c}
n \\
r_{2}^{(l)}
\end{array}\right) \cdots\left(\begin{array}{c}
n \\
r_{m_{l}}^{(l)}
\end{array}\right) .
\end{aligned}
$$

The reader should note that the term $r_{p}^{(q)}\left(\underset{r_{p}^{(q)}}{n}\right)$ in this expression results from the summation over all types $T_{p}^{(q)}$ of rank $r_{p}^{(q)}$ (compare (5.15) with $r$ replaced by $r_{p}^{(q)}$; we have $\left.\left(\begin{array}{c}n-1 \\ r_{p}^{(q)}-1\end{array}\right)=\frac{r_{p}^{(q)}}{n}\left(\begin{array}{c}n \\ r_{p}^{(q)}\end{array}\right)\right)$. Using (8.16),$($ 8.18) ) and (2.4), we see that the sum of all $r_{p}^{(q)}$ over $p=1,2, \ldots, m_{q}$ and $q=1,2, \ldots, l$ must be $n-\operatorname{rk} T_{1}-\operatorname{rk} T_{2}-\cdots-\operatorname{rk} T_{d}$. Hence, the sum of the expressions (8.21) over all $(p, q)$ equals

$$
\begin{array}{r}
n^{d-1}\left(n-\operatorname{rk} T_{1}-\operatorname{rk} T_{2}-\cdots-\operatorname{rk} T_{d}\right)\left(\prod_{i=1}^{d} \frac{1}{n-\operatorname{rk} T_{i}}\left(\begin{array}{c}
n-\operatorname{rk} T_{i} \\
m_{1}^{(i)}, m_{2}^{(i)}, \ldots, m_{n}^{(i)}
\end{array}\right)\right) \\
\times\left(\begin{array}{c}
n \\
r_{1}^{(1)}
\end{array}\right)\left(\begin{array}{c}
n \\
r_{2}^{(1)}
\end{array}\right) \cdots\left(\begin{array}{c}
n \\
r_{m_{1}}^{(1)}
\end{array}\right) \times \cdots \times\left(\begin{array}{c}
n \\
r_{1}^{(q)}
\end{array}\right)\left(\begin{array}{c}
n \\
r_{2}^{(q)}
\end{array}\right) \cdots\left(\begin{array}{c}
n \\
r_{m_{q}}^{(q)}
\end{array}\right) \\
\times \cdots \times\left(\begin{array}{c}
n \\
r_{1}^{(l)}
\end{array}\right)\left(\begin{array}{c}
n \\
r_{2}^{(l)}
\end{array}\right) \cdots\left(\begin{array}{c}
n \\
r_{m_{l}}^{(l)}
\end{array}\right) .
\end{array}
$$

Finally, we must sum these expressions over all non-negative integers $r_{1}^{(1)}, \ldots, r_{m_{l}}^{(l)}$ satisfying the equations

$$
r_{1}^{(j)}+r_{2}^{(j)}+\cdots+r_{m_{j}}^{(j)}=s_{j}, \quad j=1,2, \ldots, l .
$$

Once again, this is easily done by means of the multivariate version of the ChuVandermonde summation. As a result, we obtain the formula in (8.20).

In view of the combinatorial realisation of $m$-divisible non-crossing partitions of type $B_{n}$ which we described in Section 7 the special case $d=1$ of the above theorem has the following enumerative consequence.

Corollary 14. Let $l$ be a positive integer, and let $s_{1}, s_{2}, \ldots, s_{l}$ be non-negative integers with $s_{1}+s_{2}+\cdots+s_{l}=n$. The number of multi-chains $\pi_{1} \leq \pi_{2} \leq \cdots \leq$ $\pi_{l-1}$ in the poset $\widetilde{N C}^{m}\left(B_{n}\right)$ with the property that $\operatorname{rk}\left(\pi_{i}\right)=s_{1}+s_{2}+\cdots+s_{i}$, $i=1,2, \ldots, l-1$, and that the number of non-zero blocks of $\pi_{1}$ of size mi is $2 b_{i}$, $i=1,2, \ldots, n$, is given by

$$
\left(\begin{array}{c}
b_{1}+b_{2}+\cdots+b_{n} \\
b_{1}, b_{2}, \ldots, b_{n}
\end{array}\right)\left(\begin{array}{c}
m n \\
s_{2}
\end{array}\right) \ldots\left(\begin{array}{c}
m n \\
s_{l}
\end{array}\right),
$$

provided that $b_{1}+2 b_{2}+\cdots+n b_{n} \leq n$, and is 0 otherwise.

Remark. The conditions in the statement of the corollary imply that

$$
s_{1}+b_{1}+b_{2}+\cdots+b_{n}=n .
$$

The reader should recall from Section 7 that non-zero blocks of elements $\pi$ of $\widetilde{N C}^{m}\left(B_{n}\right)$ occur in pairs since, with a block $B$ of $\pi, \bar{B}$ also is a block of $\pi$. 
Proof. The arguments are completely analogous to those of the proof of Corollary 12. The conclusion here is that we need Theorem 13 with $d=1, l$ replaced by $l-1, s_{i}$ replaced by $s_{i+1}, i=1,2, \ldots, l-1, m_{1}=m_{2}=\cdots=m_{l-1}=m$, and $T_{1}$ of the type

$$
B_{n-b_{1}-2 b_{2}-\cdots-n b_{n}} * A_{1}^{b_{2}} * A_{2}^{b_{3}} * \cdots * A_{n-1}^{b_{n}}
$$

in the case that $b_{1}+2 b_{2}+\cdots+n b_{n}<n$ (which enforces the existence of a zero block of size $2\left(n-b_{1}-2 b_{2}-\cdots-n b_{n}\right)$ in $\left.\pi_{1}\right)$, respectively

$$
A_{1}^{b_{2}} * A_{2}^{b_{3}} * \cdots * A_{n-1}^{b_{n}}
$$

if not. So, depending on the case which we are in, we have to apply (8.19), respectively (8.20). However, for $d=1$ these two formulae become identical. More precisely, under the above specialisations, they reduce to

$$
\left(\begin{array}{c}
n-\mathrm{rk} T_{1} \\
b_{2}, b_{3}, \ldots, b_{n}
\end{array}\right)\left(\begin{array}{c}
m n \\
s_{2}
\end{array}\right) \ldots\left(\begin{array}{c}
m n \\
s_{l}
\end{array}\right)
$$

If we also take into account (8.23), then we arrive immediately at (8.22).

This result is new even for $m=1$, that is, for the poset of Reiner's type $B_{n}$ noncrossing partitions. It implies all known results on these non-crossing partitions and their extension to $m$-divisible type $B_{n}$ non-crossing partitions due to Armstrong. Namely, for $l=2$ it reduces to Armstrong's result [1, Theorem 4.5.11 with $\ell=1$ ] on the number of elements of $\widetilde{N C}^{m}\left(B_{n}\right)$ with a given block structure, which itself contains Athanasiadis' result [2, Theorem 2.3] on Reiner's type $B_{n}$ non-crossing partitions with a given block structure as a special case. If we sum the expression (8.22) over all $s_{2}, s_{3}, \ldots, s_{l}$ with $s_{2}+s_{3}+\cdots+s_{l}=n-s_{1}$, then we obtain that the number of all multi-chains $\pi_{1} \leq \pi_{2} \leq \cdots \leq \pi_{l-1}$ in $\widetilde{N C^{m}}\left(B_{n}\right)$ in which $\pi_{1}$ has $2 b_{i}$ non-zero blocks of size $m i$ equals

$$
\left(\begin{array}{c}
b_{1}+b_{2}+\cdots+b_{n} \\
b_{1}, b_{2}, \ldots, b_{n}
\end{array}\right)\left(\begin{array}{c}
(l-1) m n \\
n-s_{1}
\end{array}\right)=\left(\begin{array}{c}
b_{1}+b_{2}+\cdots+b_{n} \\
b_{1}, b_{2}, \ldots, b_{n}
\end{array}\right)\left(\begin{array}{c}
(l-1) m n \\
b_{1}+b_{2}+\cdots+b_{n}
\end{array}\right)
$$

provided that $b_{1}+2 b_{2}+\cdots+n b_{n} \leq n$, a result originally due to Armstrong [1, Theorem 4.5.11]. On the other hand, if we sum the expression 8.22) over all possible $b_{1}, b_{2}, \ldots, b_{n}$, that is, over $b_{2}+2 b_{3}+\cdots+(n-1) b_{n} \leq s_{1}$, use of Lemma 4 with $M=n-s_{1}$ and $r=s_{1}-\alpha$ (where $\alpha$ stands for the difference between $s_{1}$ and $\left.b_{2}+2 b_{3}+\cdots+(n-1) b_{n}\right)$ yields that the number of all multi-chains $\pi_{1} \leq$ $\pi_{2} \leq \cdots \leq \pi_{l-1}$ in $\widetilde{N C^{m}}\left(B_{n}\right) \cong N C^{m}\left(B_{n}\right)$, where $\pi_{i}$ is of rank $s_{1}+s_{2}+\cdots+s_{i}$, $i=1,2, \ldots, l-1$, equals

$$
\sum_{\alpha=0}^{n}\left(\begin{array}{c}
n-\alpha-1 \\
s_{1}-\alpha
\end{array}\right)\left(\begin{array}{c}
m n \\
s_{2}
\end{array}\right) \cdots\left(\begin{array}{c}
m n \\
s_{l}
\end{array}\right)=\left(\begin{array}{c}
n \\
s_{1}
\end{array}\right)\left(\begin{array}{c}
m n \\
s_{2}
\end{array}\right) \cdots\left(\begin{array}{c}
m n \\
s_{l}
\end{array}\right),
$$

another result due to Armstrong [1, Theorem 4.5.7]. Clearly, this formula contains at the same time a formula for the number of all elements of $\widetilde{N C}^{m}\left(B_{n}\right) \cong N C^{m}\left(B_{n}\right)$ with a given number of blocks (equivalently, a given rank) upon setting $l=2$ (cf. [1, Theorem 4.5.8]), as well as implies that the total number of multi-chains $\pi_{1} \leq \pi_{2} \leq \cdots \leq \pi_{l-1}$ in $\widetilde{N C}^{m}\left(B_{n}\right) \cong N C^{m}\left(B_{n}\right)$ is

$$
\left(\begin{array}{c}
(l-1) m n+n \\
n
\end{array}\right)
$$


upon summing 8.25) over all non-negative integers $s_{1}, s_{2}, \ldots, s_{l}$ with $s_{1}+s_{2}+$ $\cdots+s_{l}=n$ by means of the multivariate $\mathrm{Chu}$-Vandermonde summation, thus recovering the formula [1, Theorem 3.6.9] for the zeta polynomial of the poset of generalised non-crossing partitions in the case of type $B_{n}$. As the special case $l=2$, we recover the fact that the cardinality of $\widetilde{N C}^{m}\left(B_{n}\right) \cong N C^{m}\left(B_{n}\right)$ is $\left(\begin{array}{c}(m+1) n \\ n\end{array}\right)$ (cf. 11. Theorem 3.5.3]).

The final set of results in this section concerns type $D_{n}$. We start with Theorem 15. the result on factorisations in $W\left(D_{n}\right)$, which is analogous to Theorems 11 and 13. Similar to Theorem[13. we formulate the theorem only with restrictions on the combinatorial type of some factors. An analogous result with group-theoretical type instead could be easily derived as well. We refrain from doing this here because, again, for the combinatorial applications that we have in mind, combinatorial type suffices. We remark that the theorem generalises Theorem 10, which can be obtained from the former as the special case in which $l=1$ and $m_{1}=1$.

Theorem 15. (i) For a positive integer $d$, let the types $T_{1}, T_{2}, \ldots, T_{d}$ be given, where

$$
T_{i}=A_{1}^{m_{1}^{(i)}} * A_{2}^{m_{2}^{(i)}} * \cdots * A_{n}^{m_{n}^{(i)}}, \quad i=1,2, \ldots, j-1, j+1, \ldots, d
$$

and

$$
T_{j}=D_{\alpha} * A_{1}^{m_{1}^{(j)}} * A_{2}^{m_{2}^{(j)}} * \cdots * A_{n}^{m_{n}^{(j)}},
$$

for some $\alpha \geq 2$, and let $l, m_{1}, m_{2}, \ldots, m_{l}, s_{1}, s_{2}, \ldots, s_{l}$ be given non-negative integers with

$$
\operatorname{rk} T_{1}+\operatorname{rk} T_{2}+\cdots+\operatorname{rk} T_{d}+s_{1}+s_{2}+\cdots+s_{l}=n .
$$

Then the number of factorisations

$$
c=\sigma_{1} \sigma_{2} \cdots \sigma_{d} \sigma_{1}^{(1)} \sigma_{2}^{(1)} \cdots \sigma_{m_{1}}^{(1)} \sigma_{1}^{(2)} \sigma_{2}^{(2)} \cdots \sigma_{m_{2}}^{(2)} \cdots \sigma_{1}^{(l)} \sigma_{2}^{(l)} \cdots \sigma_{m_{l}}^{(l)},
$$

where $c$ is a Coxeter element in $W\left(D_{n}\right)$, such that the combinatorial type of $\sigma_{i}$ is $T_{i}, i=1,2, \ldots, d$, and such that

$$
\ell_{T}\left(\sigma_{1}^{(i)}\right)+\ell_{T}\left(\sigma_{2}^{(i)}\right)+\cdots+\ell_{T}\left(\sigma_{m_{i}}^{(i)}\right)=s_{i}, \quad i=1,2, \ldots, l,
$$

is given by

$$
\begin{aligned}
(n-1)^{d-1}\left(\begin{array}{c}
n-\mathrm{rk} T_{j} \\
m_{1}^{(j)}, m_{2}^{(j)}, \ldots, m_{n}^{(j)}
\end{array}\right) & \left(\prod_{\substack{i=1 \\
i \neq j}}^{d} \frac{1}{n-\mathrm{rk} T_{i}-1}\left(\begin{array}{c}
n-\operatorname{rk} T_{i}-1 \\
m_{1}^{(i)}, m_{2}^{(i)}, \ldots, m_{n}^{(i)}
\end{array}\right)\right) \\
& \times\left(\begin{array}{c}
m_{1}(n-1) \\
s_{1}
\end{array}\right)\left(\begin{array}{c}
m_{2}(n-1) \\
s_{2}
\end{array}\right) \ldots\left(\begin{array}{c}
m_{l}(n-1) \\
s_{l}
\end{array}\right),
\end{aligned}
$$

the multinomial coefficient being defined as in Lemma 4 .

(ii) For a positive integer $d$, let the types $T_{1}, T_{2}, \ldots, T_{d}$ be given, where

$$
T_{i}=A_{1}^{m_{1}^{(i)}} * A_{2}^{m_{2}^{(i)}} * \cdots * A_{n}^{m_{n}^{(i)}}, \quad i=1,2, \ldots, d,
$$


and let $l, m_{1}, m_{2}, \ldots, m_{l}, s_{1}, s_{2}, \ldots, s_{l}$ be given non-negative integers. Then the number of factorisations (8.27) which satisfy (8.28) as well as the condition that the combinatorial type of $\sigma_{i}$ is $T_{i}, i=1,2, \ldots, d$, is given by

$$
\begin{aligned}
& 2(n-1)^{d-1}\left(\sum_{j=1}^{d}\left(\begin{array}{c}
n-\operatorname{rk} T_{j} \\
m_{1}^{(j)}, m_{2}^{(j)}, \ldots, m_{n}^{(j)}
\end{array}\right) \prod_{\substack{i=1 \\
i \neq j}}^{d} \frac{1}{n-\operatorname{rk} T_{i}-1}\left(\begin{array}{c}
n-\operatorname{rk} T_{i}-1 \\
m_{1}^{(i)}, m_{2}^{(i)}, \ldots, m_{n}^{(i)}
\end{array}\right)\right) \\
& \times\left(\begin{array}{c}
m_{1}(n-1) \\
s_{1}
\end{array}\right)\left(\begin{array}{c}
m_{2}(n-1) \\
s_{2}
\end{array}\right) \cdots\left(\begin{array}{c}
m_{l}(n-1) \\
s_{l}
\end{array}\right) \\
& +(n-1)^{d}\left(\prod_{i=1}^{d} \frac{1}{n-\mathrm{rk} T_{i}-1}\left(\begin{array}{c}
n-\mathrm{rk} T_{i}-1 \\
m_{1}^{(i)}, m_{2}^{(i)}, \ldots, m_{n}^{(i)}
\end{array}\right)\right) \\
& \times \sum_{j=1}^{l} m_{j}\left(\begin{array}{c}
m_{1}(n-1) \\
s_{1}
\end{array}\right) \cdots\left(\begin{array}{c}
m_{j}(n-1)-1 \\
s_{j}-2
\end{array}\right) \cdots\left(\begin{array}{c}
m_{l}(n-1) \\
s_{l}
\end{array}\right) \\
& -2(d-1)(n-1)^{d}\left(\prod_{i=1}^{d} \frac{1}{n-\operatorname{rk} T_{i}-1}\left(\begin{array}{c}
n-\operatorname{rk} T_{i}-1 \\
m_{1}^{(i)}, m_{2}^{(i)}, \ldots, m_{n}^{(i)}
\end{array}\right)\right) \\
& \times\left(\begin{array}{c}
m_{1}(n-1) \\
s_{1}
\end{array}\right)\left(\begin{array}{c}
m_{2}(n-1) \\
s_{2}
\end{array}\right) \cdots\left(\begin{array}{c}
m_{l}(n-1) \\
s_{l}
\end{array}\right) .
\end{aligned}
$$

Proof. The proof of item (i) is completely analogous to the proof of item (i) in Theorem 13. Making reference to that proof, the only difference is that, instead of the expression (5.1), we must use (6.1) with $d$ replaced by $d+m_{1}+m_{2}+\cdots+m_{l}$ and the appropriate interpretations of the $m_{i}^{(j)}$ 's. The summations over types $T_{i}^{(j)}$ with fixed rank $r_{i}^{(j)}$ are carried out by using (3.4) with $M=n-r-1$. Subsequently, the summations over the $r_{i}^{(j)}$ 's satisfying (8.4) are done by the multivariate version of the $\mathrm{Chu}$-Vandermonde summation. We leave it to the reader to fill in the details to finally arrive at 8.29 .

Similarly, the proof of item (ii) is analogous to the proof of item (ii) in Theorem [13. However, we must cope with the complication that there may or may not be a type $D$ cycle in the disjoint cycle decomposition of one of the $\sigma_{i}^{(j)}$ 's on the right-hand side of (8.27). In the case that there is no type $B$ cycle, we fix the types of the $\sigma_{i}^{(j)}$ 's to

$$
T_{i}^{(j)}=A_{1}^{m_{1}^{(i, j)}} * A_{2}^{m_{2}^{(i, j)}} * \cdots * A_{n}^{m_{n}^{(i, j)}}
$$

$i=1,2, \ldots, m_{j}, j=1,2, \ldots, l$, and sum the expression 6.2 with $d$ replaced by $d+m_{1}+m_{2}+\cdots+m_{l}$ and the appropriate interpretations of the $m_{i}^{(j)}$ 's over all possible types $T_{i}^{(j)}$ with $\operatorname{rank} r_{i}^{(j)}, i=1,2, \ldots, m_{j}, j=1,2, \ldots, l$. This yields the 
expression

$$
\begin{aligned}
2(n-1)^{d-1} & \left(\sum_{j=1}^{d}\left(\begin{array}{c}
n-\mathrm{rk} T_{j} \\
m_{1}^{(j)}, m_{2}^{(j)}, \ldots, m_{n}^{(j)}
\end{array}\right) \prod_{\substack{i=1 \\
i \neq j}}^{d} \frac{1}{n-\operatorname{rk} T_{i}-1}\left(\begin{array}{c}
n-\mathrm{rk} T_{i}-1 \\
m_{1}^{(i)}, m_{2}^{(i)}, \ldots, m_{n}^{(i)}
\end{array}\right)\right) \\
\times & \left(\begin{array}{c}
n-1 \\
r_{1}^{(1)}
\end{array}\right)\left(\begin{array}{c}
n-1 \\
r_{2}^{(1)}
\end{array}\right) \cdots\left(\begin{array}{c}
n-1 \\
r_{m_{1}}^{(1)}
\end{array}\right) \times \cdots \times\left(\begin{array}{c}
n-1 \\
r_{1}^{(l)}
\end{array}\right)\left(\begin{array}{c}
n-1 \\
r_{2}^{(l)}
\end{array}\right) \ldots\left(\begin{array}{c}
n-1 \\
r_{m_{l}}^{(l)}
\end{array}\right) \\
& +2(n-1)^{d}\left(\sum_{j=1}^{l} \sum_{i=1}^{m_{j}} \prod_{i=1}^{d} \frac{1}{n-\operatorname{rk} T_{i}-1}\left(\begin{array}{c}
n-\operatorname{rk} T_{i}-1 \\
m_{1}^{(i)}, m_{2}^{(i)}, \ldots, m_{n}^{(i)}
\end{array}\right)\right) \\
\times & \left(\begin{array}{c}
n-1 \\
r_{1}^{(1)}
\end{array}\right)\left(\begin{array}{c}
n-1 \\
r_{2}^{(1)}
\end{array}\right) \ldots\left(\begin{array}{c}
n-1 \\
r_{m_{1}}^{(1)}
\end{array}\right) \times \cdots \times\left(\begin{array}{c}
n-1 \\
r_{1}^{(l)}
\end{array}\right)\left(\begin{array}{c}
n-1 \\
r_{2}^{(l)}
\end{array}\right) \cdots\left(\begin{array}{c}
n-1 \\
r_{m_{l}}^{(l)}
\end{array}\right) \\
& -2(d-1)(n-1)^{d-1}\left(\prod_{i=1}^{d} \frac{1}{n-\operatorname{rk} T_{i}-1}\left(\begin{array}{c}
n-\operatorname{rk} T_{i}-1 \\
m_{1}^{(i)}, m_{2}^{(i)}, \ldots, m_{n}^{(i)}
\end{array}\right)\right) \\
& \times\left(\begin{array}{c}
n-1 \\
r_{1}^{(1)}
\end{array}\right)\left(\begin{array}{c}
n-1 \\
r_{2}^{(1)}
\end{array}\right) \cdots\left(\begin{array}{c}
n-1 \\
r_{m_{1}}^{(1)}
\end{array}\right) \times \cdots \times\left(\begin{array}{c}
n-1 \\
r_{1}^{(l)}
\end{array}\right)\left(\begin{array}{c}
n-1 \\
r_{2}^{(l)}
\end{array}\right) \cdots\left(\begin{array}{c}
n-1 \\
r_{m_{l}}^{(l)}
\end{array}\right) .
\end{aligned}
$$

In the case that there appears, however, a type $B$ cycle in $\sigma_{p}^{(q)}$, say, we adopt the same set-up as above, except that we restrict $\sigma_{p}^{(q)}$ to types of the form

$$
T_{p}^{(q)}=D_{\alpha} * A_{1}^{\widetilde{m}_{1}} * A_{2}^{\widetilde{m}_{2}} * \cdots * A_{n}^{\widetilde{m}_{n}}
$$

Subsequently, we sum the expression (6.1) with $d$ replaced by $d+m_{1}+m_{2}+\cdots+m_{l}$ and the appropriate interpretations of the $m_{i}^{(j)}$ 's over all possible types $T_{i}^{(j)}$ of rank $r_{i}^{(j)}$. This time, we obtain

$$
\begin{array}{r}
(n-1)^{d}\left(\sum_{q=1}^{l} \sum_{p=1}^{m_{q}} \sum_{\alpha=2}^{n} \prod_{i=1}^{d} \frac{1}{n-\operatorname{rk} T_{i}-1}\left(\begin{array}{c}
n-\operatorname{rk} T_{i}-1 \\
m_{1}^{(i)}, m_{2}^{(i)}, \ldots, m_{n}^{(i)}
\end{array}\right)\right) \\
\times\left(\begin{array}{c}
n-1 \\
r_{1}^{(1)}
\end{array}\right)\left(\begin{array}{c}
n-1 \\
r_{2}^{(1)}
\end{array}\right) \cdots\left(\begin{array}{c}
n-1 \\
r_{m_{1}}^{(1)}
\end{array}\right) \times \cdots \times\left(\begin{array}{c}
n-1 \\
r_{1}^{(q)}
\end{array}\right) \cdots\left(\begin{array}{c}
n-\alpha-1 \\
r_{p}^{(q)}-\alpha
\end{array}\right) \cdots\left(\begin{array}{c}
n-1 \\
r_{m_{q}}^{(q)}
\end{array}\right) \\
\times \cdots \times\left(\begin{array}{c}
n-1 \\
r_{1}^{(l)}
\end{array}\right)\left(\begin{array}{c}
n-1 \\
r_{2}^{(l)}
\end{array}\right) \cdots\left(\begin{array}{c}
n-1 \\
r_{m_{l}}^{(l)}
\end{array}\right) .
\end{array}
$$

The sum over $\alpha$ can be evaluated by means of the elementary summation formula

$$
\sum_{\alpha=2}^{n}\left(\begin{array}{c}
n-\alpha-1 \\
r-\alpha
\end{array}\right)=\sum_{\alpha=2}^{n}\left(\begin{array}{c}
n-\alpha-1 \\
n-r-1
\end{array}\right)=\left(\begin{array}{l}
n-2 \\
n-r
\end{array}\right)=\left(\begin{array}{l}
n-2 \\
r-2
\end{array}\right) .
$$

Finally, we must sum the expressions (8.31) and (8.32) over all non-negative integers $r_{1}^{(1)}, \ldots, r_{m_{l}}^{(l)}$ satisfying the equations

$$
r_{1}^{(j)}+r_{2}^{(j)}+\cdots+r_{m_{j}}^{(j)}=s_{j}, \quad j=1,2, \ldots, l .
$$

Once again, this is easily done by means of the multivariate version of the ChuVandermonde summation. After some simplification, we obtain the formula in (8.30). 
In view of the combinatorial realisation of $m$-divisible non-crossing partitions of type $D_{n}$ which we described in Section 7 the special case $d=1$ of the above theorem has the following enumerative consequence.

Corollary 16. Let $l$ be a positive integer, and let $s_{1}, s_{2}, \ldots, s_{l}$ be non-negative integers with $s_{1}+s_{2}+\cdots+s_{l}=n$. The number of multi-chains $\pi_{1} \leq \pi_{2} \leq \cdots \leq$ $\pi_{l-1}$ in the poset $\widehat{N C} C^{m}\left(D_{n}\right)$ with the property that $\operatorname{rk}\left(\pi_{i}\right)=s_{1}+s_{2}+\cdots+s_{i}$, $i=1,2, \ldots, l-1$, and that the number of non-zero blocks of $\pi_{1}$ of size mi is $2 b_{i}$, $i=1,2, \ldots, n$, is given by

$$
\left(\begin{array}{c}
b_{1}+b_{2}+\cdots+b_{n} \\
b_{1}, b_{2}, \ldots, b_{n}
\end{array}\right)\left(\begin{array}{c}
m(n-1) \\
s_{2}
\end{array}\right) \cdots\left(\begin{array}{c}
m(n-1) \\
s_{l}
\end{array}\right)
$$

if $b_{1}+2 b_{2}+\cdots+n b_{n}<n-1$, and

$$
\begin{aligned}
& (8.34) 2\left(\begin{array}{c}
b_{1}+b_{2}+\cdots+b_{n} \\
b_{1}, b_{2}, \ldots, b_{n}
\end{array}\right)\left(\begin{array}{c}
m(n-1) \\
s_{2}
\end{array}\right) \cdots\left(\begin{array}{c}
m(n-1) \\
s_{l}
\end{array}\right) \\
& +\frac{m(n-1)}{b_{1}+b_{2}+\cdots+b_{n}-1}\left(\begin{array}{c}
b_{1}+b_{2}+\cdots+b_{n}-1 \\
b_{1}-1, b_{2}, \ldots, b_{n}
\end{array}\right) \\
& \times \sum_{j=2}^{l}\left(\begin{array}{c}
m(n-1) \\
s_{2}
\end{array}\right) \cdots\left(\begin{array}{c}
m(n-1)-1 \\
s_{j}-2
\end{array}\right) \cdots\left(\begin{array}{c}
m(n-1) \\
s_{l}
\end{array}\right)
\end{aligned}
$$

if $b_{1}+2 b_{2}+\cdots+n b_{n}=n$.

Remark. The conditions in the statement of the corollary imply that

$$
s_{1}+b_{1}+b_{2}+\cdots+b_{n}=n \text {. }
$$

The reader should recall from Section 7 that non-zero blocks of elements $\pi$ of $\widetilde{N C}^{m}\left(D_{n}\right)$ occur in pairs since, with a block $B$ of $\pi, \bar{B}$ is also a block of $\pi$. The condition $b_{1}+2 b_{2}+\cdots+n b_{n}<n-1$, which is required for formula (8.33) to hold, implies that $\pi_{1}$ must contain a zero block of size $2\left(n-b_{1}-2 b_{2}-\cdots-n b_{n}\right)$, while the equality $b_{1}+2 b_{2}+\cdots+n b_{n}=n$, which is required for formula (8.34) to hold, implies that $\pi_{1}$ contains no zero block. The extra condition on zero blocks that are imposed on elements of $\widetilde{N C}^{m}\left(D_{n}\right)$ implies that $b_{1}+2 b_{2}+\cdots+n b_{n}$ cannot be equal to $n-1$.

Proof. Again, the arguments are completely analogous to those of the proof of Corollary 12. Here we need Theorem 15 with $d=1, l$ replaced by $l-1, s_{i}$ replaced by $s_{i+1}, i=1,2, \ldots, l-1, m_{1}=m_{2}=\cdots=m_{l-1}=m$, and $T_{1}$ of the type

$$
D_{n-b_{1}-2 b_{2}-\cdots-n b_{n}} * A_{1}^{b_{2}} * A_{2}^{b_{3}} * \cdots * A_{n-1}^{b_{n}}
$$

in the case that $b_{1}+2 b_{2}+\cdots+n b_{n}<n-1$, respectively

$$
A_{1}^{b_{2}} * A_{2}^{b_{3}} * \cdots * A_{n-1}^{b_{n}}
$$

if not. So, depending on the case which we are in, we have to apply (8.29), respectively (8.30). If we also take into account (8.35), then we arrive at the claimed result after little manipulation. Since we have already done similar calculations several times, the details are left to the reader.

This result is new even for $m=1$, that is, for the poset of type $D_{n}$ non-crossing partitions of Athanasiadis and Reiner [5], and of Bessis and Corran [9]. Not only does it imply all known results on these non-crossing partitions and their extension 
to $m$-divisible type $D_{n}$ non-crossing partitions due to Armstrong, it allows us as well to solve several open enumeration problems on the $m$-divisible type $D_{n}$ noncrossing partitions. We state these new results separately in the corollaries below.

To begin with, if we set $l=2$ in Corollary 16, then we obtain the following extension to $\widetilde{N C}^{m}\left(D_{n}\right)$ of Athanasiadis and Reiner's result [5. Theorem 1.3] on the number of type $D_{n}$ non-crossing partitions with a given block structure.

Corollary 17. The number of all elements of $\widetilde{N C}\left(D_{n}\right)$ which have $2 b_{i}$ non-zero blocks of size mi equals

$$
\left(\begin{array}{c}
b_{1}+b_{2}+\cdots+b_{n} \\
b_{1}, b_{2}, \ldots, b_{n}
\end{array}\right)\left(\begin{array}{c}
m(n-1) \\
b_{1}+b_{2}+\cdots+b_{n}
\end{array}\right)
$$

if $b_{1}+2 b_{2}+\cdots+n b_{n}<n-1$ and

$$
\begin{aligned}
2\left(\begin{array}{c}
b_{1}+b_{2}+\cdots+b_{n} \\
b_{1}, b_{2}, \ldots, b_{n}
\end{array}\right) & \left(\begin{array}{c}
m(n-1) \\
b_{1}+b_{2}+\cdots+b_{n}
\end{array}\right) \\
& +\left(\begin{array}{c}
b_{1}+b_{2}+\cdots+b_{n}-1 \\
b_{1}-1, b_{2}, \ldots, b_{n}
\end{array}\right)\left(\begin{array}{c}
m(n-1) \\
b_{1}+b_{2}+\cdots+b_{n}-1
\end{array}\right)
\end{aligned}
$$

if $b_{1}+2 b_{2}+\cdots+n b_{n}=n$.

On the other hand, if we sum the expression (8.33), respectively (8.34), over all $s_{2}, s_{3}, \ldots, s_{l}$ with $s_{2}+s_{3}+\cdots+s_{l}=n-s_{1}$, then we obtain the following generalisation.

Corollary 18. The number of all multi-chains $\pi_{1} \leq \pi_{2} \leq \cdots \leq \pi_{l-1}$ in $\widetilde{N C}{ }^{m}\left(D_{n}\right)$ in which $\pi_{1}$ has $2 b_{i}$ non-zero blocks of size mi equals

$$
\left(\begin{array}{c}
b_{1}+b_{2}+\cdots+b_{n} \\
b_{1}, b_{2}, \ldots, b_{n}
\end{array}\right)\left(\begin{array}{c}
(l-1) m(n-1) \\
b_{1}+b_{2}+\cdots+b_{n}
\end{array}\right)
$$

if $b_{1}+2 b_{2}+\cdots+n b_{n}<n-1$, and

$$
\begin{aligned}
2\left(\begin{array}{c}
b_{1}+b_{2}+\cdots+b_{n} \\
b_{1}, b_{2}, \ldots, b_{n}
\end{array}\right) & \left(\begin{array}{c}
(l-1) m(n-1) \\
b_{1}+b_{2}+\cdots+b_{n}
\end{array}\right) \\
+ & \left(\begin{array}{c}
b_{1}+b_{2}+\cdots+b_{n}-1 \\
b_{1}-1, b_{2}, \ldots, b_{n}
\end{array}\right)\left(\begin{array}{c}
(l-1) m(n-1) \\
b_{1}+b_{2}+\cdots+b_{n}-1
\end{array}\right)
\end{aligned}
$$

if $b_{1}+2 b_{2}+\cdots+n b_{n}=n$.

Next we sum the expressions (8.33) and (8.34) over all possible $b_{1}, b_{2}, \ldots, b_{n}$; that is, we sum (8.33) over $b_{2}+2 b_{3}+\cdots+(n-1) b_{n}<s_{1}-1$, and we sum the expression (8.34) over $b_{2}+2 b_{3}+\cdots+(n-1) b_{n}=s_{1}$. With the help of Lemma 4 
and the simple binomial summation (6.24), these sums can indeed be evaluated. In this manner, we obtain the following result on rank-selected chain enumeration in $\widetilde{N C}^{m}\left(D_{n}\right)$.

Corollary 19. The number of all multi-chains $\pi_{1} \leq \pi_{2} \leq \cdots \leq \pi_{l-1}$ in $\widetilde{N C}^{m}\left(D_{n}\right)$ $\cong N C^{m}\left(D_{n}\right)$, where $\pi_{i}$ is of rank $s_{1}+s_{2}+\cdots+s_{i}, i=1,2, \ldots, l-1$, equals

$$
\begin{aligned}
& 2\left(\begin{array}{c}
n-1 \\
s_{1}
\end{array}\right)\left(\begin{array}{c}
m(n-1) \\
s_{2}
\end{array}\right) \cdots\left(\begin{array}{c}
m(n-1) \\
s_{l}
\end{array}\right) \\
& +m \sum_{j=2}^{l}\left(\begin{array}{c}
n-1 \\
s_{1}
\end{array}\right)\left(\begin{array}{c}
m(n-1) \\
s_{2}
\end{array}\right) \cdots\left(\begin{array}{c}
m(n-1)-1 \\
s_{j}-2
\end{array}\right) \cdots\left(\begin{array}{c}
m(n-1) \\
s_{l}
\end{array}\right) \\
& +\left(\begin{array}{c}
n-2 \\
s_{1}-2
\end{array}\right)\left(\begin{array}{c}
m(n-1) \\
s_{2}
\end{array}\right) \cdots\left(\begin{array}{c}
m(n-1) \\
s_{l}
\end{array}\right) .
\end{aligned}
$$

This formula extends Athanasiadis and Reiner's formula [5, Theorem 1.2(ii)] from $N C\left(D_{n}\right)$ to $\widetilde{N C}^{m}\left(D_{n}\right)$. Setting $l=2$, we obtain a formula for the number of all elements in $\widetilde{N C}^{m}\left(D_{n}\right) \cong N C^{m}\left(D_{n}\right)$ with a given number of blocks (equivalently, of given rank); cf. [1, Theorem 4.6.3]. Next, summing (8.40) over all non-negative integers $s_{1}, s_{2}, \ldots, s_{l}$ with $s_{1}+s_{2}+\cdots+s_{l}=n$ by means of the multivariate $\mathrm{Chu}^{-}$ Vandermonde summation, we find that the total number of multi-chains $\pi_{1} \leq \pi_{2} \leq$ $\cdots \leq \pi_{l-1}$ in $\widetilde{N C}^{m}\left(D_{n}\right) \cong N C^{m}\left(D_{n}\right)$ is given by

$$
\begin{aligned}
2\left(\begin{array}{c}
((l-1) m+1)(n-1) \\
n
\end{array}\right)+\left(\begin{array}{c}
((l-1) m+1)(n-1) \\
n-1
\end{array}\right) & \\
= & \frac{2(l-1) m(n-1)+n}{n}\left(\begin{array}{c}
(l-1) m+1)(n-1) \\
n-1
\end{array}\right)
\end{aligned}
$$

thus recovering the formula [1, Theorem 3.6.9] for the zeta polynomial of the poset of generalised non-crossing partitions for type $D_{n}$. The special case $l=2$ of (8.41) gives the well-known fact that the cardinality of $\widetilde{N C^{m}}\left(D_{n}\right) \cong N C^{m}\left(D_{n}\right)$ is $\frac{2 m(n-1)+n}{n}\left(\begin{array}{c}(m+1)(n-1) \\ n-1\end{array}\right)$ (cf. [1, Theorem 3.5.3]).

In the following section, Corollary [19 will enable us to provide a new proof of Armstrong's $F=M($ Ex- $)$ Conjecture in type $D_{n}$.

\section{Proof of the $F=M$ Conjecture for type $D$}

Armstrong's $F=M$ (Ex-)Conjecture [1, Conjecture 5.3.2], which extends an earlier conjecture of Chapoton [17, relates the " $F$-triangle" of the generalised cluster complex of Fomin and Reading [19] to the " $M$-triangle" of Armstrong's generalised non-crossing partitions. The $F$-triangle is a certain refined face count in the generalised cluster complex. We do not give the definition here and, instead, refer the reader to [1, 27, because it will not be important in what follows. It suffices to know that, again fixing a finite root system $\Phi$ of rank $n$ and a positive integer $m$, the $F$-triangle $F_{\Phi}^{m}(x, y)$ for the generalised cluster complex $\Delta^{m}(\Phi)$ is a polynomial in $x$ and $y$ and that it was computed in [27] for all types. What we need here is 
that it was shown in [27. Sec. 11, Prop. D] that

$$
\begin{aligned}
& (1-x y)^{n} F_{D_{n}}^{m}\left(\frac{x(1+y)}{1-x y}, \frac{x y}{1-x y}\right) \\
& =\sum_{r, s \geq 0} x^{s} y^{r}\left(2\left(\begin{array}{c}
n-1 \\
s-1
\end{array}\right)\left(\begin{array}{c}
m(n-1) \\
r
\end{array}\right)\left(\begin{array}{c}
m(n-1)+s-r-1 \\
s-r
\end{array}\right)\right. \\
& +\left(\begin{array}{c}
n-2 \\
s
\end{array}\right)\left(\begin{array}{c}
m(n-1) \\
r
\end{array}\right)\left(\begin{array}{c}
m(n-1)+s-r-1 \\
s-r
\end{array}\right) \\
& +m\left(\begin{array}{c}
n-1 \\
s-1
\end{array}\right)\left(\begin{array}{c}
m(n-1)-1 \\
r-2
\end{array}\right)\left(\begin{array}{c}
m(n-1)+s-r-1 \\
s-r
\end{array}\right) \\
& \left.-m\left(\begin{array}{c}
n-1 \\
s-1
\end{array}\right)\left(\begin{array}{c}
m(n-1) \\
r
\end{array}\right)\left(\begin{array}{c}
m(n-1)+s-r-2 \\
s-r-2
\end{array}\right)\right) .
\end{aligned}
$$

The " $M$-triangle" of $N C^{m}(\Phi)$ is the polynomial defined by

$$
M_{\Phi}^{m}(x, y)=\sum_{u, w \in N C^{m}(\Phi)} \mu(u, w) x^{\mathrm{rk} u} y^{\mathrm{rk} w}
$$

where $\mu(u, w)$ is the Möbius function in $N C^{m}(\Phi)$. It is called a "triangle" because the Möbius function $\mu(u, w)$ vanishes unless $u \leq w$, and, thus, the only coefficients in the polynomial which may be non-zero are the coefficients of $x^{k} y^{l}$ with $0 \leq k \leq$ $l \leq n$.

An equivalent object is the dual $M$-triangle, which is defined by

$$
\left(M_{\Phi}^{m}\right)^{*}(x, y)=\sum_{u, w \in\left(N C^{m}(\Phi)\right)^{*}} \mu^{*}(u, w) x^{\mathrm{rk}^{*} w} y^{\mathrm{rk}^{*} u}
$$

where $\left(N C^{m}(\Phi)\right)^{*}$ denotes the poset dual to $N C^{m}(\Phi)$ (i.e., the poset which arises from $N C^{m}(\Phi)$ by reversing all order relations), where $\mu^{*}$ denotes the Möbius function in $\left(N C^{m}(\Phi)\right)^{*}$ and where $\mathrm{rk}^{*}$ denotes the rank function in $\left(N C^{m}(\Phi)\right)^{*}$. It is equivalent since, obviously, we have

$$
\left(M_{\Phi}^{m}\right)^{*}(x, y)=(x y)^{n} M_{\Phi}^{m}(1 / x, 1 / y) .
$$

Given this notation, Armstrong's $F=M$ (Ex-)Conjecture [1, Conjecture 5.3.2] reads as follows.

Conjecture FM. For any finite root system $\Phi$ of rank $n$, we have

$$
F_{\Phi}^{m}(x, y)=y^{n} M_{\Phi}^{m}\left(\frac{1+y}{y-x}, \frac{y-x}{y}\right) .
$$

Equivalently,

$$
(1-x y)^{n} F_{\Phi}^{m}\left(\frac{x(1+y)}{1-x y}, \frac{x y}{1-x y}\right)=\sum_{u, w \in\left(N C^{m}(\Phi)\right)^{*}} \mu^{*}(u, w)(-x)^{\mathrm{rk}^{*} w}(-y)^{\mathrm{rk}^{*} u}
$$

So, equation (9.1) provides an expression for the left-hand side of (9.3) for $\Phi=$ $D_{n}$. With our result on rank-selected chain enumeration in $N C^{m}\left(D_{n}\right)$ given in Corollary 19, we are now able to calculate the right-hand side of (9.3) directly. As we mentioned already in the Introduction, together with the results from [27, 28], 
this completes a computational case-by-case proof of Conjecture FM. A case-free proof had been found earlier by Tzanaki in [38].

The only ingredient that we need for the proof is the well-known link between chain enumeration and the Möbius function. (The reader should consult [33, Sec. 3.11] for more information on this topic.) Given a poset $P$ and two elements $u$ and $w, u \leq w$, in the poset, the zeta polynomial of the interval $[u, w]$, denoted by $Z(u, w ; z)$, is the number of (multi-)chains from $u$ to $w$ of length $z$. (It can be shown that this is indeed a polynomial in $z$.) Then the Möbius function of $u$ and $w$ is equal to $\mu(u, w)=Z(u, w ;-1)$.

Proof of Conjecture FM in type $D_{n}$. We now compute the right-hand side of (9.3), that is,

$$
\sum_{u, w \in\left(N C^{m}\left(D_{n}\right)\right)^{*}} \mu^{*}(u, w)(-x)^{\mathrm{rk}^{*} w}(-y)^{\mathrm{rk}^{*} u} .
$$

In order to compute the coefficient of $x^{s} y^{r}$ in this expression,

$$
(-1)^{r+s} \sum_{\begin{array}{c}
u, w \in\left(N C^{m}\left(D_{n}\right)\right)^{*} \\
\text { with } \mathrm{rk}^{*} u=r \text { and } \mathrm{rk}^{*} w=s
\end{array}} \mu^{*}(u, w),
$$

we compute the sum of all corresponding zeta polynomials (in the variable $z$ ), multiplied by $(-1)^{r+s}$,

$$
(-1)^{r+s} \sum_{\begin{array}{c}
u, w \in\left(N C^{m}\left(D_{n}\right)\right)^{*} \\
\text { with } \operatorname{rk}^{*} u=r \text { and } \operatorname{rk}^{*} w=s
\end{array}} Z(u, w ; z),
$$

and then put $z=-1$.

For computing this sum of zeta polynomials, we must set $l=z+2, n-s_{1}=s$, $s_{l}=r, s_{2}+s_{3}+\cdots+s_{l-1}=s-r$ in (8.40), and then sum the resulting expression over all possible $s_{2}, s_{3}, \ldots, s_{l-1}$. (The reader should keep in mind that the roles of $s_{1}, s_{2}, \ldots, s_{l}$ in Corollary 19 have to be reversed, since we are aiming at computing zeta polynomials in the poset dual to $N C^{m}\left(D_{n}\right)$.) By using the Chu-Vandermonde summation, one obtains

$$
\begin{aligned}
& 2\left(\begin{array}{c}
m(n-1) \\
r
\end{array}\right)\left(\begin{array}{c}
z m(n-1) \\
s-r
\end{array}\right)\left(\begin{array}{c}
n-1 \\
s-1
\end{array}\right)+m\left(\begin{array}{c}
m(n-1)-1 \\
r-2
\end{array}\right)\left(\begin{array}{c}
z m(n-1) \\
s-r
\end{array}\right)\left(\begin{array}{c}
n-1 \\
s-1
\end{array}\right) \\
+ & z m\left(\begin{array}{c}
m(n-1) \\
r
\end{array}\right)\left(\begin{array}{c}
z m(n-1)-1 \\
s-r-2
\end{array}\right)\left(\begin{array}{c}
n-1 \\
s-1
\end{array}\right)+\left(\begin{array}{c}
m(n-1) \\
r
\end{array}\right)\left(\begin{array}{c}
z m(n-1) \\
s-r
\end{array}\right)\left(\begin{array}{c}
n-2 \\
s
\end{array}\right) .
\end{aligned}
$$

If we put $z=-1$ in this expression and multiply it by $(-1)^{r+s}$, then we obtain exactly the coefficient of $x^{s} y^{r}$ in (9.1).

\section{A conjecture of Armstrong on maximal intervals CONTAINING A RANDOM MULTICHAIN}

Given a finite root system of rank $n$, Conjecture 3.5.13 in [1] says the following: If we choose an l-multichain uniformly at random from the set

$$
\left\{\pi_{1} \leq \pi_{2} \leq \cdots \leq \pi_{l}: \pi_{i} \in N C^{m}(\Phi), i=1, \ldots, l \text {, and } \operatorname{rk}\left(\pi_{1}\right)=i\right\},
$$


then the expected number of maximal intervals in $N C^{m}(\Phi)$ containing this multichain is

$$
\frac{\operatorname{Nar}^{m}(\Phi, n-i)}{\operatorname{Nar}^{1}(\Phi, n-i)}
$$

where $\operatorname{Nar}^{m}(\Phi, i)$ is the $i$-th $F u ß-N a r a y a n a$ number associated to $N C^{m}(\Phi)$, that is, the number of elements of $N C^{m}(\Phi)$ of rank $i$. In particular, this expected value is independent of $l$.

We show in this section that, for types $A_{n}$ and $B_{n}$, the conjecture follows easily from Edelman's (8.14), respectively Armstrong's (8.25) (presumably, this fact constituted the evidence for setting up the conjecture), while an analogous computation using our new result (8.40) demonstrates that it fails for type $D_{n}$. At the end of this section, we comment on what we think happens for the exceptional types.

The computation of the expected value in the above conjecture can be approached in the following way. One first observes that a maximal interval in $N C^{m}(\Phi)$ is an interval between an element $\pi_{0}$ of rank 0 and the global maximum $(c ; \varepsilon, \ldots, \varepsilon)$. Therefore, to compute the proposed expected value, we may count the number of chains

$$
\pi_{0} \leq \pi_{1} \leq \pi_{2} \leq \cdots \leq \pi_{l}, \quad \operatorname{rk}\left(\pi_{0}\right)=0 \text { and } \operatorname{rk}\left(\pi_{1}\right)=i,
$$

and divide this number by the total number of all chains in 10.1). Clearly, in types $A_{n}, B_{n}$, and $D_{n}$, this kind of chain enumeration can be easily accessed by (8.14), (8.25), and (8.40), respectively.

We begin with type $A_{n}$. By (8.14), the number of chains (10.3) equals

$$
\begin{aligned}
& \sum_{s_{2}+\cdots+s_{l+1}=n-i} \frac{1}{n+1}\left(\begin{array}{c}
n+1 \\
0
\end{array}\right)\left(\begin{array}{c}
m(n+1) \\
i
\end{array}\right)\left(\begin{array}{c}
m(n+1) \\
s_{2}
\end{array}\right) \cdots\left(\begin{array}{c}
m(n+1) \\
s_{l+1}
\end{array}\right) \\
& =\frac{1}{n+1}\left(\begin{array}{c}
m(n+1) \\
i
\end{array}\right)\left(\begin{array}{c}
m l(n+1) \\
n-i
\end{array}\right),
\end{aligned}
$$

while the number of chains in (10.1) equals

$$
\begin{aligned}
\sum_{s_{2}+\cdots+s_{l+1}=n-i} \frac{1}{n+1}\left(\begin{array}{c}
n+1 \\
i
\end{array}\right)\left(\begin{array}{c}
m(n+1) \\
s_{2}
\end{array}\right) \cdots\left(\begin{array}{c}
m(n+1) \\
s_{l+1}
\end{array}\right) \\
=\frac{1}{n+1}\left(\begin{array}{c}
n+1 \\
i
\end{array}\right)\left(\begin{array}{c}
m l(n+1) \\
n-i
\end{array}\right) .
\end{aligned}
$$

In both cases, we used the multivariate $\mathrm{Chu}$-Vandermonde summation to evaluate the sums over $s_{2}, \ldots, s_{l+1}$. The quotient of the two numbers is

$$
\frac{\frac{1}{n+1}\left(\begin{array}{c}
m(n+1) \\
i
\end{array}\right)}{\frac{1}{n+1}\left(\begin{array}{c}
n+1 \\
i
\end{array}\right)}=\frac{\frac{1}{n+1}\left(\begin{array}{c}
n+1 \\
n-i
\end{array}\right)\left(\begin{array}{c}
m(n+1) \\
i
\end{array}\right)}{\frac{1}{n+1}\left(\begin{array}{c}
n+1 \\
n-i
\end{array}\right)\left(\begin{array}{c}
n+1 \\
i
\end{array}\right)}
$$

which by (8.14) with $n$ replaced by $n+1, l=2, s_{1}=n-i$, and $s_{2}=i$ agrees indeed with (10.2) for $\Phi=A_{n}$. 
For type $B_{n}$, there is an analogous computation using (8.25), the details of which we leave to the reader. The result is that the desired expected value equals

$$
\frac{\left(\begin{array}{c}
m n \\
i
\end{array}\right)}{\left(\begin{array}{c}
n \\
i
\end{array}\right)}=\frac{\left(\begin{array}{c}
n \\
n-i
\end{array}\right)\left(\begin{array}{c}
m n \\
i
\end{array}\right)}{\left(\begin{array}{c}
n \\
n-i
\end{array}\right)\left(\begin{array}{c}
n \\
i
\end{array}\right)}
$$

which by (8.25) with $l=2, s_{1}=n-i$, and $s_{2}=i$ agrees indeed with (10.2) for $\Phi=B_{n}$.

The analogous computation for type $D_{n}$ uses (8.40). The number of chains (10.3) equals

$$
\begin{aligned}
& 2 \sum_{s_{2}+\cdots+s_{l+1}=n-i}\left(\begin{array}{c}
n-1 \\
0
\end{array}\right)\left(\begin{array}{c}
m(n-1) \\
i
\end{array}\right)\left(\begin{array}{c}
m(n-1) \\
s_{2}
\end{array}\right) \cdots\left(\begin{array}{c}
m(n-1) \\
s_{l+1}
\end{array}\right) \\
& +m \sum_{s_{2}+\cdots+s_{l+1}=n-i}\left(\begin{array}{c}
n-1 \\
0
\end{array}\right)\left(\begin{array}{c}
m(n-1)-1 \\
i-2
\end{array}\right)\left(\begin{array}{c}
m(n-1) \\
s_{2}
\end{array}\right) \cdots\left(\begin{array}{c}
m(n-1) \\
s_{l+1}
\end{array}\right) \\
& \quad+m \sum_{j=3}^{l+1} \sum_{s_{2}+\cdots+s_{l+1}=n-i}\left(\begin{array}{c}
n-1 \\
0
\end{array}\right)\left(\begin{array}{c}
m(n-1) \\
i
\end{array}\right)\left(\begin{array}{c}
m(n-1) \\
s_{2}
\end{array}\right) \\
& =2\left(\begin{array}{c}
m(n-1) \\
i
\end{array}\right)\left(\begin{array}{c}
m(n-1)-1 \\
s_{j}-2
\end{array}\right) \cdots\left(\begin{array}{c}
m(n-1) \\
s_{l+1}
\end{array}\right)
\end{aligned}
$$

$$
+m(l-1)\left(\begin{array}{c}
m(n-1) \\
i
\end{array}\right)\left(\begin{array}{c}
m l(n-1)-1 \\
n-i-2
\end{array}\right),
$$

while the number of chains in (10.1) equals

$$
\begin{gathered}
2 \sum_{s_{2}+\cdots+s_{l+1}=n-i}\left(\begin{array}{c}
n-1 \\
i
\end{array}\right)\left(\begin{array}{c}
m(n-1) \\
s_{2}
\end{array}\right) \cdots\left(\begin{array}{c}
m(n-1) \\
s_{l+1}
\end{array}\right) \\
+m \sum_{j=2}^{l+1} \sum_{s_{2}+\cdots+s_{l+1}=n-i}\left(\begin{array}{c}
n-1 \\
i
\end{array}\right)\left(\begin{array}{c}
m(n-1) \\
s_{2}
\end{array}\right) \\
\ldots\left(\begin{array}{c}
m(n-1)-1 \\
s_{j}-2
\end{array}\right) \cdots\left(\begin{array}{c}
m(n-1) \\
s_{l+1}
\end{array}\right) \\
+\quad \sum_{s_{2}+\cdots+s_{l+1}=n-i}\left(\begin{array}{c}
n-2 \\
i-2
\end{array}\right)\left(\begin{array}{c}
m(n-1) \\
s_{2}
\end{array}\right) \cdots\left(\begin{array}{c}
m(n-1) \\
s_{l+1}
\end{array}\right) \\
=2\left(\begin{array}{c}
n-1 \\
i
\end{array}\right)\left(\begin{array}{c}
m l(n-1) \\
n-i
\end{array}\right)+m l\left(\begin{array}{c}
n-1 \\
i
\end{array}\right)\left(\begin{array}{c}
m l(n-1)-1 \\
n-i-2
\end{array}\right) \\
+\left(\begin{array}{c}
n-2 \\
i-2
\end{array}\right)\left(\begin{array}{c}
m l(n-1) \\
n-i
\end{array}\right) .
\end{gathered}
$$

The quotient of (10.4) and (10.5) gives the desired expected value. It is, however, not independent of $l$, and therefore Armstrong's conjecture does not hold for $\Phi=$ $D_{n}$. 
In the case that $\Phi$ is of exceptional type, then, as we outline in the next section, the knowledge of the corresponding decomposition numbers (see the appendix) allows one to access the rank selected chain enumeration. Using this, the approach for computing the expected value proposed by Armstrong that we used above for the classical types can be carried through as well for the exceptional types. We have not done this, but we expect that, similarly to the case of $D_{n}$, for most exceptional types the expected value will depend on $l$, so that Armstrong's conjecture will probably also fail in these cases.

\section{Chain enumeration in the poset of Generalised NON-CROssing PARTITIONS FOR THE EXCEPTIONAL TYPES}

Although it is not the main topic of our paper, we want to briefly demonstrate in this section that the knowledge of the decomposition numbers also enables one to do refined enumeration in the generalised non-crossing partition posets $N C^{m}(\Phi)$ for exceptional root systems $\Phi$ (of rank $n$ ). We restrict the following considerations to the rank-selected chain enumeration. This means that we want to count the number of all multi-chains $\pi_{1} \leq \pi_{2} \leq \cdots \leq \pi_{l-1}$ in $N C^{m}(\Phi)$, where $\pi_{i}$ is of rank $s_{1}+s_{2}+\cdots+s_{i}, i=1,2, \ldots, l-1$. Let us denote this number by $R_{\Phi}\left(s_{1}, s_{2}, \ldots, s_{l}\right)$, with $s_{l}=n-s_{1}-s_{2}-\cdots-s_{l}$. Now, the considerations at the beginning of the proof of Corollary 12 leading to the factorisation (8.12) with rank constraints on the factors, are also valid for $N C^{m}(\Phi)$ instead of $N C^{m}\left(A_{n-1}\right)$, that is, they are independent of the underlying root system. Hence, to determine the number $R_{\Phi}\left(s_{1}, s_{2}, \ldots, s_{l}\right)$, we have to count all possible factorisations

$$
c=w_{0}^{(1)}\left(u_{1}^{(2)} u_{1}^{(3)} \cdots u_{1}^{(l)}\right)\left(u_{2}^{(2)} u_{2}^{(3)} \cdots u_{2}^{(l)}\right) \cdots\left(u_{m}^{(2)} u_{m}^{(3)} \cdots u_{m}^{(l)}\right)
$$

under the rank constraints (8.11) and $\ell_{T}\left(w_{0}^{(1)}\right)=s_{1}$, where $c$ is a Coxeter element in $W(\Phi)$. As we remarked in the proof of Corollary 12, equivalently we may count all factorisations

$$
c=w_{0}^{(1)}\left(u_{1}^{(2)} u_{2}^{(2)} \cdots u_{m}^{(2)}\right)\left(u_{1}^{(3)} u_{2}^{(3)} \cdots u_{m}^{(3)}\right) \cdots\left(u_{1}^{(l)} u_{2}^{(l)} \cdots u_{m}^{(l)}\right)
$$

which satisfy (8.11) and $\ell_{T}\left(w_{0}^{(1)}\right)=s_{1}$. We can now obtain an explicit expression by fixing first the types of $w_{0}^{(1)}$ and all the $u_{i}^{(j)}$ 's. Under these constraints, the number of factorisations (11.1) is just the corresponding decomposition number. Subsequently, we sum the resulting expressions over all possible types.

Before we are able to state the formula which we obtain in this way, we need to recall some standard integer partition notation (cf. e.g. 34, Sec. 7.2]). An integer partition $\lambda$ (with $n$ parts) is an $n$-tuple $\lambda=\left(\lambda_{1}, \lambda_{2}, \ldots, \lambda_{n}\right)$ of integers satisfying $\lambda_{1} \geq \lambda_{2} \geq \cdots \geq \lambda_{n} \geq 0$. It is called an integer partition of $N$, written in symbolic notation as $\lambda \vdash N$, if $\lambda_{1}+\lambda_{2}+\cdots+\lambda_{n}=N$. The number of parts (components) of $\lambda$ of size $i$ is denoted by $m_{i}(\lambda)$.

Then, again making use of the notation for the multinomial coefficient introduced in Lemma 4, the expression for $R_{\Phi}\left(s_{1}, s_{2}, \ldots, s_{l}\right)$ which we obtain in the way described above is

$$
\sum^{\prime} N_{\Phi}\left(T_{0}^{(1)}, T_{1}^{(2)}, T_{2}^{(2)}, \ldots, T_{n}^{(l)}\right) \prod_{j=2}^{l}\left(\begin{array}{c}
m \\
m_{1}\left(\lambda^{(j)}\right), m_{2}\left(\lambda^{(j)}\right), \ldots, m_{n}\left(\lambda^{(j)}\right)
\end{array}\right)
$$


where $\sum^{\prime}$ is taken over all integer partitions $\lambda^{(2)}, \lambda^{(3)}, \ldots, \lambda^{(l)}$ satisfying $\lambda^{(2)} \vdash s_{2}$, $\lambda^{(3)} \vdash s_{3}, \ldots, \lambda^{(l)} \vdash s_{l}$, over all types $T_{0}^{(1)}$ with $\operatorname{rk}\left(T_{0}^{(1)}\right)=s_{1}$, and over all types $T_{i}^{(j)}$ with $\operatorname{rk}\left(T_{i}^{(j)}\right)=\lambda_{i}^{(j)}, i=1,2, \ldots, n, j=2,3, \ldots, l$.

By way of example, using this formula and the values of the decomposition numbers $N_{E_{8}}(\ldots)$ given in Appendix A.7 (and a computer), we obtain that the number $R_{E_{8}}(4,2,1,1)$ of all chains $\pi_{1} \leq \pi_{2} \leq \pi_{3}$ in $N C^{m}\left(E_{8}\right)$, where $\pi_{1}$ is of rank $4, \pi_{2}$ is of rank 6 , and $\pi_{3}$ is of rank 7 , is given by

$$
\frac{75 m^{3}(8055 m-1141)}{2}
$$

(which, by the independence (2.2) of decomposition numbers from the order of the types, is also equal to $R_{E_{8}}(4,1,2,1)$ and $R_{E_{8}}(4,1,1,2)$ ), while the number $R_{E_{8}}(2,4,1,1)$ of all chains $\pi_{1} \leq \pi_{2} \leq \pi_{3}$ in $N C^{m}\left(E_{8}\right)$, where $\pi_{1}$ is of rank $2, \pi_{2}$ is of rank 6 , and $\pi_{3}$ is of rank 7 , is given by

$$
\frac{75 m^{3}\left(73125 m^{3}-58950 m^{2}+15635 m-2154\right)}{8}
$$

(which is also equal to $R_{E_{8}}(2,1,4,1)$ and $R_{E_{8}}(2,1,1,4)$ ).

\section{Appendix A. The Decomposition numbers for the EXCEPtional types}

A.1. The decomposition numbers for type $I_{2}(a)$ [27, Sec. 13]. We have $N_{I_{2}(a)}\left(I_{2}(a)\right)=1, N_{I_{2}(a)}\left(A_{1}, A_{1}\right)=a, N_{I_{2}(a)}\left(A_{1}\right)=a, N_{I_{2}(a)}(\emptyset)=1$, with all other numbers $N_{I_{2}(a)}\left(T_{1}, T_{2}, \ldots, T_{d}\right)$ being zero.

A.2. The decomposition numbers for type $H_{3}$ [27, Sec. 14]. We have $N_{H_{3}}\left(H_{3}\right)=1, N_{H_{3}}\left(A_{1}^{2}, A_{1}\right)=5, N_{H_{3}}\left(A_{2}, A_{1}\right)=5, N_{H_{3}}\left(I_{2}(5), A_{1}\right)=5$, $N_{H_{3}}\left(A_{1}, A_{1}, A_{1}\right)=50$, plus the assignments implied by (2.2) and (2.3), with all other numbers $N_{H_{3}}\left(T_{1}, T_{2}, \ldots, T_{d}\right)$ being zero.

A.3. The decomposition numbers for type $H_{4}$ [27, Sec. 15]. We have $N_{H_{4}}\left(H_{4}\right)=1, N_{H_{4}}\left(A_{1} * A_{2}, A_{1}\right)=15, N_{H_{4}}\left(A_{3}, A_{1}\right)=15, N_{H_{4}}\left(H_{3}, A_{1}\right)=15$, $N_{H_{4}}\left(A_{1} * I_{2}(5), A_{1}\right)=15, N_{H_{4}}\left(A_{1}^{2}, A_{1}^{2}\right)=30, N_{H_{4}}\left(A_{1}^{2}, A_{2}\right)=30, N_{H_{4}}\left(A_{1}^{2}, I_{2}(5)\right)$ $=15, N_{H_{4}}\left(A_{2}, A_{2}\right)=5, N_{H_{4}}\left(A_{2}, I_{2}(5)\right)=15, \quad N_{H_{4}}\left(I_{2}(5), I_{2}(5)\right)=3$, $N_{H_{4}}\left(A_{1}^{2}, A_{1}, A_{1}\right)=225, \quad N_{H_{4}}\left(A_{2}, A_{1}, A_{1}\right)=150, N_{H_{4}}\left(I_{2}(5), A_{1}, A_{1}\right)=90$, $N_{H_{4}}\left(A_{1}, A_{1}, A_{1}, A_{1}\right)=1350$, plus the assignments implied by (2.2) and (2.3), with all other numbers $N_{H_{4}}\left(T_{1}, T_{2}, \ldots, T_{d}\right)$ being zero.

A.4. The decomposition numbers for type $F_{4}\left[27\right.$, Sec. 16]. We have $N_{F_{4}}\left(F_{4}\right)$ $=1, N_{F_{4}}\left(A_{1} * A_{2}, A_{1}\right)=12, N_{F_{4}}\left(B_{3}, A_{1}\right)=12, N_{F_{4}}\left(A_{1}^{2}, A_{1}^{2}\right)=12, N_{F_{4}}\left(A_{1}^{2}, B_{2}\right)=$ $12, N_{F_{4}}\left(A_{2}, A_{2}\right)=16, N_{F_{4}}\left(B_{2}, B_{2}\right)=3, N_{F_{4}}\left(A_{1}^{2}, A_{1}, A_{1}\right)=72, N_{F_{4}}\left(A_{2}, A_{1}, A_{1}\right)=$ $48, N_{F_{4}}\left(B_{2}, A_{1}, A_{1}\right)=36, N_{F_{4}}\left(A_{1}, A_{1}, A_{1}, A_{1}\right)=432$, plus the assignments implied by (2.2) and (2.3), with all other numbers $N_{F_{4}}\left(T_{1}, T_{2}, \ldots, T_{d}\right)$ being zero.

A.5. The decomposition numbers for type $E_{6}\left[27\right.$, Sec.17]. We have $N_{E_{6}}\left(E_{6}\right)$ $=1, N_{E_{6}}\left(A_{1} * A_{2}^{2}, A_{1}\right)=6, N_{E_{6}}\left(A_{1} * A_{4}, A_{1}\right)=12, N_{E_{6}}\left(A_{5}, A_{1}\right)=6, N_{E_{6}}\left(D_{5}, A_{1}\right)$

$=12, N_{E_{6}}\left(A_{1}^{2} * A_{2}, A_{2}\right)=36, N_{E_{6}}\left(A_{2}^{2}, A_{2}\right)=8, N_{E_{6}}\left(A_{1} * A_{3}, A_{2}\right)=24, N_{E_{6}}\left(A_{4}, A_{2}\right)$ $=24, N_{E_{6}}\left(D_{4}, A_{2}\right)=4, N_{E_{6}}\left(A_{1}^{2} * A_{2}, A_{1}^{2}\right)=18, N_{E_{6}}\left(A_{1} * A_{3}, A_{1}^{2}\right)=36, N_{E_{6}}\left(A_{4}, A_{1}^{2}\right)$ $=36, \quad N_{E_{6}}\left(D_{4}, A_{1}^{2}\right)=18, \quad N_{E_{6}}\left(A_{1}^{3}, A_{1}^{3}\right)=12, \quad N_{E_{6}}\left(A_{1} * A_{2}, A_{1}^{3}\right)=24$, $N_{E_{6}}\left(A_{1} * A_{2}, A_{1} * A_{2}\right)=48, N_{E_{6}}\left(A_{3}, A_{1}^{3}\right)=36, N_{E_{6}}\left(A_{3}, A_{1} * A_{2}\right)=72$, $N_{E_{6}}\left(A_{3}, A_{3}\right)=27, \quad N_{E_{6}}\left(A_{1}^{2} * A_{2}, A_{1}, A_{1}\right)=144, \quad N_{E_{6}}\left(A_{2}^{2}, A_{1}, A_{1}\right)=24$, $N_{E_{6}}\left(A_{1} * A_{3}, A_{1}, A_{1}\right)=144, N_{E_{6}}\left(A_{4}, A_{1}, A_{1}\right)=144, N_{E_{6}}\left(D_{4}, A_{1}, A_{1}\right)=48$, 
$N_{E_{6}}\left(A_{1}^{3}, A_{1}^{2}, A_{1}\right)=180, N_{E_{6}}\left(A_{1}^{3}, A_{2}, A_{1}\right)=168, N_{E_{6}}\left(A_{1} * A_{2}, A_{1}^{2}, A_{1}\right)=360$, $N_{E_{6}}\left(A_{1} * A_{2}, A_{2}, A_{1}\right)=336, N_{E_{6}}\left(A_{3}, A_{1}^{2}, A_{1}\right)=378, N_{E_{6}}\left(A_{3}, A_{2}, A_{1}\right)=180$, $N_{E_{6}}\left(A_{1}^{2}, A_{1}^{2}, A_{1}^{2}\right)=432, \quad N_{E_{6}}\left(A_{2}, A_{1}^{2}, A_{1}^{2}\right)=504, \quad N_{E_{6}}\left(A_{2}, A_{2}, A_{1}^{2}\right)=288$, $N_{E_{6}}\left(A_{2}, A_{2}, A_{2}\right)=160, N_{E_{6}}\left(A_{1}^{2}, A_{1}^{2}, A_{1}, A_{1}\right)=2376, N_{E_{6}}\left(A_{2}, A_{1}^{2}, A_{1}, A_{1}\right)=1872$, $N_{E_{6}}\left(A_{2}, A_{2}, A_{1}, A_{1}\right)=1056, N_{E_{6}}\left(A_{1}^{3}, A_{1}, A_{1}, A_{1}\right)=864, N_{E_{6}}\left(A_{1} * A_{2}, A_{1}, A_{1}, A_{1}\right)$ $=1728, \quad N_{E_{6}}\left(A_{3}, A_{1}, A_{1}, A_{1}\right)=1296, \quad N_{E_{6}}\left(A_{1}^{2}, A_{1}, A_{1}, A_{1}, A_{1}\right)=10368$, $N_{E_{6}}\left(A_{2}, A_{1}, A_{1}, A_{1}, A_{1}\right)=6912, N_{E_{6}}\left(A_{1}, A_{1}, A_{1}, A_{1}, A_{1}, A_{1}\right)=41472$, plus the assignments implied by (2.2) and (2.3), with all other numbers $N_{E_{6}}\left(T_{1}, T_{2}, \ldots, T_{d}\right)$ being zero.

A.6. The decomposition numbers for type $E_{7}\left[\mathbf{2 8}\right.$, Sec. 6]. We have $N_{E_{7}}\left(E_{7}\right)$ $=1, N_{E_{7}}\left(E_{6}, A_{1}\right)=9, N_{E_{7}}\left(D_{6}, A_{1}\right)=9, N_{E_{7}}\left(A_{6}, A_{1}\right)=9, N_{E_{7}}\left(A_{1} * D_{5}, A_{1}\right)$ $=9, N_{E_{7}}\left(A_{1} * A_{5}, A_{1}\right)=9, N_{E_{7}}\left(A_{2} * D_{4}, A_{1}\right)=0, N_{E_{7}}\left(A_{2} * A_{4}, A_{1}\right)=9$, $N_{E_{7}}\left(A_{1}^{2} * D_{4}, A_{1}\right)=0, \quad N_{E_{7}}\left(A_{1}^{2} * A_{4}, A_{1}\right)=0, \quad N_{E_{7}}\left(A_{3}^{2}, A_{1}\right)=0$, $N_{E_{7}}\left(A_{1} * A_{2} * A_{3}, A_{1}\right)=9, \quad N_{E_{7}}\left(A_{1}^{3} * A_{3}, A_{1}\right)=0, N_{E_{7}}\left(A_{2}^{3}, A_{1}\right)=0$, $N_{E_{7}}\left(A_{1}^{2} * A_{2}^{2}, A_{1}\right)=0, N_{E_{7}}\left(A_{1}^{4} * A_{2}, A_{1}\right)=0, N_{E_{7}}\left(A_{1}^{6}, A_{1}\right)=0, N_{E_{7}}\left(D_{5}, A_{2}\right)=18$, $N_{E_{7}}\left(A_{5}, A_{2}\right)=30, \quad N_{E_{7}}\left(A_{1} * A_{4}, A_{2}\right)=54, \quad N_{E_{7}}\left(A_{1} * D_{4}, A_{2}\right)=9$, $N_{E_{7}}\left(A_{2} * A_{3}, A_{2}\right)=36, N_{E_{7}}\left(A_{1}^{2} * A_{3}, A_{2}\right)=36, N_{E_{7}}\left(A_{1} * A_{2}^{2}, A_{2}\right)=36$, $N_{E_{7}}\left(A_{1}^{3} * A_{2}, A_{2}\right)=12, N_{E_{7}}\left(A_{1}^{5}, A_{2}\right)=0, N_{E_{7}}\left(D_{5}, A_{1}^{2}\right)=54, N_{E_{7}}\left(A_{5}, A_{1}^{2}\right)=63$, $N_{E_{7}}\left(A_{1} * D_{4}, A_{1}^{2}\right)=27, N_{E_{7}}\left(A_{1} * A_{4}, A_{1}^{2}\right)=81, \quad N_{E_{7}}\left(A_{2} * A_{3}, A_{1}^{2}\right)=27$, $N_{E_{7}}\left(A_{1}^{2} * A_{3}, A_{1}^{2}\right)=27, N_{E_{7}}\left(A_{1} * A_{2}^{2}, A_{1}^{2}\right)=27, N_{E_{7}}\left(A_{1}^{3} * A_{2}, A_{1}^{2}\right)=9, N_{E_{7}}\left(A_{1}^{5}, A_{1}^{2}\right)$ $=0, N_{E_{7}}\left(D_{5}, A_{1}, A_{1}\right)=162, N_{E_{7}}\left(A_{5}, A_{1}, A_{1}\right)=216, \quad N_{E_{7}}\left(A_{1} * D_{4}, A_{1}, A_{1}\right)$ $=81, \quad N_{E_{7}}\left(A_{1} * A_{4}, A_{1}, A_{1}\right)=324, \quad N_{E_{7}}\left(A_{2} * A_{3}, A_{1}, A_{1}\right)=162$, $N_{E_{7}}\left(A_{1}^{2} * A_{3}, A_{1}, A_{1}\right)=162, N_{E_{7}}\left(A_{1} * A_{2}^{2}, A_{1}, A_{1}\right)=162, N_{E_{7}}\left(A_{1}^{3} * A_{2}, A_{1}, A_{1}\right)=54$, $N_{E_{7}}\left(A_{1}^{5}, A_{1}, A_{1}\right)=0, N_{E_{7}}\left(D_{4}, A_{3}\right)=9, N_{E_{7}}\left(A_{4}, A_{3}\right)=54, N_{E_{7}}\left(A_{1} * A_{3}, A_{3}\right)=135$, $N_{E_{7}}\left(A_{2}^{2}, A_{3}\right)=54, \quad N_{E_{7}}\left(A_{1}^{2} * A_{2}, A_{3}\right)=162, \quad N_{E_{7}}\left(A_{1}^{4}, A_{3}\right)=27$, $N_{E_{7}}\left(D_{4}, A_{1} * A_{2}\right)=45, N_{E_{7}}\left(A_{4}, A_{1} * A_{2}\right)=162, N_{E_{7}}\left(A_{1} * A_{3}, A_{1} * A_{2}\right)=243$, $N_{E_{7}}\left(A_{2}^{2}, A_{1} * A_{2}\right)=54, N_{E_{7}}\left(A_{1}^{2} * A_{2}, A_{1} * A_{2}\right)=162, N_{E_{7}}\left(A_{1}^{4}, A_{1} * A_{2}\right)=27$, $N_{E_{7}}\left(D_{4}, A_{1}^{3}\right)=30, \quad N_{E_{7}}\left(A_{4}, A_{1}^{3}\right)=99, \quad N_{E_{7}}\left(A_{1} * A_{3}, A_{1}^{3}\right)=126$, $N_{E_{7}}\left(A_{2}^{2}, A_{1}^{3}\right)=18, N_{E_{7}}\left(A_{1}^{2} * A_{2}, A_{1}^{3}\right)=54, N_{E_{7}}\left(A_{1}^{4}, A_{1}^{3}\right)=9, N_{E_{7}}\left(D_{4}, A_{2}, A_{1}\right)$ $=81, N_{E_{7}}\left(A_{4}, A_{2}, A_{1}\right)=378, N_{E_{7}}\left(A_{1} * A_{3}, A_{2}, A_{1}\right)=783, N_{E_{7}}\left(A_{2}^{2}, A_{2}, A_{1}\right)=$ $270, N_{E_{7}}\left(A_{1}^{2} * A_{2}, A_{2}, A_{1}\right)=810, N_{E_{7}}\left(A_{1}^{4}, A_{2}, A_{1}\right)=135, N_{E_{7}}\left(D_{4}, A_{1}^{2}, A_{1}\right)=$ 243, $N_{E_{7}}\left(A_{4}, A_{1}^{2}, A_{1}\right)=891, N_{E_{7}}\left(A_{1} * A_{3}, A_{1}^{2}, A_{1}\right)=1377, N_{E_{7}}\left(A_{2}^{2}, A_{1}^{2}, A_{1}\right)=$ $324, N_{E_{7}}\left(A_{1}^{2} * A_{2}, A_{1}^{2}, A_{1}\right)=972, N_{E_{7}}\left(A_{1}^{4}, A_{1}^{2}, A_{1}\right)=162, N_{E_{7}}\left(D_{4}, A_{1}, A_{1}, A_{1}\right)$ $=729, \quad N_{E_{7}}\left(A_{4}, A_{1}, A_{1}, A_{1}\right)=2916, \quad N_{E_{7}}\left(A_{1} * A_{3}, A_{1}, A_{1}, A_{1}\right)=5103$, $N_{E_{7}}\left(A_{2}^{2}, A_{1}, A_{1}, A_{1}\right)=1458, N_{E_{7}}\left(A_{1}^{2} * A_{2}, A_{1}, A_{1}, A_{1}\right)=4374, N_{E_{7}}\left(A_{1}^{4}, A_{1}, A_{1}, A_{1}\right)$ $=729, N_{E_{7}}\left(A_{3}, A_{3}, A_{1}\right)=486, N_{E_{7}}\left(A_{3}, A_{1} * A_{2}, A_{1}\right)=1458, N_{E_{7}}\left(A_{3}, A_{1}^{3}, A_{1}\right)=$ $891, N_{E_{7}}\left(A_{1} * A_{2}, A_{1} * A_{2}, A_{1}\right)=2430, N_{E_{7}}\left(A_{1} * A_{2}, A_{1}^{3}, A_{1}\right)=1215, N_{E_{7}}\left(A_{1}^{3}, A_{1}^{3}, A_{1}\right)$ $=540, N_{E_{7}}\left(A_{3}, A_{2}, A_{2}\right)=432, N_{E_{7}}\left(A_{1} * A_{2}, A_{2}, A_{2}\right)=1188, N_{E_{7}}\left(A_{1}^{3}, A_{2}, A_{2}\right)=$ $711, N_{E_{7}}\left(A_{3}, A_{2}, A_{1}^{2}\right)=1053, N_{E_{7}}\left(A_{1} * A_{2}, A_{2}, A_{1}^{2}\right)=2349, N_{E_{7}}\left(A_{1}^{3}, A_{2}, A_{1}^{2}\right)=$ $1323, N_{E_{7}}\left(A_{3}, A_{1}^{2}, A_{1}^{2}\right)=2430, N_{E_{7}}\left(A_{1} * A_{2}, A_{1}^{2}, A_{1}^{2}\right)=3402, N_{E_{7}}\left(A_{1}^{3}, A_{1}^{2}, A_{1}^{2}\right)=$ $1539, \quad N_{E_{7}}\left(A_{3}, A_{2}, A_{1}, A_{1}\right)=3402, \quad N_{E_{7}}\left(A_{1} * A_{2}, A_{2}, A_{1}, A_{1}\right)=8262$, $N_{E_{7}}\left(A_{1}^{3}, A_{2}, A_{1}, A_{1}\right)=4779, N_{E_{7}}\left(A_{3}, A_{1}^{2}, A_{1}, A_{1}\right)=8019, N_{E_{7}}\left(A_{1} * A_{2}, A_{1}^{2}, A_{1}, A_{1}\right)$ $=13851, N_{E_{7}}\left(A_{1}^{3}, A_{1}^{2}, A_{1}, A_{1}\right)=7047, N_{E_{7}}\left(A_{3}, A_{1}, A_{1}, A_{1}, A_{1}\right)=26244, N_{E_{7}}\left(A_{1} *\right.$ $\left.A_{2}, A_{1}, A_{1}, A_{1}, A_{1}\right)=52488, N_{E_{7}}\left(A_{1}^{3}, A_{1}, A_{1}, A_{1}, A_{1}\right)=28431, N_{E_{7}}\left(A_{2}, A_{2}, A_{2}, A_{1}\right)$ $=2916, \quad N_{E_{7}}\left(A_{2}, A_{2}, A_{1}^{2}, A_{1}\right)=6561, \quad N_{E_{7}}\left(A_{2}, A_{1}^{2}, A_{1}^{2}, A_{1}\right)=13122$, $N_{E_{7}}\left(A_{1}^{2}, A_{1}^{2}, A_{1}^{2}, A_{1}\right)=19683, \quad N_{E_{7}}\left(A_{2}, A_{2}, A_{1}, A_{1}, A_{1}\right)=21870$, $N_{E_{7}}\left(A_{2}, A_{1}^{2}, A_{1}, A_{1}, A_{1}\right)=45927, \quad N_{E_{7}}\left(A_{1}^{2}, A_{1}^{2}, A_{1}, A_{1}, A_{1}\right)=78732$, $N_{E_{7}}\left(A_{2}, A_{1}, A_{1}, A_{1}, A_{1}, A_{1}\right)=157464, \quad N_{E_{7}}\left(A_{1}^{2}, A_{1}, A_{1}, A_{1}, A_{1}, A_{1}\right)=295245$, 
$N_{E_{7}}\left(A_{1}, A_{1}, A_{1}, A_{1}, A_{1}, A_{1}, A_{1}\right)=1062882$, plus the assignments implied by (2.2) and (2.3), with all other numbers $N_{E_{7}}\left(T_{1}, T_{2}, \ldots, T_{d}\right)$ being zero.

A.7. The decomposition numbers for type $E_{8}$ [28, Sec. 7]. We have $N_{E_{8}}\left(E_{8}\right)$ $=1, N_{E_{8}}\left(E_{7}, A_{1}\right)=15, N_{E_{8}}\left(D_{7}, A_{1}\right)=15, N_{E_{8}}\left(A_{7}, A_{1}\right)=15, N_{E_{8}}\left(A_{1} * E_{6}, A_{1}\right)$ $=15, N_{E_{8}}\left(A_{1} * D_{6}, A_{1}\right)=0, N_{E_{8}}\left(A_{1} * A_{6}, A_{1}\right)=15, N_{E_{8}}\left(A_{2} * D_{5}, A_{1}\right)=15$, $N_{E_{8}}\left(A_{2} * A_{5}, A_{1}\right)=0, \quad N_{E_{8}}\left(A_{1}^{2} * D_{5}, A_{1}\right)=0, \quad N_{E_{8}}\left(A_{1}^{2} * A_{5}, A_{1}\right)=0$, $N_{E_{8}}\left(A_{3} * D_{4}, A_{1}\right)=0, N_{E_{8}}\left(A_{3} * A_{4}, A_{1}\right)=15, N_{E_{8}}\left(A_{1} * A_{2} * D_{4}, A_{1}\right)=0$, $N_{E_{8}}\left(A_{1} * A_{2} * A_{4}, A_{1}\right)=15, N_{E_{8}}\left(A_{1}^{3} * D_{4}, A_{1}\right)=0, N_{E_{8}}\left(A_{1}^{3} * A_{4}, A_{1}\right)=0$, $N_{E_{8}}\left(A_{1} * A_{3}^{2}, A_{1}\right)=0, N_{E_{8}}\left(A_{2}^{2} * A_{3}, A_{1}\right)=0, N_{E_{8}}\left(A_{1}^{2} * A_{2} * A_{3}, A_{1}\right)=0$, $N_{E_{8}}\left(A_{1}^{4} * A_{3}, A_{1}\right)=0, \quad N_{E_{8}}\left(A_{1} * A_{2}^{3}, A_{1}\right)=0, \quad N_{E_{8}}\left(A_{1}^{3} * A_{2}^{2}, A_{1}\right)=0$, $N_{E_{8}}\left(A_{1}^{5} * A_{2}, A_{1}\right)=0, N_{E_{8}}\left(A_{1}^{7}, A_{1}\right)=0, N_{E_{8}}\left(E_{6}, A_{2}\right)=20, N_{E_{8}}\left(D_{6}, A_{2}\right)=15$, $N_{E_{8}}\left(A_{6}, A_{2}\right)=60, \quad N_{E_{8}}\left(A_{1} * D_{5}, A_{2}\right)=60, \quad N_{E_{8}}\left(A_{1} * A_{5}, A_{2}\right)=60$, $N_{E_{8}}\left(A_{2} * D_{4}, A_{2}\right)=20, \quad N_{E_{8}}\left(A_{2} * A_{4}, A_{2}\right)=90, \quad N_{E_{8}}\left(A_{3}^{2}, A_{2}\right)=45$, $N_{E_{8}}\left(A_{1}^{2} * D_{4}, A_{2}\right)=0, N_{E_{8}}\left(A_{1}^{2} * A_{4}, A_{2}\right)=90, N_{E_{8}}\left(A_{1} * A_{2} * A_{3}, A_{2}\right)=90$, $N_{E_{8}}\left(A_{1}^{3} * A_{3}, A_{2}\right)=0, N_{E_{8}}\left(A_{2}^{3}, A_{2}\right)=0, N_{E_{8}}\left(A_{1}^{2} * A_{2}^{2}, A_{2}\right)=45, N_{E_{8}}\left(A_{1}^{4} * A_{2}, A_{2}\right)=$ $0, N_{E_{8}}\left(A_{1}^{6}, A_{2}\right)=0, N_{E_{8}}\left(E_{6}, A_{1}^{2}\right)=45, N_{E_{8}}\left(D_{6}, A_{1}^{2}\right)=90, N_{E_{8}}\left(A_{6}, A_{1}^{2}\right)=135$, $N_{E_{8}}\left(A_{1} * D_{5}, A_{1}^{2}\right)=135, N_{E_{8}}\left(A_{1} * A_{5}, A_{1}^{2}\right)=135, N_{E_{8}}\left(A_{2} * D_{4}, A_{1}^{2}\right)=45, N_{E_{8}}\left(A_{2} *\right.$ $\left.A_{4}, A_{1}^{2}\right)=90, N_{E_{8}}\left(A_{3}^{2}, A_{1}^{2}\right)=45, N_{E_{8}}\left(A_{1}^{2} * D_{4}, A_{1}^{2}\right)=0, N_{E_{8}}\left(A_{1}^{2} * A_{4}, A_{1}^{2}\right)=90$, $N_{E_{8}}\left(A_{1} * A_{2} * A_{3}, A_{1}^{2}\right)=90, N_{E_{8}}\left(A_{1}^{3} * A_{3}, A_{1}^{2}\right)=0, N_{E_{8}}\left(A_{2}^{3}, A_{1}^{2}\right)=0, N_{E_{8}}\left(A_{1}^{2} *\right.$ $\left.A_{2}^{2}, A_{1}^{2}\right)=45, N_{E_{8}}\left(A_{1}^{4} * A_{2}, A_{1}^{2}\right)=0, N_{E_{8}}\left(A_{1}^{6}, A_{1}^{2}\right)=0, N_{E_{8}}\left(E_{6}, A_{1}, A_{1}\right)=150$, $N_{E_{8}}\left(D_{6}, A_{1}, A_{1}\right)=225, N_{E_{8}}\left(A_{6}, A_{1}, A_{1}\right)=450, N_{E_{8}}\left(A_{1} * D_{5}, A_{1}, A_{1}\right)=450$, $N_{E_{8}}\left(A_{1} * A_{5}, A_{1}, A_{1}\right)=450, N_{E_{8}}\left(A_{2} * D_{4}, A_{1}, A_{1}\right)=150, N_{E_{8}}\left(A_{2} * A_{4}, A_{1}, A_{1}\right)=$ $450, N_{E_{8}}\left(A_{3}^{2}, A_{1}, A_{1}\right)=225, N_{E_{8}}\left(A_{1}^{2} * D_{4}, A_{1}, A_{1}\right)=0, N_{E_{8}}\left(A_{1}^{2} * A_{4}, A_{1}, A_{1}\right)=450$, $N_{E_{8}}\left(A_{1} * A_{2} * A_{3}, A_{1}, A_{1}\right)=450, N_{E_{8}}\left(A_{1}^{3} * A_{3}, A_{1}, A_{1}\right)=0, N_{E_{8}}\left(A_{2}^{3}, A_{1}, A_{1}\right)=$ $0, N_{E_{8}}\left(A_{1}^{2} * A_{2}^{2}, A_{1}, A_{1}\right)=225, N_{E_{8}}\left(A_{1}^{4} * A_{2}, A_{1}, A_{1}\right)=0, N_{E_{8}}\left(A_{1}^{6}, A_{1}, A_{1}\right)=$ $0, N_{E_{8}}\left(D_{5}, A_{3}\right)=45, N_{E_{8}}\left(A_{5}, A_{3}\right)=90, N_{E_{8}}\left(A_{1} * A_{4}, A_{3}\right)=315, N_{E_{8}}\left(A_{1} *\right.$ $\left.D_{4}, A_{3}\right)=45, N_{E_{8}}\left(A_{2} * A_{3}, A_{3}\right)=270, N_{E_{8}}\left(A_{1}^{2} * A_{3}, A_{3}\right)=270, N_{E_{8}}\left(A_{1} *\right.$ $\left.A_{2}^{2}, A_{3}\right)=225, N_{E_{8}}\left(A_{1}^{3} * A_{2}, A_{3}\right)=225, N_{E_{8}}\left(A_{1}^{5}, A_{3}\right)=0, N_{E_{8}}\left(D_{5}, A_{1} * A_{2}\right)=195$, $N_{E_{8}}\left(A_{5}, A_{1} * A_{2}\right)=390, N_{E_{8}}\left(A_{1} * A_{4}, A_{1} * A_{2}\right)=690, N_{E_{8}}\left(A_{1} * D_{4}, A_{1} * A_{2}\right)$ $=195, \quad N_{E_{8}}\left(A_{2} * A_{3}, A_{1} * A_{2}\right)=495, \quad N_{E_{8}}\left(A_{1}^{2} * A_{3}, A_{1} * A_{2}\right)=495$, $N_{E_{8}}\left(A_{1} * A_{2}^{2}, A_{1} * A_{2}\right)=300, \quad N_{E_{8}}\left(A_{1}^{3} * A_{2}, A_{1} * A_{2}\right)=300$, $N_{E_{8}}\left(A_{1}^{5}, A_{1} * A_{2}\right)=0, \quad N_{E_{8}}\left(D_{5}, A_{1}^{3}\right)=150, \quad N_{E_{8}}\left(A_{5}, A_{1}^{3}\right)=300$, $N_{E_{8}}\left(A_{1} * A_{4}, A_{1}^{3}\right)=375, N_{E_{8}}\left(A_{1} * D_{4}, A_{1}^{3}\right)=150, N_{E_{8}}\left(A_{2} * A_{3}, A_{1}^{3}\right)=225$, $N_{E_{8}}\left(A_{1}^{2} * A_{3}, A_{1}^{3}\right)=225, N_{E_{8}}\left(A_{1} * A_{2}^{2}, A_{1}^{3}\right)=75, N_{E_{8}}\left(A_{1}^{3} * A_{2}, A_{1}^{3}\right)=75$, $N_{E_{8}}\left(A_{1}^{5}, A_{1}^{3}\right)=0, \quad N_{E_{8}}\left(D_{5}, A_{2}, A_{1}\right)=375, \quad N_{E_{8}}\left(A_{5}, A_{2}, A_{1}\right)=750$, $N_{E_{8}}\left(A_{1} * A_{4}, A_{2}, A_{1}\right)=1950, N_{E_{8}}\left(A_{1} * D_{4}, A_{2}, A_{1}\right)=375, N_{E_{8}}\left(A_{2} * A_{3}, A_{2}, A_{1}\right)$ $=1575, \quad N_{E_{8}}\left(A_{1}^{2} * A_{3}, A_{2}, A_{1}\right)=1575, \quad N_{E_{8}}\left(A_{1} * A_{2}^{2}, A_{2}, A_{1}\right)=1200$, $N_{E_{8}}\left(A_{1}^{3} * A_{2}, A_{2}, A_{1}\right)=1200, N_{E_{8}}\left(A_{1}^{5}, A_{2}, A_{1}\right)=0, N_{E_{8}}\left(D_{5}, A_{1}^{2}, A_{1}\right)=1125$, $N_{E_{8}}\left(A_{5}, A_{1}^{2}, A_{1}\right)=2250, \quad N_{E_{8}}\left(A_{1} * A_{4}, A_{1}^{2}, A_{1}\right)=3825, N_{E_{8}}\left(A_{1} * D_{4}, A_{1}^{2}, A_{1}\right)$ $=1125, \quad N_{E_{8}}\left(A_{2} * A_{3}, A_{1}^{2}, A_{1}\right)=2700, N_{E_{8}}\left(A_{1}^{2} * A_{3}, A_{1}^{2}, A_{1}\right)=2700$, $N_{E_{8}}\left(A_{1} * A_{2}^{2}, A_{1}^{2}, A_{1}\right)=1575, N_{E_{8}}\left(A_{1}^{3} * A_{2}, A_{1}^{2}, A_{1}\right)=1575, N_{E_{8}}\left(A_{1}^{5}, A_{1}^{2}, A_{1}\right)=0$, $N_{E_{8}}\left(D_{5}, A_{1}, A_{1}, A_{1}\right)=3375, N_{E_{8}}\left(A_{5}, A_{1}, A_{1}, A_{1}\right)=6750, N_{E_{8}}\left(A_{1} * A_{4}, A_{1}, A_{1}, A_{1}\right)$ $=13500, N_{E_{8}}\left(A_{1} * D_{4}, A_{1}, A_{1}, A_{1}\right)=3375, N_{E_{8}}\left(A_{2} * A_{3}, A_{1}, A_{1}, A_{1}\right)=10125$, $N_{E_{8}}\left(A_{1}^{2} * A_{3}, A_{1}, A_{1}, A_{1}\right)=10125, \quad N_{E_{8}}\left(A_{1} * A_{2}^{2}, A_{1}, A_{1}, A_{1}\right)=6750$, $N_{E_{8}}\left(A_{1}^{3} * A_{2}, A_{1}, A_{1}, A_{1}\right)=6750, N_{E_{8}}\left(A_{1}^{5}, A_{1}, A_{1}, A_{1}\right)=0, N_{E_{8}}\left(D_{4}, D_{4}\right)=5$, $N_{E_{8}}\left(D_{4}, A_{4}\right)=15, N_{E_{8}}\left(A_{4}, A_{4}\right)=138, N_{E_{8}}\left(D_{4}, A_{1} * A_{3}\right)=105, N_{E_{8}}\left(A_{4}, A_{1} * A_{3}\right)=$ $390, N_{E_{8}}\left(A_{1} * A_{3}, A_{1} * A_{3}\right)=1155, N_{E_{8}}\left(D_{4}, A_{2}^{2}\right)=35, N_{E_{8}}\left(A_{4}, A_{2}^{2}\right)=180, N_{E_{8}}\left(A_{1} *\right.$ $\left.A_{3}, A_{2}^{2}\right)=360, \quad N_{E_{8}}\left(A_{2}^{2}, A_{2}^{2}\right)=95, \quad N_{E_{8}}\left(D_{4}, A_{1}^{2} * A_{2}\right)=135$, 
$N_{E_{8}}\left(A_{4}, A_{1}^{2} * A_{2}\right)=630, N_{E_{8}}\left(A_{1} * A_{3}, A_{1}^{2} * A_{2}\right)=1035, N_{E_{8}}\left(A_{2}^{2}, A_{1}^{2} * A_{2}\right)=$ $270, N_{E_{8}}\left(A_{1}^{2} * A_{2}, A_{1}^{2} * A_{2}\right)=495, N_{E_{8}}\left(D_{4}, A_{1}^{4}\right)=30, N_{E_{8}}\left(A_{4}, A_{1}^{4}\right)=165$, $N_{E_{8}}\left(A_{1} * A_{3}, A_{1}^{4}\right)=255, N_{E_{8}}\left(A_{2}^{2}, A_{1}^{4}\right)=60, N_{E_{8}}\left(A_{1}^{2} * A_{2}, A_{1}^{4}\right)=135, N_{E_{8}}\left(A_{1}^{4}, A_{1}^{4}\right)$ $=30, N_{E_{8}}\left(D_{4}, A_{3}, A_{1}\right)=225, N_{E_{8}}\left(A_{4}, A_{3}, A_{1}\right)=1215, N_{E_{8}}\left(A_{1} * A_{3}, A_{3}, A_{1}\right)$ $=4050, N_{E_{8}}\left(A_{2}^{2}, A_{3}, A_{1}\right)=1575, N_{E_{8}}\left(A_{1}^{2} * A_{2}, A_{3}, A_{1}\right)=5400, N_{E_{8}}\left(A_{1}^{4}, A_{3}, A_{1}\right)$ $=1350, \quad N_{E_{8}}\left(D_{4}, A_{1} * A_{2}, A_{1}\right)=975, \quad N_{E_{8}}\left(A_{4}, A_{1} * A_{2}, A_{1}\right)=4590$, $N_{E_{8}}\left(A_{1} * A_{3}, A_{1} * A_{2}, A_{1}\right)=10800, \quad N_{E_{8}}\left(A_{2}^{2}, A_{1} * A_{2}, A_{1}\right)=3450$, $N_{E_{8}}\left(A_{1}^{2} * A_{2}, A_{1} * A_{2}, A_{1}\right)=9900, N_{E_{8}}\left(A_{1}^{4}, A_{1} * A_{2}, A_{1}\right)=2475, N_{E_{8}}\left(D_{4}, A_{1}^{3}, A_{1}\right)$ $=750, N_{E_{8}}\left(A_{4}, A_{1}^{3}, A_{1}\right)=3375, N_{E_{8}}\left(A_{1} * A_{3}, A_{1}^{3}, A_{1}\right)=6750, N_{E_{8}}\left(A_{2}^{2}, A_{1}^{3}, A_{1}\right)$ $=1875, N_{E_{8}}\left(A_{1}^{2} * A_{2}, A_{1}^{3}, A_{1}\right)=4500, N_{E_{8}}\left(A_{1}^{4}, A_{1}^{3}, A_{1}\right)=1125, N_{E_{8}}\left(D_{4}, A_{2}, A_{2}\right)$ $=175, N_{E_{8}}\left(A_{4}, A_{2}, A_{2}\right)=1140, N_{E_{8}}\left(A_{1} * A_{3}, A_{2}, A_{2}\right)=3300, N_{E_{8}}\left(A_{2}^{2}, A_{2}, A_{2}\right)$ $=1300, N_{E_{8}}\left(A_{1}^{2} * A_{2}, A_{2}, A_{2}\right)=4500, N_{E_{8}}\left(A_{1}^{4}, A_{2}, A_{2}\right)=1125, N_{E_{8}}\left(D_{4}, A_{2}, A_{1}^{2}\right)$ $=675, N_{E_{8}}\left(A_{4}, A_{2}, A_{1}^{2}\right)=3015, N_{E_{8}}\left(A_{1} * A_{3}, A_{2}, A_{1}^{2}\right)=8550, N_{E_{8}}\left(A_{2}^{2}, A_{2}, A_{1}^{2}\right)$ $=2925, N_{E_{8}}\left(A_{1}^{2} * A_{2}, A_{2}, A_{1}^{2}\right)=9000, N_{E_{8}}\left(A_{1}^{4}, A_{2}, A_{1}^{2}\right)=2250, N_{E_{8}}\left(D_{4}, A_{1}^{2}, A_{1}^{2}\right)$ $=1800, N_{E_{8}}\left(A_{4}, A_{1}^{2}, A_{1}^{2}\right)=8640, N_{E_{8}}\left(A_{1} * A_{3}, A_{1}^{2}, A_{1}^{2}\right)=17550, N_{E_{8}}\left(A_{2}^{2}, A_{1}^{2}, A_{1}^{2}\right)$ $=5175, \quad N_{E_{8}}\left(A_{1}^{2} * A_{2}, A_{1}^{2}, A_{1}^{2}\right)=13500, N_{E_{8}}\left(A_{1}^{4}, A_{1}^{2}, A_{1}^{2}\right)=3375$, $N_{E_{8}}\left(D_{4}, A_{2}, A_{1}, A_{1}\right)=1875, N_{E_{8}}\left(A_{4}, A_{2}, A_{1}, A_{1}\right)=9450, N_{E_{8}}\left(A_{1} * A_{3}, A_{2}, A_{1}, A_{1}\right)$ $=27000, \quad N_{E_{8}}\left(A_{2}^{2}, A_{2}, A_{1}, A_{1}\right)=9750, \quad N_{E_{8}}\left(A_{1}^{2} * A_{2}, A_{2}, A_{1}, A_{1}\right)=31500$, $N_{E_{8}}\left(A_{1}^{4}, A_{2}, A_{1}, A_{1}\right)=7875, N_{E_{8}}\left(D_{4}, A_{1}^{2}, A_{1}, A_{1}\right)=5625, N_{E_{8}}\left(A_{4}, A_{1}^{2}, A_{1}, A_{1}\right)=$ $26325, \quad N_{E_{8}}\left(A_{1} * A_{3}, A_{1}^{2}, A_{1}, A_{1}\right)=60750, \quad N_{E_{8}}\left(A_{2}^{2}, A_{1}^{2}, A_{1}, A_{1}\right)=19125$, $N_{E_{8}}\left(A_{1}^{2} * A_{2}, A_{1}^{2}, A_{1}, A_{1}\right)=54000, \quad N_{E_{8}}\left(A_{1}^{4}, A_{1}^{2}, A_{1}, A_{1}\right)=13500$, $N_{E_{8}}\left(D_{4}, A_{1}, A_{1}, A_{1}, A_{1}\right)=16875, \quad N_{E_{8}}\left(A_{4}, A_{1}, A_{1}, A_{1}, A_{1}\right)=81000$, $N_{E_{8}}\left(A_{1} * A_{3}, A_{1}, A_{1}, A_{1}, A_{1}\right)=202500, \quad N_{E_{8}}\left(A_{2}^{2}, A_{1}, A_{1}, A_{1}, A_{1}\right)=67500$, $N_{E_{8}}\left(A_{1}^{2} * A_{2}, A_{1}, A_{1}, A_{1}, A_{1}\right)=202500, \quad N_{E_{8}}\left(A_{1}^{4}, A_{1}, A_{1}, A_{1}, A_{1}\right)=50625$, $N_{E_{8}}\left(A_{3}, A_{3}, A_{2}\right)=1350, N_{E_{8}}\left(A_{3}, A_{1} * A_{2}, A_{2}\right)=5175, N_{E_{8}}\left(A_{3}, A_{1}^{3}, A_{2}\right)=3825$, $N_{E_{8}}\left(A_{1} * A_{2}, A_{1} * A_{2}, A_{2}\right)=15000, \quad N_{E_{8}}\left(A_{1} * A_{2}, A_{1}^{3}, A_{2}\right)=9825$, $N_{E_{8}}\left(A_{1}^{3}, A_{1}^{3}, A_{2}\right)=6000, N_{E_{8}}\left(A_{3}, A_{3}, A_{1}^{2}\right)=4050, N_{E_{8}}\left(A_{3}, A_{1} * A_{2}, A_{1}^{2}\right)=13500$, $N_{E_{8}}\left(A_{3}, A_{1}^{3}, A_{1}^{2}\right)=9450, N_{E_{8}}\left(A_{1} * A_{2}, A_{1} * A_{2}, A_{1}^{2}\right)=30825, N_{E_{8}}\left(A_{1} * A_{2}, A_{1}^{3}, A_{1}^{2}\right)$ $=17325, \quad N_{E_{8}}\left(A_{1}^{3}, A_{1}^{3}, A_{1}^{2}\right)=7875, \quad N_{E_{8}}\left(A_{3}, A_{3}, A_{1}, A_{1}\right)=12150$, $N_{E_{8}}\left(A_{3}, A_{1} * A_{2}, A_{1}, A_{1}\right)=42525, \quad N_{E_{8}}\left(A_{3}, A_{1}^{3}, A_{1}, A_{1}\right)=30375$, $N_{E_{8}}\left(A_{1} * A_{2}, A_{1} * A_{2}, A_{1}, A_{1}\right)=106650, N_{E_{8}}\left(A_{1} * A_{2}, A_{1}^{3}, A_{1}, A_{1}\right)=64125$, $N_{E_{8}}\left(A_{1}^{3}, A_{1}^{3}, A_{1}, A_{1}\right)=33750, N_{E_{8}}\left(A_{3}, A_{2}, A_{2}, A_{1}\right)=10575, N_{E_{8}}\left(A_{3}, A_{2}, A_{1}^{2}, A_{1}\right)$ $=29700, \quad N_{E_{8}}\left(A_{3}, A_{1}^{2}, A_{1}^{2}, A_{1}\right)=76950, N_{E_{8}}\left(A_{1} * A_{2}, A_{2}, A_{2}, A_{1}\right)=35700$, $N_{E_{8}}\left(A_{1} * A_{2}, A_{2}, A_{1}^{2}, A_{1}\right)=84825, \quad N_{E_{8}}\left(A_{1} * A_{2}, A_{1}^{2}, A_{1}^{2}, A_{1}\right)=171450$, $N_{E_{8}}\left(A_{1}^{3}, A_{2}, A_{2}, A_{1}\right)=25125, N_{E_{8}}\left(A_{1}^{3}, A_{2}, A_{1}^{2}, A_{1}\right)=55125, N_{E_{8}}\left(A_{1}^{3}, A_{1}^{2}, A_{1}^{2}, A_{1}\right)$ $=94500, N_{E_{8}}\left(A_{3}, A_{2}, A_{1}, A_{1}, A_{1}\right)=91125, N_{E_{8}}\left(A_{3}, A_{1}^{2}, A_{1}, A_{1}, A_{1}\right)=243000$, $N_{E_{8}}\left(A_{1} * A_{2}, A_{2}, A_{1}, A_{1}, A_{1}\right)=276750, N_{E_{8}}\left(A_{1} * A_{2}, A_{1}^{2}, A_{1}, A_{1}, A_{1}\right)=597375$, $N_{E_{8}}\left(A_{1}^{3}, A_{2}, A_{1}, A_{1}, A_{1}\right)=185625, \quad N_{E_{8}}\left(A_{1}^{3}, A_{1}^{2}, A_{1}, A_{1}, A_{1}\right)=354375$, $N_{E_{8}}\left(A_{3}, A_{1}, A_{1}, A_{1}, A_{1}, A_{1}\right)=759375, N_{E_{8}}\left(A_{1} * A_{2}, A_{1}, A_{1}, A_{1}, A_{1}, A_{1}\right)=2025000$, $N_{E_{8}}\left(A_{1}^{3}, A_{1}, A_{1}, A_{1}, A_{1}, A_{1}\right)=1265625, \quad N_{E_{8}}\left(A_{2}, A_{2}, A_{2}, A_{2}\right)=9350$, $N_{E_{8}}\left(A_{2}, A_{2}, A_{2}, A_{1}^{2}\right)=24975, N_{E_{8}}\left(A_{2}, A_{2}, A_{1}^{2}, A_{1}^{2}\right)=64350, N_{E_{8}}\left(A_{2}, A_{1}^{2}, A_{1}^{2}, A_{1}^{2}\right)$ $=143100, N_{E_{8}}\left(A_{1}^{2}, A_{1}^{2}, A_{1}^{2}, A_{1}^{2}\right)=261225, N_{E_{8}}\left(A_{2}, A_{2}, A_{2}, A_{1}, A_{1}\right)=78000$, $N_{E_{8}}\left(A_{2}, A_{2}, A_{1}^{2}, A_{1}, A_{1}\right)=203625, \quad N_{E_{8}}\left(A_{2}, A_{1}^{2}, A_{1}^{2}, A_{1}, A_{1}\right)=479250$, $N_{E_{8}}\left(A_{1}^{2}, A_{1}^{2}, A_{1}^{2}, A_{1}, A_{1}\right)=951750, \quad N_{E_{8}}\left(A_{2}, A_{2}, A_{1}, A_{1}, A_{1}, A_{1}\right)=641250$, $N_{E_{8}}\left(A_{2}, A_{1}^{2}, A_{1}, A_{1}, A_{1}, A_{1}\right)=1569375, N_{E_{8}}\left(A_{1}^{2}, A_{1}^{2}, A_{1}, A_{1}, A_{1}, A_{1}\right)=3341250$, $N_{E_{8}}\left(A_{2}, A_{1}, A_{1}, A_{1}, A_{1}, A_{1}, A_{1}\right)=5062500, \quad N_{E_{8}}\left(A_{1}^{2}, A_{1}, A_{1}, A_{1}, A_{1}, A_{1}, A_{1}\right)$ $=11390625, N_{E_{8}}\left(A_{1}, A_{1}, A_{1}, A_{1}, A_{1}, A_{1}, A_{1}, A_{1}\right)=37968750$, plus the assignments implied by (2.2) and (2.3), with all other numbers $N_{E_{8}}\left(T_{1}, T_{2}, \ldots, T_{d}\right)$ being zero. 
Note added in proof. Jang Soo Kim succeeded in finding bijective proofs for Corollaries 12, 14, 16-19 in "Chain enumeration of $k$-divisible noncrossing partitions of classical types" (arXiv:0908.2641).

\section{ACKNOWLEDGEMENTS}

The authors thank the anonymous referee for a very careful reading of the original manuscript.

\section{REFERENCES}

[1] D. Armstrong, Generalized noncrossing partitions and combinatorics of Coxeter groups, Mem. Amer. Math. Soc., vol. 202, no. 949, Amer. Math. Soc., Providence, RI, 2009.

[2] C. A. Athanasiadis, On noncrossing and nonnesting partitions for classical reflection groups, Electron. J. Combin. 5 (1998), Article \#R42, 16 pp. MR.1644234 (99i:05204)

[3] C. A. Athanasiadis, On some enumerative aspects of generalized associahedra, European J. Combin. 28 (2007), 1208-1215. MR2305586 (2008c:05005)

[4] C. A. Athanasiadis, T. Brady and C. Watt, Shellability of noncrossing partition lattices, Proc. Amer. Math. Soc. 135 (2007), 939-949. MR2262893(2007j:05221)

[5] C. A. Athanasiadis and V. Reiner, Noncrossing partitions for the group $D_{n}$, SIAM J. Discrete Math. 18 (2004), 397-417. MR2112514 (2006b:06004)

[6] C. A. Athanasiadis and E. Tzanaki, On the enumeration of positive cells in generalized cluster complexes and Catalan hyperplane arrangements, J. Algebraic Combin. 23 (2006), 355-375. MR2236611 (2007c:20095)

[7] C. A. Athanasiadis and E. Tzanaki, Shellability and higher Cohen-Macaulay connectivity of generalized cluster complexes, Israel J. Math. 167 (2008), 177-191. MR.2448023

[8] D. Bessis, The dual braid monoid, Ann. Sci. École Norm. Sup. (4) 36 (2003), 647-683. MR2032983 (2004m:20071)

[9] D. Bessis and R. Corran, Non-crossing partitions of type (e,e,r), Adv. Math. 202 (2006), 1-49. MR2218819(2007a:20035)

[10] P. Biane, Some properties of crossings and partitions, Discrete Math. 175 (1997), 41-53. MR 1475837 (98h:05020)

[11] A. Björner and F. Brenti, Combinatorics of Coxeter groups, Springer-Verlag, New York, 2005. MR2133266 (2006d:05001)

[12] M. Bóna, M. Bousquet, G. Labelle and P. Leroux, Enumeration of m-ary cacti, Adv. Appl. Math. 24 (2000), 22-56. MR.1741339 (2001c:05072)

[13] M. Bousquet, C. Chauve and G. Schaeffer, Énumération et génération aléatoire de cactus $m$-aires, Proceedings of the Colloque LaCIM 2000 (Montréal), P. Leroux (ed.), Publications du LaCIM, vol. 27, 2000, pp. 89-100.

[14] T. Brady, A partial order on the symmetric group and new $K(\pi, 1)$ 's for the braid groups, Adv. Math. 161 (2001), 20-40. MR.1857934(2002k:20066)

[15] T. Brady and C. Watt, $K(\pi, 1)$ 's for Artin groups of finite type, Geom. Dedicata 94 (2002), 225-250. MR1950880 (2004i:20066)

[16] T. Brady and C. Watt, Non-crossing partition lattices in finite reflection groups, Trans. Amer. Math. Soc. 360 (2008), 1983-2005. MR2366971 (2008k:20088)

[17] F. Chapoton, Enumerative properties of generalized associahedra, Séminaire Lotharingien Combin. 51 (2004), Article B51b, 16 pp. MR2080386 (2005e:17013)

[18] P. Edelman, Chain enumeration and noncrossing partitions, Discrete Math. 31 (1981), 171180. MR $583216(81 \mathrm{i}: 05018)$

[19] S. Fomin and N. Reading, Generalized cluster complexes and Coxeter combinatorics, Int. Math. Res. Notices 44 (2005), 2709-2757. MR2181310 (2006g:05230)

[20] S. Fomin and N. Reading, Root systems and generalized associahedra, Geometric Combinatorics, 63-131, IAS/Park City Math. Ser., 13, Amer. Math. Soc., Providence, RI, 2007. MR2383126

[21] S. Fomin and A. Zelevinsky, Y-systems and generalized associahedra, Ann. of Math. (2) 158 (2003), 977-1018. MR 2031858 (2004m:17010)

[22] I. J. Good, Generalizations to several variables of Lagrange's expansion, with applications to stochastic processes, Proc. Cambridge Philos. Soc. 56 (1960), 367-380. MR0123021(23:A352) 
[23] I. P. Goulden and D. M. Jackson, The combinatorial relationship between trees, cacti and certain connection coefficients for the symmetric group, Europ. J. Combin. 13 (1992), 357365. MR1181077 (93g:05148)

[24] J. E. Humphreys, Reflection groups and Coxeter groups, Cambridge University Press, Cambridge, 1990. MR1066460(92h:20002)

[25] J. Irving, Combinatorial constructions for transitive factorizations in the symmetric group, Ph.D. thesis, University of Waterloo, 2004.

[26] C. Krattenthaler, Operator methods and Lagrange inversion: A unified approach to Lagrange formulas, Trans. Amer. Math. Soc. 305 (1988), 431-465. MR.924765 (89d:05017)

[27] C. Krattenthaler, The F-triangle of the generalised cluster complex, in: Topics in Discrete Mathematics, dedicated to Jarik Nešetrril on the occasion of his 60th birthday, M. Klazar, J. Kratochvil, M. Loebl, J. Matoušek, R. Thomas and P. Valtr (eds.), Springer-Verlag, Berlin, New York, 2006, pp. 93-126. MR2249265 (2007g:05194)

[28] C. Krattenthaler, The $M$-triangle of generalised non-crossing partitions for the types $E_{7}$ and $E_{8}$, Séminaire Lotharingien Combin. 54 (2006), Article B54l, 34 pages.

[29] C. Krattenthaler, Non-crossing partitions on an annulus, in preparation.

[30] G. Kreweras, Sur les partitions non croisées d'un cycle, Discrete Math. 1 (1972), 333-350. MR0309747(46:8852)

[31] N. Reading, Chains in the noncrossing partition lattice, SIAM J. Discrete Math. 22 (2008), 875-886. MR2424827

[32] V. Reiner, Non-crossing partitions for classical reflection groups, Discrete Math. 177 (1997), 195-222. MR1483446 (99f:06005)

[33] R. P. Stanley, Enumerative Combinatorics, Vol. 1, Wadsworth \& Brooks/Cole, Pacific Grove, California, 1986; reprinted by Cambridge University Press, Cambridge, 1998. MR 1442260 (98a:05001)

[34] R. P. Stanley, Enumerative Combinatorics, vol. 2, Cambridge University Press, Cambridge, 1999. MR1676282 (2000k:05026)

[35] J. R. Stembridge, coxeter, Maple package for working with root systems and finite Coxeter groups; available at http://www.math.lsa.umich.edu/ ${ }^{\sim}$ jrs.

[36] E. Tzanaki, Combinatorics of generalized cluster complexes and hyperplane arrangements, Ph.D. thesis, University of Crete, Iraklio, 2007.

[37] E. Tzanaki, Polygon dissections and some generalizations of cluster complexes, J. Combin. Theory Ser. A 113 (2006), 1189-1198. MR2244140(2007c:05201)

[38] E. Tzanaki, Faces of generalized cluster complexes and noncrossing partitions, SIAM J. Discrete Math. 22 (2008), 15-30. MR2383226

Fakultät für Mathematik, Universität Wien, Nordbergstrasse 15, A-1090 Vienna, AUSTRIA

$U R L:$ http://www.mat.univie.ac.at/ kratt

School of Mathematical Sciences, Queen Mary \& Westfield College, University of London, Mile End Road, London E1 4NS, United Kingdom

$U R L:$ http://www.maths.qmw.ac.uk/ twm/ 